R. Bezrukavnikov, I. Mirković and D. Rumynin

Nagoya Math. J.

Vol. 184 (2006), 1-55

\title{
SINGULAR LOCALIZATION AND INTERTWINING FUNCTORS FOR REDUCTIVE LIE ALGEBRAS IN PRIME CHARACTERISTIC
}

\author{
ROMAN BEZRUKAVNIKOV, IVAN MIRKOVIĆ AND \\ DMITRIY RUMYNIN
}

\author{
To George Lusztig with admiration
}

\begin{abstract}
In $[\mathrm{BMR}]$ we observed that, on the level of derived categories, representations of the Lie algebra of a semisimple algebraic group over a field of finite characteristic with a given (generalized) regular central character can be identified with coherent sheaves on the formal neighborhood of the corresponding (generalized) Springer fiber. In the present paper we treat singular central characters.

The basic step is the Beilinson-Bernstein localization of modules with a fixed (generalized) central character $\lambda$ as sheaves on the partial flag variety corresponding to the singularity of $\lambda$. These sheaves are modules over a sheaf of algebras which is a version of twisted crystalline differential operators. We discuss translation functors and intertwining functors. The latter generate an action of the affine braid group on the derived category of modules with a regular (generalized) central character, which intertwines different localization functors. We also describe the standard duality on Lie algebra modules in terms of $\mathcal{D}$-modules and coherent sheaves.
\end{abstract}

\section{$\S 0$. Introduction}

This is a sequel to $[\mathrm{BMR}]$. In the first chapter we extend the localization construction for modular representations from [BMR] to arbitrary infinitesimal characters. This is used in the second chapter to study the translation functors. We use translation functors to construct intertwining functors, which generate an action of the affine braid group on the derived

Received February 6, 2006.

Revised November 5, 2006.

2000 Mathematics Subject Classification: Primary 17B50; Secondary 16E20, 18 F99.

R.B. was partially supported by NSF grant DMS-0505466 and Sloan Foundation, D.R. by EPSRC and I.M. by NSF grants. 
categories of regular blocks in modular representation categories. An application is given in chapter three, where we describe the duality operation on representations in a regular block in terms of localization.

More precisely, we consider representations of a Lie algebra $\mathfrak{g}$ of a reductive algebraic group $G$ over a field $\mathbb{k}$ of positive characteristic $p$. (Assumptions on $G$ and $p$ are in Section 1.1.3.) The main result of [BMR] was the description of the derived category of representations of $\mathfrak{g}$ with a given (generalized) regular central character, in terms of coherent sheaves.

The center $\mathfrak{Z}$ of the enveloping algebra $U=U(\mathfrak{g})$ contains the "HarishChandra part" $\mathfrak{Z}_{\mathrm{HC}} \stackrel{\text { def }}{=} \mathrm{U}(\mathfrak{g})^{G}$ isomorphic to the Weyl group invariants $S(\mathfrak{h})^{W}$ in the symmetric algebra of the Cartan algebra $\mathfrak{h}$ of $\mathfrak{g}$. The center also has the "Frobenius part" $\mathfrak{Z}_{\mathrm{Fr}} \cong \mathcal{O}\left(\mathfrak{g}^{*(1)}\right)$, the functions on the Frobenius twist of the dual of the Lie algebra. So, a character of the center is given by a compatible pair $(\lambda, \chi)$ of $\lambda \in \mathfrak{h}^{*}$ and $\chi \in \mathfrak{g}^{*}$. For a regular $\lambda$, the derived representation category corresponding to $(\lambda, \chi)$ is identified in $[\mathrm{BMR}]$ with the derived category of coherent sheaves on the formal neighborhood of the (generalized) Springer fiber corresponding to $\chi$. Here we extend this description of the derived category of representations to singular infinitesimal characters $\lambda$. For each character of the Harish-Chandra center $\mathfrak{Z}_{\mathrm{HC}}$ there is $\lambda$ in the corresponding $W$-orbit in $\mathfrak{h}^{*}$ and a partial flag variety $\mathcal{P}=G / P$ such that the singularity of $\lambda$ is of $\mathcal{P}$-type, i.e. the stabilizer $\operatorname{Stab}_{W}(\lambda)$ is the corresponding parabolic Weyl group. If we extend the Harish-Chandra central character to a character $(\lambda, \chi)$ of the full center, the derived category of modules is then identified with the derived category of coherent sheaves on the formal neighborhood of the corresponding parabolic Springer fiber, a subvariety of $\mathcal{P}$ associated to $\chi$ and $\lambda$. In the most interesting case when $\lambda$ is integral, so $\chi$ is nilpotent, the parabolic Springer fiber is the set of parabolic subalgebras of a given type, containing $\chi$.

The basic step is the Beilinson-Bernstein type localization equivalence of derived categories of modules with (generalized) infinitesimal character $\lambda$, and modules over a sheaf of algebras $\widetilde{\mathcal{D}}_{\mathcal{P}}^{\widehat{\lambda}}$ on the partial flag variety $\mathcal{P}$. Here, $\widetilde{\mathcal{D}}_{\mathcal{P}}^{\widehat{\lambda}}$ is a central completion of the sheaf of algebras $\widetilde{\mathcal{D}}_{\mathcal{P}}$; the latter is a version of twisted crystalline differential operators, but for $\mathcal{P} \neq \mathcal{B}$ it is actually larger than the TDO algebras. For example, in the extreme case $P=G, \mathcal{P}=$ pt we have $\widetilde{\mathcal{D}}_{\mathcal{P}}=\mathrm{U}(\mathfrak{g})$ is the enveloping algebra $\mathrm{U}(\mathfrak{g})$. Next, $\widetilde{\mathcal{D}}_{\mathcal{P}}^{\hat{\lambda}}$ turns out to be an Azumaya algebra that splits on the formal 
neighborhood of a parabolic Springer fiber, and this gives an equivalence with coherent sheaves. We also prove a localization theorem for twisted differential operators on $\mathcal{P}$, see 1.10. The categories of modules with a fixed generalized central character is then related to the smaller version of the parabolic Springer fiber, namely, the set of parabolics of a given type containing the given nilpotent in the radical.

The proofs of these results are, to a large extent, parallel to those of $[\mathrm{BMR}]$, though some new ideas are needed. The new aspect is that singular localization provides a better understanding of translation functors on $\mathfrak{g}$ modules. We show that the closely related intertwining functors generate an action of the affine braid group on the derived category of modules with a fixed (generalized) central character. This action intertwines different localization functors.

The action and its applications will be further discussed in a future publication, see also [B]. An application discussed in the present paper is concerned with a description of the usual duality on finite dimensional Lie algebra modules (with a regular central character) in terms of coherent sheaves; see Remark 3.0.12 for comments on motivation for this result.

0.0.1. It is an honor for us to dedicate this paper to George Lusztig. Our intellectual debt to him can not be overestimated. This paper is a part of the project inspired by his conjectures; but also much of the other work by the authors is an exploration of beautiful landscapes whose very existence would probably remain completely unknown if not for the hints found in Lusztig's papers.

0.0.2. The main part of this work was accomplished when R.B. and I.M. were visiting the Hebrew University in Jerusalem, and while one of the authors (I.M.) was visiting Universite de Cergy-Pontoise in Paris. We thank both institutions for the wonderful working atmosphere.

This work is a continuation of [BMR] and is partly a byproduct of communications acknowledged in loc. cit. We use the opportunity to thank these mathematicians again. We are also grateful to Iain Gordon, Victor Protsak and Alexander Samokhin for helpful correspondence, and to the referees for an extraordinarily thorough job.

Notation. Most of the paper considers schemes over an algebraically closed field $\mathbb{k}$ of characteristic $p>0$. For a closed subscheme $\mathfrak{Y}$ of a scheme 
$\mathfrak{X}$ the category of modules on $\mathfrak{X}$ supported on $\mathfrak{Y}$ is introduced in 1.4.1. The inverse image of sheaves is denoted $f^{-1}$ and for $\mathcal{O}$-modules $f^{*}$ (both direct images are denoted $f_{*}$ ). We denote by $\mathcal{T}_{X}$ and $\mathcal{T}_{X}^{*}$ the sheaves of sections of the (co)tangent bundles $T X$ and $T^{*} X$. We denote by $X / G$ the invariant theory quotient of an affine scheme $X$ by a group $G$.

\section{§1. Localization on partial flag varieties}

For any partial flag variety $\mathcal{P}$ we define a sheaf of algebras $\widetilde{\mathcal{D}}_{\mathcal{P}}$ on $\mathcal{P}$. When $\mathcal{P}$ is the full flag variety $\mathcal{B}$ this is the Beilinson-Bernstein deformation of differential operators on $\mathcal{B}$ by twisted differential operators. When $\mathcal{P}=$ pt this is just the enveloping algebra $U(\mathfrak{g})$. There will be three kinds of geometric objects related to $\widetilde{\mathcal{D}}_{\mathcal{P}}$, besides of the base $\mathcal{P}$ these are the classical limit $\widetilde{\mathfrak{g}}_{\mathcal{P}}^{*}$ of $\widetilde{\mathcal{D}}_{\mathcal{P}}$ and the spectrum $\mathcal{Z}\left(\widetilde{\mathcal{D}}_{\mathcal{P}}\right)$ of the center of $\widetilde{\mathcal{D}}_{\mathcal{P}}$. For any sheaf of algebras $\mathcal{A}$ we denote by $Z(\mathcal{A})$ and $\mathcal{Z}(\mathcal{A})$ the center of $\mathcal{A}$ and the spectrum of the center.

For arbitrary $\lambda \in \mathfrak{h}^{*}$ with "singularity precisely $\mathcal{P}$ " (1), we establish some equivalences of derived categories. The final result is that on the level of derived categories $\mathfrak{g}$-modules with a given generalized central character (with Harish-Chandra part $\lambda$ ) are the same as coherent sheaves on $\widetilde{\mathfrak{g}}_{\mathcal{P}}^{*}$ supported on the corresponding parabolic Springer fiber. An intermediate step is a Beilinson-Bernstein type localization of $\mathrm{U}^{\lambda}$-modules to modules for $\mathcal{D}_{\mathcal{P}}^{\lambda} \stackrel{\text { def }}{=} \widetilde{\mathcal{D}}_{\mathcal{P}} \otimes_{\mathcal{O}\left(\mathfrak{h}^{*}\right)} \mathbb{k}_{\lambda}$. (Actually we construct some equivalences for more general $\lambda$.)

\subsection{Crystalline differential operators on flag varieties}

We start by presenting settings and notations. They are slightly more general than in $[\mathrm{BMR}]$, as the group is now reductive and not necessarily semisimple.

\subsubsection{Reductive group $G$}

Let $G$ be a reductive algebraic group over $\mathbb{k}$. Let $B=T \cdot N$ be a Borel subgroup with the unipotent radical $N$ and a Cartan subgroup $T$. Let $H$ be the (abstract) Cartan group of $G$ so that $B$ gives an isomorphism $\iota_{\mathfrak{b}}=(T \cong B \cong(N \cong H)$. Let $\mathfrak{g}, \mathfrak{b}, \mathfrak{t}, \mathfrak{n}, \mathfrak{h}$ be the corresponding Lie algebras. We call elements of $\Lambda=X^{*}(H)$ characters and elements of $\mathfrak{h}^{*}$ weights. The

\footnotetext{
${ }^{1}$ By this we mean that the stabilizer (with respect to the dot action) of $\lambda$ in the Weyl group $W$ of $G$ is precisely the Weyl group $W_{\mathcal{P}}$ of Levi groups of parabolic subgroups in $\mathcal{P}$.
} 
integral weights are differentials of characters $\mathfrak{h}_{\mathbb{F}_{p}}^{*} \stackrel{\text { def }}{=} d \Lambda \cong \Lambda \otimes_{\mathbb{Z}} \mathbb{F}_{p}$. The character lattice $\Lambda$ contains the set of roots $\Delta$ and positive roots $\Delta^{+}$which are chosen as $T$-roots in $\mathfrak{g} / \mathfrak{b}$ via the above "b-identification" $\iota_{\mathfrak{b}}$. Also, $\Lambda$ contains the root lattice $Q$ generated by $\Delta$, the dominant cone $\Lambda^{+} \subseteq \Lambda$ and the semi-group $Q^{+}$generated by $\Delta^{+}$. Let $I \subseteq \Delta^{+}$be the set of simple roots. For a root $\alpha \in \Delta$ let $\check{\alpha} \in \check{\Delta}$ be the corresponding coroot. For $\lambda, \mu \in \Lambda$ we write $\lambda>\mu$ if $\lambda-\mu$ is a sum of positive roots.

Similarly, $\iota_{\mathfrak{b}}$ identifies $N_{G}(T) / T$ with the Weyl group $W \subseteq \operatorname{Aut}(H)$. We have the standard action of $W$ on $\Lambda$ and on $\mathfrak{h}^{*}=\Lambda \otimes \mathbb{k}$ given by $w: \lambda \mapsto w(\lambda)$.

Any character $\nu \in \Lambda$ defines a line bundle $\mathcal{O}_{\mathcal{B}, \nu}=\mathcal{O}_{\nu}$ on the flag variety $\mathcal{B} \cong G / B$, and a standard $G$-module $V_{\nu} \stackrel{\text { def }}{=} \mathrm{H}^{0}\left(\mathcal{B}, \mathcal{O}_{\nu^{+}}\right)$with extremal weight $\nu$, here $\nu^{+}$denotes the dominant $W$-conjugate of $\nu$ (notice that a dominant weight corresponds to a semi-ample line bundle in our normalization). We will also write $\mathcal{O}_{\nu}$ instead of $\pi^{*}\left(\mathcal{O}_{\nu}\right)$ for a scheme $X$ equipped with a map $\pi: X \rightarrow \mathcal{B}$ (e.g. a subscheme of $\tilde{\mathfrak{g}}^{*}$ ).

\subsubsection{Affine Weyl groups and the dot-action}

Along with the standard action we will also use the dot-action, which differs from the standard action by the $\rho$ shift: $w: \lambda \mapsto w \bullet \lambda \stackrel{\text { def }}{=} w(\lambda+\rho)-\rho$, where $\rho$ is the half sum of positive roots.

We will indicate the dot-action by writing $(W, \bullet)$. The following rule will be observed in most cases: we will use the $\bullet$-action on $\mathfrak{h}^{*}$ (except in a few auxiliary calculations), while the action of $W$ on the Frobenius twist $\mathfrak{h}^{*(1)}$ of $\mathfrak{h}^{*}$ is the standard action of $W$.

Let $W_{\text {aff }} \stackrel{\text { def }}{=} W \ltimes Q \subseteq W_{\text {aff }}^{\prime} \stackrel{\text { def }}{=} W \ltimes \Lambda$ be the affine Weyl group and the extended affine Weyl group.

These groups act on $\Lambda$ and on $\Lambda_{\mathbb{R}}=\Lambda \otimes_{\mathbb{Z}} \mathbb{R}$. The group $W_{\text {aff is generated }}$ by reflections in affine hyperplanes $H_{\check{\alpha}, n}$ given by $\langle\check{\alpha}\rangle=$,$n ; here \check{\alpha}$ is a coroot and $n \in \mathbb{Z}$. Thus $W_{\text {aff }}$ is the affine Weyl group of the Langlands dual group in the standard terminology.

We extend the dot-action to $W_{\text {aff }}$ and $W_{\text {aff }}^{\prime}$ so that $\mu \in \Lambda$ acts by the $p \mu$-translation: $w: \lambda \mapsto w \bullet \lambda \stackrel{\text { def }}{=} p w\left(\frac{\lambda+\rho}{p}\right)-\rho$.

The hyperplanes $H_{\check{\alpha}}$ define a stratification of $\Lambda_{\mathbb{R}}$; by a facet we mean a stratum of the stratification. Open facets are called alcoves and codimension one facets are called faces. The set of alcoves is a torsor for $\left(W_{\text {aff }}, \bullet\right)$. So, 
$\left(W_{\text {aff }}, \bullet\right)$-orbits in the set of faces are canonically identified with the faces in the closure of the fundamental alcove $A_{0}$ (the one that contains $(\epsilon-1) \rho$ for small positive $\epsilon$ ).

The group $W_{\text {aff }}$ is a Coxeter group, the Coxeter generators are reflections in the faces of $A_{0}$; we denote the latter set by $I_{\text {aff }}$. Thus $I_{\text {aff }}$ is the set of vertices of the affine Dynkin diagram corresponding to the root system which is dual to the root system of $G$.

\subsubsection{Restrictions on the group $G$ and the characteristic $p$}

$G$ is a connected reductive algebraic group over a closed field $\mathbb{k}$ of finite characteristic $p$. We assume throughout the so-called standard, or JantzenPremet, conditions on $G$ and the prime $p$ :

- (A) The derived subgroup $G^{\prime}$ is simply connected.

- (B) $p$ is a good prime for $G^{(2)}$, and $p$ is odd ${ }^{(3)}$.

- (C) There exists a non-degenerate invariant bilinear form on $\mathfrak{g}^{(4)}$.

If $p$ satisfies these conditions for a reductive group $G$, then it also does so for a Levi subgroup $L \subset G$, see [Ja, 6.5] or [BG, Lemma 3.2] ${ }^{(5)}$. For a semisimple group $G$ these conditions amount to $p$ being very good for $G$, some claims in $[\mathrm{BMR}]$ have been proved under this assumption. For a reductive group $G$ they ensure the standard structural results that we use such as the following

Lemma. ([BG, Lemma 3.2], [Ja, 7.4]) The centralizer in the group $G$ of any semisimple $h \in \mathfrak{g}$ is a Levi subgroup.

Starting from Chapter 2 we use regular integral weights. So, in order for such weights to exist we need the stronger assumption $p \geq h$ where $h$ is the maximum of the Coxeter numbers of simple factors of $G^{\prime}$. For a simple factor $h=\left\langle\rho, \check{\alpha}_{0}\right\rangle+1$ where $\check{\alpha}_{0}$ is the highest coroot. In [BMR] we mostly worked under the assumption $p>h$.

\footnotetext{
${ }^{2}$ This excludes $G^{\prime}$ having simple factors of type $B, C, D, E, F, G$ if $p=2, E, F, G$ if $p=3$ and $E_{8}$ for $p=5$.

${ }^{3}$ This condition follows from the rest unless all simple factors of $G^{\prime}$ are of type $A$. It is not a part of standard assumptions, and is imposed to conform with the assumptions of $[\mathrm{BG}]$. We expect that it is, in fact, redundant.

${ }^{4}$ For semisimple $G$ satisfying (A) and (B) this amounts to $G$ not having simple factors $S L(m p)$, though $G=G L(n)$ satisfies the assumption for any $n$.

${ }^{5}$ Here and below we use $[\mathrm{BG}]$ as a convenient reference for some basic statements about Lie algebras. We have not attempted to identify the original source of the arguments; most of them go back to Springer, Steinberg or Bardsley and Richardson.
} 


\subsubsection{Crystalline differential operators}

The ring of crystalline differential operators $\mathcal{D}_{Y}$ on a smooth variety $Y$ is the sheaf of algebras generated by functions $\mathcal{O}_{Y}$, vector fields $\mathcal{T}_{Y}$ and the standard relations between them. These include the module, commutator and Lie algebroid relations

$$
\begin{aligned}
& f \cdot \partial=f \partial, \partial \cdot f-f \cdot \partial=\partial(f), \partial \cdot \partial^{\prime}-\partial^{\prime} \cdot \partial=\left[\partial, \partial^{\prime}\right], \\
& \partial, \partial^{\prime} \in \mathcal{T}_{Y}, f \in \mathcal{O}_{Y} .
\end{aligned}
$$

Locally $\mathcal{T}_{Y}$ has a frame $\partial_{i}, 1 \leq i \leq n$, and then $\mathcal{D}_{Y}$ has a frame $\partial^{I}=$ $\partial_{1}^{I_{1}} \cdots \partial_{n}^{I_{n}}, I \in \mathbb{Z}_{+}^{n}$. The sheaf $\mathcal{D}_{Y}$ is an Azumaya algebra ${ }^{(6)}$ on the Frobenius twist $T^{*} Y^{(1)}$ of the cotangent bundle [BMR, Theorem 2.2.3] ${ }^{(7)}$. Here, a vector field $\xi$ on $Y$ has $p^{\text {th }}$ power $\xi^{p}$ in $\mathcal{D}_{Y}$ and also another kind of $p^{\text {th }}$ power $\xi^{[p]}$, calculated this time in operators on $\mathcal{O}_{Y}$, which happens to be a vector field again. Now $\mathcal{T}_{Y}{ }^{(1)} \subseteq \mathcal{O}_{T^{*} Y^{(1)}}$ maps to differential operators by $\mathcal{T}_{Y}^{(1)} \ni \xi^{(1)} \mapsto \iota\left(\xi^{(1)}\right) \stackrel{\text { def }}{=} \xi^{p}-\xi^{[p]} \in \mathcal{D}_{Y}$, the difference of two $p^{\text {th }}$ powers. This gives an isomorphism $\iota: \mathcal{O}_{T^{*} Y^{(1)}} \rightarrow Z\left(\mathcal{D}_{Y}\right)$. When $Y$ is a torus $T$ then $\iota$ in particular maps $S \mathfrak{t}^{(1)}$ into $S \mathfrak{t}$, the corresponding map of spaces is the Artin-Schreier map which we denote $\mathrm{AS}=\mathrm{AS}_{T}: \mathfrak{t}^{*} \rightarrow \mathfrak{t}^{*(1)}$. If $\lambda$ is the differential of a character of $T$ and $c \in \mathbb{k}$, then $\operatorname{AS}(c \lambda)=\left(c^{p}-c\right) \lambda^{(1)}$; thus one can identify $\mathfrak{h}^{*}$ with the affine space $\mathbb{A}^{n}$ so that AS is identified with the product of $n$ copies of the standard Artin-Schreier map.

1.1.5. The Harish-Chandra and Frobenius ingredients of the center of $\mathrm{U}(\mathfrak{g})$

Let now $U \stackrel{\text { def }}{=} U(\mathfrak{g})$ be the enveloping algebra of $\mathfrak{g}$. The subalgebra of $G$-invariants $\mathfrak{Z}_{\mathrm{HC}} \stackrel{\text { def }}{=} \mathrm{U}(\mathfrak{g})^{G}$ is central in $\mathrm{U}(\mathfrak{g})$. On the other hand as the algebra of left invariant differential operators, $\mathrm{U}=\mathcal{D}(G)^{G \times 1}$ has a central subalgebra $\mathcal{O}\left(T^{*} G^{(1)}\right)^{G \times 1}=\mathcal{O}\left(\mathfrak{g}^{*(1)}\right)$ which we call the Frobenius center $\mathfrak{Z}_{\mathrm{Fr}}$.

Theorem. ([Ve], [KW], [MR]) (a) There is a canonical isomorphism $\mathrm{U}^{G} \stackrel{i_{\mathrm{HC}}}{\longrightarrow} S(\mathfrak{h})^{(W, \bullet)(8)}$.

${ }^{6}$ An Azumaya algebra is a sheaf of locally matrix algebras in etale topology [Mln].

${ }^{7}$ See also $[\mathrm{OV}]$ for more information.

${ }^{8} \mathrm{~A}$ geometric description of $i_{\mathrm{HC}}$ is sketched in 1.2.2. 
(b) The center $\mathfrak{Z}$ of $\mathrm{U}$ is a combination of the Harish-Chandra part and the Frobenius part

$$
\mathfrak{Z} \cong \mathfrak{Z}_{\mathrm{Fr}} \otimes_{\mathfrak{Z}_{\mathrm{Fr}} \cap \mathfrak{Z}_{\mathrm{HC}}} \mathfrak{Z}_{\mathrm{HC}}, \text { i.e., } \quad \mathcal{Z}(\mathrm{U})=\mathfrak{g}^{*(1)} \times_{\mathfrak{h}^{*(1)} / W} \mathfrak{h}^{*} / W .
$$

Here, $\mathfrak{g}^{*(1)} \rightarrow \mathfrak{h}^{*(1)} / W$ is the adjoint quotient, while the map $\mathfrak{h}^{*} / W \rightarrow$ $\mathfrak{h}^{*(1)} / W$ comes from the Artin-Schreier map $\mathfrak{h}^{*} \stackrel{\text { AS }}{\longrightarrow} \mathfrak{h}^{*(1)}$, cf. Section 1.1.4.

For $\lambda \in \mathfrak{h}^{*}$ and $\chi \in \mathfrak{g}^{*(1)}$, we introduce the central specializations of the universal enveloping algebra: $\mathrm{U}^{\lambda} \stackrel{\text { def }}{=} \mathrm{U} \otimes_{\mathfrak{Z}_{\mathrm{HC}}} \mathbb{k}_{\lambda}, u_{\chi} \stackrel{\text { def }}{=} \mathrm{U} \otimes_{\mathfrak{Z}_{\mathrm{Fr}}} \mathbb{k}_{\chi^{(1)}}$, and $\mathrm{U}_{\chi}^{\lambda} \stackrel{\text { def }}{=} \mathrm{U} \otimes_{\mathfrak{Z}} \mathbb{k}_{(\chi, \lambda)}$. These constructions can be applied to a parabolic subalgebra $\mathfrak{p}$ or its Levi factor $\overline{\mathfrak{p}}$ by restricting the reduction data to the corresponding subalgebras. For instance, $U_{\chi}^{\lambda}(\overline{\mathfrak{p}})=\mathrm{U}(\overline{\mathfrak{p}}) \otimes_{\mathfrak{Z}(\overline{\mathfrak{p}})} \mathbb{k}_{\left(\left.\chi\right|_{\overline{\mathfrak{p}}},\left.\lambda\right|_{\overline{\mathfrak{p}}}\right)}$ or $u_{\chi}(\mathfrak{p})$ is the reduced enveloping algebra. We also introduce the extended universal enveloping algebra $\widetilde{U}=\mathrm{U} \otimes_{\mathfrak{Z}_{\mathrm{HC}}} S(\mathfrak{h})$, denoted $\widetilde{\mathrm{U}}(\overline{\mathfrak{p}})$ when applied to a Levi factor.

\subsubsection{Unramified weights}

We will say that $\lambda \in \mathfrak{h}^{*}$ is unramified if the map $\mathrm{AS} / W: \mathfrak{h}^{*} / W \rightarrow$ $\mathfrak{h}^{*(1)} / W$ is unramified at $W \bullet \lambda$. This is equivalent to the equality of the stabilizers $W_{\lambda} \subseteq W_{\mathrm{AS}(\lambda)}$. Notice that the stabilizers are Coxeter subgroups of $W$, in fact, they are the Weyl groups of the Levi factors in Lemma 1.1.3. It is easy to observe that $\operatorname{AS}(w \lambda)=\operatorname{AS}(w \bullet \lambda)=w \operatorname{AS}(\lambda)$. So, a weight $\lambda$ is ramified if and only if for some root $\alpha$ one has $\langle\check{\alpha}, \lambda+\rho\rangle \in \mathbb{F}_{p}^{*}=$ $\mathbb{F}_{p}-\{0\}$, i.e., $\lambda$ is integral and regular for $\alpha$. It is well known that such ramification produces reducibility of standard $\mathfrak{g}$-modules. In the unramified case the representation theory simplifies, because the corresponding central reductions (or completions) of the enveloping algebra are Azumaya [BG, Theorems 2.6 and 3.10], [BrGo].

Let $\mathfrak{h}_{\text {unr }}^{*} \subset \mathfrak{h}^{*}$ be the open set of all unramified weights.

\subsubsection{Azumaya locus}

The central variety $\mathcal{Z}(\mathrm{U})$ contains an open part

$$
\begin{aligned}
\mathcal{Z}(\mathrm{U})_{\mathrm{unr}} & \stackrel{\text { def }}{=} \mathcal{Z}(\mathrm{U}) \times_{\mathfrak{h}^{*} / W} \mathfrak{h}_{\mathrm{unr}}^{*} / W=\mathfrak{g}^{*(1)} \times_{\mathfrak{h}^{*(1)} / W} \mathfrak{h}_{\mathrm{unr}}^{*} / W \\
& \subseteq \mathfrak{g}^{*(1)} \times_{\mathfrak{h}^{*(1)} / W} \mathfrak{h}^{*} / W=\mathcal{Z}(\mathrm{U}) .
\end{aligned}
$$

$\mathrm{U}$ is known to be generically Azumaya and the Azumaya locus in $\mathcal{Z}(\mathrm{U})$ coincides with the smooth locus (see Theorems 2.5, 2.6 in [BG], or [BrGo] 
for the semi-simple case). The variety $\mathcal{Z}(\mathrm{U})$ unr is smooth, because it admits an etale map to $\mathfrak{g}^{*(1)}$; thus $\mathrm{U}$ is Azumaya over $\mathcal{Z}(\mathrm{U})_{\text {unr }}$.

\subsection{The algebras $\widetilde{\mathcal{D}}_{\mathcal{P}}$ on partial flag varieties $\mathcal{P}$}

In this section we define and discuss various sheaves of rings related to $\mathfrak{g}$; further sections will be devoted to relations between the (derived) categories of sheaves of modules. In 1.2.1 we start with a general construction that in particular produces algebras $\widetilde{\mathcal{D}}_{\mathcal{P}}$. A reader unfamiliar with torsors can consult $[\mathrm{Sk}]$.

\subsubsection{The algebras $\widetilde{\mathcal{D}}_{X}$ associated to torsors}

A torsor $\widetilde{X} \stackrel{\pi}{\rightarrow} X$ for a reductive group $L$ defines a Lie algebroid $\widetilde{\mathcal{T}}_{X} \stackrel{\text { def }}{=} \pi_{*}\left(\mathcal{T}_{\widetilde{X}}\right)^{L}$ with the enveloping algebra $\widetilde{\mathcal{D}}_{X} \stackrel{\text { def }}{=} \pi_{*}\left(\mathcal{D}_{\tilde{X}}\right)^{L}$. Let $\mathfrak{l}$ be the Lie algebra of $L$. Locally, any trivialization of the torsor splits the exact sequence $0 \rightarrow \mathfrak{l} \otimes \mathcal{O}_{X} \rightarrow \widetilde{\mathcal{T}}_{X} \rightarrow \mathcal{T}_{X} \rightarrow 0$ and gives $\widetilde{\mathcal{D}}_{X} \cong \mathcal{D}_{X} \otimes \mathrm{U}(\mathfrak{l})$. So the map $\mathrm{U}(\mathfrak{l}) \rightarrow \widetilde{\mathcal{D}}_{X}$ given by the $L$-action is an embedding, $\widetilde{\mathcal{D}}_{X}$ is a locally free $\mathrm{U}(\mathfrak{l})$-module, and the center $Z[\mathrm{U}(\mathfrak{l})]$ of $\mathrm{U}(\mathfrak{l})$ is central in $\widetilde{\mathcal{D}}_{X}$. Recall that $\mathcal{Z}(\mathrm{U}(\mathfrak{l}))=\mathfrak{l}^{*(1)} \times_{\mathfrak{h}^{*(1)} / W_{L}} \mathfrak{h}^{*} / W_{L}$ for the Cartan group $H$ and the Weyl group $W_{L}$ of $L$ (Theorem 1.1.5). Since $U(\mathfrak{l})$ is free over its Harish-Chandra center $\mathfrak{Z}_{\mathrm{HC}}(\mathfrak{l})=\mathrm{U}(\mathfrak{l})^{L} \stackrel{\cong}{\cong} \mathcal{O}\left(\mathfrak{h}^{*} / W_{L}\right)$, it follows that $\widetilde{\mathcal{D}}_{X}$ is locally free over $\mathfrak{Z}_{\mathrm{HC}}(\mathfrak{l})$. We consider the specializations $\mathcal{D}_{X}^{\lambda} \stackrel{\text { def }}{=} \widetilde{\mathcal{D}}_{X} \otimes_{\mathfrak{Z}_{\mathrm{HC}}} \mathbb{k}_{\lambda}$. Notice that the augmentation $\mathrm{U}(\mathfrak{l}) \rightarrow \mathbb{k}$ gives $\widetilde{\mathcal{D}}_{X} \otimes_{\mathrm{U}(\mathfrak{l})} \mathbb{k} \stackrel{\cong}{\longrightarrow} \mathcal{D}_{X}$.

The center $\mathcal{O}_{T^{*} \widetilde{X}^{(1)}}$ of $\mathcal{D}_{\widetilde{X}}$ gives another central subalgebra $\left(\pi_{*} \mathcal{O}_{T^{*} \widetilde{X}^{(1)}}\right)^{L}=\mathcal{O}_{\widetilde{T}^{*} X^{(1)}}$ of $\widetilde{\mathcal{D}}_{X}$. We combine two central subalgebras into a map from functions on $\widetilde{T}^{*} X^{(1)} \times_{\mathfrak{l}^{*}(1)} \mathcal{Z}(\mathrm{U}(\mathfrak{l}))=\widetilde{T}^{*} X^{(1)} \times_{\mathfrak{h}^{*(1)} / W_{L}} \mathfrak{h}^{*} / W_{L}$ to the center of $\widetilde{\mathcal{D}}_{X}$ (we use the exact sequence $0 \rightarrow \mathfrak{l} \otimes \mathcal{O}_{X} \rightarrow \widetilde{\mathcal{T}}_{X} \rightarrow$ $\left.\mathcal{T}_{X} \rightarrow 0\right)$. The above local trivializations now show that this is an isomorphism, i.e., $\mathcal{Z}\left(\widetilde{\mathcal{D}}_{X}\right)=\widetilde{T}^{*} X^{(1)} \times_{\mathfrak{h}^{*(1)} / W_{L}} \mathfrak{h}^{*} / W_{L}$. Moreover we also see that the Azumaya locus of $\widetilde{\mathcal{D}}_{X}$ in $\mathcal{Z}\left(\widetilde{\mathcal{D}}_{X}\right)$ is the fibered product over $\mathfrak{l}^{*(1)}$ of $\widetilde{T}^{*} X^{(1)}$ and the Azumaya locus of $\mathrm{U}(\mathfrak{l})$. This implies that the Azumaya locus in $\mathcal{Z}\left(\widetilde{\mathcal{D}}_{X}\right)$ is precisely the smooth part of $\mathcal{Z}\left(\widetilde{\mathcal{D}}_{X}\right)$ (use 1.1.7). For any $\lambda \in \mathfrak{h}^{*}$ the center of the specialization $\mathcal{D}_{X}^{\lambda}$ contains the algebra of functions on $\mathcal{Z}\left(\widetilde{\mathcal{D}}_{X}\right) \times_{\mathfrak{h}^{*} / W_{L}} \lambda=\widetilde{T}^{*} X^{(1)} \times_{\mathfrak{h}^{*(1)} / W_{L}} \operatorname{AS}(\lambda)$. This is a torsor for $T^{*} X^{(1)}$ over the quotient $X^{(1)} \times\left(\mathfrak{l}^{*(1)} \times_{\mathfrak{l}^{*}(1)} / W_{L} \mathrm{AS}(\lambda)\right)$. In particular, by using 1.1 .7 for the group $L$ we see that for any $L$-unramified weight $\lambda \in \mathfrak{h}_{\mathrm{unr}}^{*}(L)$, the sheaf $\mathcal{D}_{X}^{\lambda}$ is an Azumaya algebra on $\widetilde{\mathcal{Z}} \times_{\mathfrak{h}^{*} / W_{L}} \lambda$. 
For instance if $\lambda=d(\phi)$ is the differential of a character $\phi$ of $L$ then $\operatorname{AS}(\lambda)=0$ and $\mathcal{D}_{X}^{\lambda}$ is identified with the sheaf $\mathcal{O}_{\phi} \mathcal{D}_{X} \stackrel{\text { def }}{=} \mathcal{O}_{\phi} \otimes \mathcal{D}_{X} \otimes \mathcal{O}_{\phi}{ }^{-1}$ of differential operators on sections of the line bundle $\mathcal{O}_{\phi}$ on $X$, associated to the $L$-torsor $\widetilde{X}$ and a character $\phi$ of $L$. The following fact (which will not be used in the sequel) is proved in the same way as the version in [BMR, Lemma 2.3.1].

Lemma. Let $\nu \in \mathfrak{I}^{*}$ be the differential of some character of L. Define a morphism $\tau_{\nu}$ from $\widetilde{T}^{*} X^{(1)} \times_{\mathfrak{t}^{*}(1)} \mathfrak{t}^{*}$ to itself by $\tau_{\nu}(x, \lambda)=(x, \lambda+\nu)$. The Azumaya loci of algebras $\widetilde{\mathcal{D}}_{X}$ and $\tau_{\nu}^{*}\left(\widetilde{\mathcal{D}}_{X}\right)$ are the same, and the corresponding restrictions are equivalent Azumaya algebras. More precisely, the choice of $\eta$ with $\nu=d \eta$ determines an equivalence between the two Azumaya algebras. The corresponding equivalence of the categories of modules is given by $\mathcal{M} \mapsto \mathcal{O}_{\mathcal{P}, \eta} \otimes_{\mathcal{O}_{\mathcal{P}}} \mathcal{M}$.

Remark. The equivalence of Azumaya algebras can also be deduced from Lemma 1.3.3 below.

\subsubsection{Algebras $\widetilde{\mathcal{D}}_{\mathcal{P}}$}

Let $\mathcal{P}$ be a partial flag variety and $\mathcal{B} \stackrel{a}{\rightarrow} \mathcal{P} \stackrel{b}{\rightarrow}$ pt. Here $P$ will always denote a parabolic subgroup $P \in \mathcal{P}$. Denote by $J$ its unipotent radical and let $\bar{P} \stackrel{\text { def }}{=} P / J$ be the Levi quotient of $P$. Its Lie algebra $\overline{\mathfrak{p}}$ has Cartan algebra $\mathfrak{h}$ and the Weyl group $W_{\mathcal{P}} \subseteq W$.

Over $\mathcal{P}=G / P$ there is a $\bar{P}$-torsor $\pi=\pi_{\mathcal{P}}: \widetilde{\mathcal{P}}=G / J \rightarrow \mathcal{P}$. As in 1.2.1 it yields an algebra $\widetilde{\mathcal{D}}_{\mathcal{P}} \stackrel{\text { def }}{=}\left(\pi_{*} \mathcal{D}_{\widetilde{\mathcal{P}}}\right)^{\bar{P}}$ and its specializations $\mathcal{D}^{\lambda} \mathcal{P} \stackrel{\text { def }}{=}$ $\widetilde{\mathcal{D}}_{\mathcal{P}} \otimes_{S(\mathfrak{h})^{W_{\mathcal{P}}}} \mathbb{k}_{\lambda}$ for $\lambda \in \mathfrak{h}^{*}$. The action of $G \times \bar{P}$ on $\widetilde{\mathcal{P}}=G / J$ by $(g, p) \cdot s J \stackrel{\text { def }}{=}$ $g s p^{-1} J$, differentiates to a map $\mathfrak{g} \oplus \overline{\mathfrak{p}} \rightarrow \widetilde{\mathcal{T}}_{\mathcal{P}}$ which extends to $\mathrm{U}(\mathfrak{g}) \otimes \mathrm{U}(\overline{\mathfrak{p}}) \rightarrow$ $\widetilde{\mathcal{D}}_{\mathcal{P}}$. When $\mathcal{P}$ is the full flag variety $\mathcal{B}$, then $\widetilde{\mathcal{D}}_{\mathcal{B}}$ is defined by the $H$-torsor $\widetilde{\mathcal{B}} \stackrel{\text { def }}{=} G / N \stackrel{\pi}{\rightarrow} \mathcal{B}$ and it is a deformation over $\mathfrak{h}^{*}$ of the ring of differential operators $\mathcal{D}_{\mathcal{B}}=\mathcal{D}_{\mathcal{B}}^{0}{ }^{(9)}$. When $P=G$ then $\mathcal{P}=$ pt and $\widetilde{\mathcal{D}}_{\mathcal{P}}=\mathrm{U}$.

The sheaf of algebras $\widetilde{\mathcal{D}}_{\mathcal{P}}$ carries a filtration induced by the canonical filtration on crystalline differential operators, cf. [BMR, 1.2]. The associated graded is canonically identified with the sheaf of regular functions on the

\footnotetext{
${ }^{9}$ We can use $\widetilde{\mathcal{D}}_{\mathcal{B}}$ to describe the Harish-Chandra map (1.1.5). The $H$-action on $\widetilde{\mathcal{B}}$ gives an isomorphism $\mathrm{U}(\mathfrak{h}) \stackrel{\cong}{\rightrightarrows} \Gamma\left(\mathcal{B}, \widetilde{\mathcal{D}}_{\mathcal{B}}\right)^{G}$, and then the $G$-action gives the map $\mathrm{U}^{G} \rightarrow$ $\Gamma\left(\mathcal{B}, \widetilde{\mathcal{D}}_{\mathcal{B}}\right)^{G} \cong S(\mathfrak{h})$ which gives an isomorphism $\mathrm{U}^{G} \stackrel{i_{\mathrm{HC}}}{\longrightarrow} S(\mathfrak{h})^{(W, \bullet)}$.
} 
$T^{*} \mathcal{P}$-torsor over $G \times_{P} \overline{\mathfrak{p}}^{*}$, which is defined by: $\widetilde{\mathfrak{g}}_{\mathcal{P}}^{*} \stackrel{\text { def }}{=} \widetilde{T}^{*} \mathcal{P}=\{(\mathfrak{p}, \chi) \in \mathcal{P} \times$ $\left.\mathfrak{g}^{*},\left.\chi\right|_{\text {nil }(\mathfrak{p})}=0\right\}$. For $\mathcal{P}=\mathcal{B}$ we use a simplified notation $\widetilde{\mathfrak{g}}^{*}=\widetilde{\mathfrak{g}}_{\mathcal{B}}^{*}$. We have projections $\operatorname{pr}_{\mathfrak{g}}: \widetilde{\mathfrak{g}}_{\mathcal{P}}^{*} \rightarrow \mathfrak{g}^{*}, \operatorname{pr}_{\mathfrak{g}}(\mathfrak{p}, \chi)=\chi$ and $\operatorname{pr}_{\overline{\mathfrak{p}}}: \widetilde{\mathfrak{g}}_{\mathcal{P}}^{*} \rightarrow \overline{\mathfrak{p}}^{*} / \bar{P}=\mathfrak{h}^{*} / W_{\mathcal{P}}$ which sends $(\mathfrak{p}, \chi)$ to the coadjoint image of $\left.\chi\right|_{\mathfrak{p}} \in(\mathfrak{p} / \operatorname{nil}(\mathfrak{p}))^{*}=\overline{\mathfrak{p}}^{*}$; they yield a map pr $=\operatorname{pr}_{\mathfrak{g}} \times \operatorname{pr}_{\overline{\mathfrak{p}}}: \widetilde{\mathfrak{g}}_{\mathcal{P}}^{*} \rightarrow \mathfrak{g}^{*} \times \mathfrak{h}^{*} / W \mathfrak{h}^{*} / W_{\mathcal{P}}$ (we use the canonical isomorphism $\left.\mathfrak{g}^{*} / G \rightarrow \mathfrak{h}^{*} / W\right)$.

1.2.3. Cohomology of $\widetilde{\mathcal{D}}_{\mathcal{P}}$ and Azumaya property

Define the open subset $\mathfrak{h}_{\mathcal{P} \text {-unr }} \subseteq \mathfrak{h}^{*}$ of $\mathcal{P}$-unramified weights as $\mathfrak{h}_{\mathcal{P} \text {-unr }}^{*} \stackrel{\text { def }}{=} \mathfrak{h}_{\text {unr }}^{*}(\bar{P})$, i.e., weights that are unramified for the Levi group $\bar{P}$ of $P$. By definition in 1.1.6, this means that the map $\mathfrak{h}^{*} / W_{\mathcal{P}} \rightarrow \mathfrak{h}^{*} / W$ is unramified at $W_{\mathcal{P}} \bullet \lambda$. The condition is $\left(W_{\mathcal{P}}\right)_{\lambda}=\left(W_{\mathcal{P}}\right)_{\mathrm{AS}(\lambda)}$, or equivalently $\langle\check{\alpha}, \lambda+\rho\rangle \notin \mathbb{F}_{p}^{*}$ for roots $\alpha$ in $\Delta_{\mathcal{P}}$. It is clear that $\mathfrak{h}_{\mathcal{P}-\text { unr }}^{*}$ contains the set of weights with singularity of type $\mathcal{P}$, i.e., with the stabilizer equal to $W_{\mathcal{P}}$. Let us also define the unramified part of the central variety of $\widetilde{\mathcal{D}}_{\mathcal{P}}$ as the open subvariety

$$
\mathcal{Z}\left(\widetilde{\mathcal{D}}_{\mathcal{P}}\right)_{\text {unr }} \stackrel{\text { def }}{=} \mathcal{Z}\left(\widetilde{\mathcal{D}}_{\mathcal{P}}\right) \times_{\mathfrak{h}^{*} / W_{\mathcal{P}}}\left(\mathfrak{h}_{\mathcal{P}-\text { unr }}^{*} / W_{\mathcal{P}}\right) \subseteq \mathcal{Z}\left(\widetilde{\mathcal{D}}_{\mathcal{P}}\right)
$$

In particular, $\mathcal{Z}(\mathrm{U})$ unr $\stackrel{\text { def }}{=} \mathfrak{g}^{*(1)} \times_{\mathfrak{h}^{*(1)} / W} \mathfrak{h}_{\mathrm{unr}}^{*} \subseteq \mathcal{Z}(\mathrm{U})$.

Proposition. (a) $\mathrm{R} a_{*} \widetilde{\mathcal{D}}_{\mathcal{B}} \cong \widetilde{\mathcal{D}}_{\mathcal{P}} \otimes_{S(\mathfrak{h})^{W_{\mathcal{P}}}} S(\mathfrak{h})$ canonically, hence $\widetilde{\mathcal{D}}_{\mathcal{P}} \cong \widetilde{\mathcal{D}}_{\mathcal{B}}^{W_{\mathcal{P}}}$. For each $\lambda \in \mathfrak{h}^{*}, \operatorname{R} a_{*} \mathcal{D}_{\mathcal{B}}^{\lambda} \cong \mathcal{D}_{\mathcal{P}}^{\lambda}$.

(b) The map $\mathrm{U}(\mathfrak{g}) \otimes S(\mathfrak{h})^{W_{\mathcal{P}}} \rightarrow \Gamma\left(\mathcal{P}, \widetilde{\mathcal{D}}_{\mathcal{P}}\right)$ factors through $\widetilde{\mathrm{U}}^{W_{\mathcal{P}}}=$ $\mathrm{U} \otimes_{S(\mathfrak{h})^{W}} S(\mathfrak{h})^{W_{\mathcal{P}}}$ and yields an isomorphism $\widetilde{\mathrm{U}}^{W_{\mathcal{P}}} \stackrel{\cong}{\mathrm{R}} \Gamma\left(\widetilde{\mathcal{D}}_{\mathcal{P}}\right)$. Also $\mathrm{U}^{\lambda} \stackrel{\cong}{\cong} \Gamma\left(\mathcal{D}_{\mathcal{P}}^{\lambda}\right)$ for $\lambda \in \mathfrak{h}^{*}$.

(c) The center of $\widetilde{\mathcal{D}}_{\mathcal{P}}$ is the sheaf of functions on $\mathcal{Z}\left(\widetilde{\mathcal{D}}_{\mathcal{P}}\right)=$ $\tilde{\mathfrak{g}}_{\mathcal{P}}^{*}{ }^{(1)} \times_{\mathfrak{h}^{*(1)} / W_{\mathcal{P}}} \mathfrak{h}^{*} / W_{\mathcal{P}}$. The algebra $\widetilde{\mathcal{D}}_{\mathcal{P}}$ is Azumaya over the open subvariety $\mathcal{Z}\left(\widetilde{\mathcal{D}}_{\mathcal{P}}\right)_{\text {unr }} \subseteq \mathcal{Z}\left(\widetilde{\mathcal{D}}_{\mathcal{P}}\right)$.

(d) Consider the canonical map $\mathcal{P}=G / P \rightarrow \mathcal{Q}=G / Q, P \subseteq Q$ between two partial flag varieties. It induces a map of central varieties $\varpi_{\mathcal{Q}}^{\mathcal{P}}: \mathcal{Z}\left(\widetilde{\mathcal{D}}_{\mathcal{P}}\right) \rightarrow \mathcal{Z}\left(\widetilde{\mathcal{D}}_{\mathcal{Q}}\right)$, i.e., $\varpi_{\mathcal{Q}}^{\mathcal{P}}: \widetilde{\mathfrak{g}}_{\mathcal{P}}^{*}{ }^{(1)} \times_{\mathfrak{h}^{*(1)} / W_{\mathcal{P}}} \mathfrak{h}^{*} / W_{\mathcal{P}} \rightarrow \widetilde{\mathfrak{g}}_{\mathcal{Q}}^{*}{ }^{(1)} \times_{\mathfrak{h}^{*(1)} / W_{\mathcal{Q}}}$ $\mathfrak{h}^{*} / W_{\mathcal{Q}} ;$ in particular, for $\mathcal{Q}=$ pt we get

$$
\begin{array}{rl}
\varpi^{\mathcal{P}} \stackrel{\text { def }}{=} \varpi_{\mathrm{pt}}^{\mathcal{P}}: \mathcal{Z}\left(\widetilde{\mathcal{D}}_{\mathcal{P}}\right)=\widetilde{\mathfrak{g}}_{\mathcal{P}}^{*}(1) \times_{\mathfrak{h}^{*(1)} / W_{\mathcal{P}}} \mathfrak{h}^{*} / W_{\mathcal{P}} & \mathcal{Z}(\mathrm{U}) \\
& =\mathfrak{g}^{*(1)} \times_{\mathfrak{h}^{*(1)} / W} \mathfrak{h}^{*} / W .
\end{array}
$$


Then the preimage of the Azumaya locus is contained in the Azumaya locus, i.e.,

$$
\left(\varpi_{\mathcal{Q}}^{\mathcal{P}}\right)^{-1}\left(\mathcal{Z}\left(\widetilde{\mathcal{D}}_{\mathcal{Q}}\right)_{\mathrm{AZ}}\right) \subset \mathcal{Z}\left(\widetilde{\mathcal{D}}_{\mathcal{P}}\right)_{\mathrm{AZ}}
$$

and moreover the two Azumaya algebras are canonically related via the pullback:

$$
\left.\widetilde{\mathcal{D}}_{\mathcal{P}}\right|_{\left(\varpi_{\mathcal{Q}}^{\mathcal{P}}\right)^{-1}\left(\mathcal{Z}\left(\widetilde{\mathcal{D}}_{\mathcal{Q}}\right)_{\mathrm{AZ}}\right)} \cong\left(\varpi_{\mathcal{Q}}^{\mathcal{P}}\right)^{*}\left(\widetilde{\mathcal{D}}_{\mathcal{Q}}\right)
$$

(e) The complement to $\left(\varpi^{\mathcal{P}}\right)^{-1}\left(\mathcal{Z}(\mathrm{U})_{\mathrm{AZ}}\right)$ in $\mathcal{Z}\left(\widetilde{\mathcal{D}}_{\mathcal{P}}\right)$ has codimension at least two for every $\mathcal{P}$.

Proof. The first claim in (a) implies the rest of (a), because the derived global sections functor commutes with the derived tensor product over a ring of global endomorphisms, while the $\operatorname{ring} S(\mathfrak{h})$ acts locally freely both on $\widetilde{\mathcal{D}}_{\mathcal{B}}$ and on $\widetilde{\mathcal{D}}_{\mathcal{P}} \otimes_{S(\mathfrak{h}){ }^{W_{\mathcal{P}}}} S(\mathfrak{h})$ by 1.2 .1 .

For a semisimple group $G$ and $\mathcal{P}=$ pt the first claim in (a) has been established in [BMR, Proposition 3.4.1]. A very similar argument shows that the first claim holds for $\mathcal{P}=$ pt and a reductive group $G$. We proceed to deduce the general case. For a fixed $P \in \mathcal{P}$ choose a Levi factor $L$ of $P$ and let $P_{-}=L J_{-}$be the corresponding opposite parabolic. The maps $\widetilde{\mathcal{B}} \rightarrow \mathcal{B} \stackrel{a}{\rightarrow} \mathcal{P} \leftarrow \widetilde{\mathcal{P}}$, restricted to the neighborhood $\mathcal{U}=J_{-} \cdot \mathfrak{p}$ of $\mathfrak{p} \in \mathcal{P}$, are naturally identified with $\mathcal{U} \times \widetilde{\mathcal{B}}(\bar{P}) \rightarrow \mathcal{U} \times \mathcal{B}(\bar{P}) \rightarrow \mathcal{U} \times$ pt $\leftarrow \mathcal{U} \times \bar{P}$, where $\mathcal{B}(\bar{P})$ is flag variety of $\bar{P}$.

Therefore $\mathcal{D}_{\widetilde{\mathcal{P}}} \mid \varpi^{\mathcal{P}-1} \mathcal{U} \cong \mathcal{D}_{\mathcal{U}} \otimes \mathcal{D}_{\bar{P}}$ and $\widetilde{\mathcal{D}}_{\mathcal{P}} \mid \mathcal{U} \cong \mathcal{D}_{\mathcal{U}} \otimes \mathcal{D}(\bar{P})^{\bar{P} \times 1}=$ $\mathcal{D}_{\mathcal{U}} \otimes \mathrm{U}(\overline{\mathfrak{p}})$. Now, equality $\mathcal{D}_{\widetilde{\mathcal{B}}} \mid a^{-1} \mathcal{U} \cong \mathcal{D}_{\mathcal{U}} \otimes \widetilde{\mathcal{D}}_{\mathcal{B}(\bar{P})}$ shows that on $\mathcal{U}$ :

$$
\begin{aligned}
\mathrm{R} a_{*} \widetilde{\mathcal{D}}_{\mathcal{B}} & \cong \mathcal{D}_{\mathcal{U}} \otimes_{\mathbb{k}} \mathrm{R} \Gamma\left[\mathcal{B}(\bar{P}), \widetilde{\mathcal{D}}_{\mathcal{B}(\bar{P})}\right]=\mathcal{D}_{\mathcal{U}} \otimes_{\mathbb{k}} \widetilde{\mathrm{U}}(\overline{\mathfrak{p}}) \\
& =\left[\mathcal{D}_{\mathcal{U}} \otimes_{\mathbb{k}} \mathrm{U}(\overline{\mathfrak{p}})\right] \otimes_{S(\mathfrak{h})^{W_{\mathcal{P}}}} S(\mathfrak{h}) \cong \widetilde{\mathcal{D}}_{\mathcal{P}} \otimes_{S(\mathfrak{h})^{W_{\mathcal{P}}}} S(\mathfrak{h})
\end{aligned}
$$

Similarly, in (b) the second sentence follows from the first one. The case $\mathcal{P}=\mathcal{B}$ follows from [BMR, Proposition 3.4.1], where it is stated for semisimple $G$. Thus we have: $\operatorname{R} \Gamma\left(\mathcal{P} ; \operatorname{R} a_{*} \widetilde{\mathcal{D}}_{\mathcal{B}}\right)=\mathrm{R}(b a)_{*} \widetilde{\mathcal{D}}_{\mathcal{B}} \cong \mathrm{U} \otimes_{S(\mathfrak{h})^{W}} S(\mathfrak{h})$, where $b$ denotes the projection $\mathcal{P} \rightarrow$ pt. Substituting the description of $\mathrm{R}(b a)_{*} \widetilde{\mathcal{D}}_{\mathcal{B}}$ from (a) we see that the morphism $\mathrm{U} \otimes_{S(\mathfrak{h}){ }^{W}} S(\mathfrak{h})^{W_{\mathcal{P}}} \rightarrow \mathrm{R} b_{*} \widetilde{\mathcal{D}}_{\mathcal{P}}$ becomes an isomorphism after tensoring with $S(\mathfrak{h})$ over $S(\mathfrak{h})^{W_{\mathcal{P}}}$. Since $S(\mathfrak{h})$ is free over $S(\mathfrak{h})^{W_{\mathcal{P}}}$ by [De], this implies the first sentence in (b).

(c) has already been observed in 1.2.1. 
Claim (d) in the particular case $\mathcal{P}=\mathcal{B}, \mathcal{Q}=$ pt and $G$ semisimple is in [BMR, Proposition 5.2.1]. The general case reduces to this one as in the proof of (b).

(e) follows from the fact that $(\chi, \lambda) \in \mathcal{Z}(\mathrm{U})$ lies in $\mathcal{Z}(\mathrm{U})_{\mathrm{AZ}}$ provided that $\chi$ is regular (not necessarily semisimple) [BG, Theorems 2.5, 2.6, Corollary 3.4], and that the preimage of regular elements in $\widetilde{\mathfrak{g}}^{*}$ has complement of codimension two. The latter claim is equivalent to non-regular elements forming a subset of codimension two in a fixed Borel subalgebra $\mathfrak{b} \subset \mathfrak{g}$. Writing $\mathfrak{b}=\mathfrak{h} \oplus \mathfrak{n}$ we see that the set of non-regular elements is contained in the union of $\mathfrak{h}_{\alpha} \times \mathfrak{n}$, where $\mathfrak{h}_{\alpha}$ runs over the set of root hyperplanes. Moreover, $\mathfrak{h}_{\alpha} \times \mathfrak{n}$ is not contained in the set of non-regular elements for any root hyperplane $\mathfrak{h}_{\alpha}$, which implies the bound on the codimension.

Remarks 1.2.4. (0) $\mathcal{Z}\left(\widetilde{\mathcal{D}}_{\mathcal{P}}\right)$ consists of all $\left(\mathfrak{p}^{(1)}, \chi^{(1)}, W_{\mathcal{P}} \bullet \lambda\right) \in \mathcal{P}^{(1)} \times$ $\mathfrak{g}^{*(1)} \times \mathfrak{h}^{*} / W_{\mathcal{P}}$, such that (i) $\chi \perp \mathfrak{u}$ (so that the restriction $\chi \mid \mathfrak{p}$ factors to $\chi_{\mathfrak{p}} \in \overline{\mathfrak{p}}^{*}$ ), and (ii) the image of $\chi_{\mathfrak{p}}{ }^{(1)}$ in $\left(\overline{\mathfrak{p}}^{*} / \bar{P}\right)^{(1)} \cong \mathfrak{h}^{*(1)} / W_{\mathcal{P}}$ is the orbit $W_{\mathcal{P}} \nu$ for $\nu=\operatorname{AS}(\lambda)$. Here, (i) means that $\mathfrak{p}$ lies in the (generalized) Springer fiber $\mathcal{P}_{\chi} \stackrel{\text { def }}{=} \widetilde{\mathfrak{g}}_{\mathcal{P}}^{*} \times \mathfrak{g}^{*} \chi$.

(1) The fibers of $\widetilde{\mathcal{D}}_{\mathcal{P}}$ and $\mathcal{D}_{\mathcal{P}}^{\lambda}$ at $P \in \mathcal{P}$ are the induced g-modules $\operatorname{Ind}_{U(\mathfrak{p})}^{U(\mathfrak{g})} \widetilde{U}(\overline{\mathfrak{p}})$ and $\operatorname{Ind}_{U^{\lambda}(\mathfrak{p})}^{U^{\lambda}(\mathfrak{g})} U^{\lambda}(\overline{\mathfrak{p}})$ (see the proof of (a) above). The fiber of $\widetilde{\mathcal{D}}_{\mathcal{P}}$ at a point $\left(\mathfrak{p}^{(1)}, \chi^{(1)}, W_{\mathcal{P}} \bullet \lambda\right)$ of $\mathcal{Z}\left(\widetilde{\mathcal{D}}_{\mathcal{P}}\right)$ is $\operatorname{Ind}_{u_{\chi}(\mathfrak{p})}^{u_{\chi}(\mathfrak{g})} \mathrm{U}_{\chi}^{\lambda}(\overline{\mathfrak{p}})$.

(2) $\mathcal{Z}\left(\widetilde{\mathcal{D}}_{\mathcal{P}}\right)$ is a torsor for the twisted cotangent bundle $T^{*} \mathcal{P}^{(1)}=\left(G \times{ }_{P}\right.$ $\left.\mathfrak{p}^{\perp}\right)^{(1)}$ over the quotient $\left(G \times{ }_{P} \overline{\mathfrak{p}}^{*}\right)^{(1)} \times_{\mathfrak{h}^{*(1)} / W_{\mathcal{P}}} \mathfrak{h}^{*} / W_{\mathcal{P}}=G \times_{P}\left[\overline{\mathfrak{p}}^{*(1)} \times_{\mathfrak{h}^{*(1)} / W_{\mathcal{P}}}\right.$ $\left.\mathfrak{h}^{*} / W_{\mathcal{P}}\right]=G \times_{P} \mathcal{Z}(\mathrm{U}(\overline{\mathfrak{p}}))$.

(3) The classical limit of the claims (a) and (b) above is the observation that the canonical maps $\widetilde{\mathfrak{g}}_{\mathcal{B}}^{*} \rightarrow \widetilde{\mathfrak{g}}_{\mathcal{P}}^{*} \times_{\mathfrak{h}^{*} / W_{\mathcal{P}}} \mathfrak{h}^{*}$ and $\widetilde{\mathfrak{g}}_{\mathcal{P}}^{*} \rightarrow \mathfrak{g}^{*} \times_{\mathfrak{h}^{*}} / W_{\mathcal{P}} \mathfrak{h}^{*} / W_{\mathcal{P}}$ are affinizations over $\mathcal{P}$ and over the point respectively. On the level of centers we get that $\mathcal{Z}\left(\widetilde{\mathcal{D}}_{\mathcal{P}}\right) \rightarrow \mathcal{Z}(\widetilde{\mathrm{U}}) / W_{\mathcal{P}}=\mathfrak{g}^{*(1)} \times_{\mathfrak{h}^{*(1)} / W} \mathfrak{h}^{*} / W_{\mathcal{P}}$ is an affinization.

\subsection{Splitting on parabolic Springer fibers}

Here we generalize the observation from [BMR] that the equivalence class of the Azumaya algebra on the (twisted) cotangent bundle to $\mathcal{B}$ is the pull-back of a certain class under the Springer map. This implies, in particular, that when $\mathcal{D}_{\mathcal{P}}^{\lambda}$ is an Azumaya algebra, i.e., $\lambda \in \mathfrak{h}_{\mathcal{P} \text {-unr }}^{*}$, then this Azumaya algebra splits on formal neighborhoods of parabolic Springer fibers $\mathcal{P}_{\chi, \mathrm{AS}(\lambda)}{ }^{(1)}$ in $\mathcal{Z}\left(\widetilde{\mathcal{D}}_{\mathcal{P}}\right)$. 
1.3.1. An auxiliary etale covering of $\mathfrak{h}^{*}$

We need some notations. Consider the addition map $\mathfrak{h}_{\mathrm{unr}}^{*} \times \mathfrak{h}_{\mathbb{F}_{p}}^{*}=$ $\bigcup_{\lambda_{\mathbb{F}_{p}} \in \mathfrak{h}_{\mathbb{F}_{p}}^{*}} \mathfrak{h}_{\text {unr }}^{*} \rightarrow \mathfrak{h}^{*},\left(\lambda_{\text {unr }}, \lambda_{\mathbb{F}_{p}}\right) \mapsto \lambda_{\text {unr }}+\lambda_{\mathbb{F}_{p}}$ and the induced maps on the quotients: $a_{\mathcal{P}}:\left(\mathfrak{h}_{\mathrm{unr}}^{*} \times \mathfrak{h}_{\mathbb{F}_{p}}^{*}\right) / W_{\mathcal{P}} \rightarrow \mathfrak{h}^{*} / W_{\mathcal{P}}$. Notice that the action of $W$ on $\mathfrak{h}_{\mathrm{unr}}^{*}$ and $\mathfrak{h}^{*}$ is the dot-action but the action on $\mathfrak{h}_{\mathbb{F}_{p}}^{*}$ is the usual action. When $P=G$, i.e., $\mathcal{P}=$ pt then $W_{\mathrm{pt}}=W$ and $a \stackrel{\text { def }}{=} a_{\mathrm{pt}}:\left(\mathfrak{h}_{\mathrm{unr}}^{*} \times \mathfrak{h}_{\mathbb{F}_{p}}^{*}\right) / W \rightarrow \mathfrak{h}^{*} / W$.

Lemma. a) For every partial flag variety $\mathcal{P}$ the map $a_{\mathcal{P}}$ is an etale covering.

b) Consider the two maps $\mathrm{pr}_{1}, a_{\mathcal{P}}:\left(\mathfrak{h}_{\mathrm{unr}}^{*} \times \mathfrak{h}_{\mathbb{F}_{p}}^{*}\right) / W_{\mathcal{P}} \rightarrow \mathfrak{h}^{*} / W_{\mathcal{P}}$. Their compositions with the Artin-Schreier map $\mathrm{AS} / W_{\mathcal{P}}$ are equal.

Proof. a) To check that the map is etale it suffices to check the equality of stabilizers $\operatorname{Stab}_{W}\left(\lambda_{\text {unr }}, \lambda_{\mathbb{F}_{p}}\right)=\operatorname{Stab}_{W}\left(\lambda_{\text {unr }}+\lambda_{\mathbb{F}_{p}}\right)$ for every $\left(\lambda_{\text {unr }}, \lambda_{\mathbb{F}_{p}}\right) \in$ $\mathfrak{h}_{\text {unr }}^{*} \times \mathfrak{h}_{\mathbb{F}_{p}}^{*}$. In view of Lemma 1.1.3, the stabilizer of $\lambda \in \mathfrak{h}^{*}$ is generated by reflections $s_{\alpha}$ where $\alpha$ runs over coroots such that $\langle\alpha, \lambda+\rho\rangle=0$. By the definition of an unramified element of $\mathfrak{h}^{*}$ for every coroot $\alpha$ we either have $\langle\alpha, \lambda+\rho\rangle \notin \mathbb{F}_{p}$, in which case $\left\langle\alpha, \lambda_{\text {unr }}+\lambda_{\mathbb{F}_{p}}+\rho\right\rangle \neq 0$; or $\left\langle\alpha, \lambda_{\text {unr }}+\rho\right\rangle=$ 0 . Thus $\operatorname{Stab}_{W}\left(\lambda_{\text {unr }}\right) \supseteq \operatorname{Stab}_{W}\left(\lambda_{\text {unr }}+\lambda_{\mathbb{F}_{p}}\right)$, hence $\operatorname{Stab}_{W}\left(\lambda_{\text {unr }}, \lambda_{\mathbb{F}_{p}}\right)=$ $\operatorname{Stab}_{W}\left(\lambda_{\text {unr }}+\lambda_{\mathbb{F}_{p}}\right)$.

It remains to show that $a_{\mathcal{P}}$ is a covering, thus we need to check that $\mathfrak{h}^{*}=\mathfrak{h}_{\mathrm{unr}}^{*}+\mathfrak{h}_{\mathbb{F}_{p}}^{*}$. Pick $\lambda \in \mathfrak{h}^{*}$. We claim that there exists $\lambda_{\mathbb{F}_{p}} \in \mathfrak{h}_{\mathbb{F}_{p}}^{*}$ such that $\langle\alpha, \lambda+\rho\rangle=\left\langle\alpha, \lambda_{\mathbb{F}_{p}}\right\rangle$ for every coroot $\alpha$ such that $\langle\alpha, \lambda\rangle \in \mathbb{F}_{p}$; then $\lambda_{\text {unr }} \stackrel{\text { def }}{=} \lambda-\lambda_{\mathbb{F}_{p}}$ is unramified. To check the existence of $\lambda_{\mathbb{F}_{p}}$ notice that the conditions on $\lambda_{\mathbb{F}_{p}}$ constitute a finite collection of (non-homogeneous) linear equations on an element of $\mathfrak{h}_{\mathbb{F}_{p}}^{*}$; the equations are defined over $\mathbb{F}_{p}$ and have a solution $\lambda$ in $\mathfrak{h}^{*}$, i.e. they have a solution over a larger field of coefficients. By standard linear algebra they also have a solution over $\mathbb{F}_{p}$.

b) is clear, because $\operatorname{AS}\left(\lambda+\lambda_{\mathbb{F}_{p}}\right)=\operatorname{AS}(\lambda)$ for $\lambda_{\mathbb{F}_{p}} \in \mathfrak{h}_{\mathbb{F}_{p}}^{*}$.

1.3.2. Azumaya algebras on an etale covering of central varieties

We now use $a_{\mathcal{P}}$ to get coverings of the central varieties described in Proposition 1.2.3.c and Theorem 1.1.5. We set

$$
\begin{aligned}
\widetilde{\mathcal{Z}}\left(\widetilde{\mathcal{D}}_{\mathcal{P}}\right) & \stackrel{\text { def }}{=} \mathcal{Z}\left(\widetilde{\mathcal{D}}_{\mathcal{P}}\right) \times_{\mathfrak{h}^{*} / W_{\mathcal{P}}}\left(\mathfrak{h}_{\mathrm{unr}}^{*} \times \mathfrak{h}_{\mathbb{F}_{p}}^{*}\right) / W_{\mathcal{P}} \\
& =\widetilde{\mathfrak{g}}_{\mathcal{P}}^{*}(1) \times_{\mathfrak{h}^{*}(1) / W_{\mathcal{P}}}\left(\left(\mathfrak{h}_{\mathrm{unr}}^{*} \times \mathfrak{h}_{\mathbb{F}_{p}}^{*}\right) / W_{\mathcal{P}}\right) .
\end{aligned}
$$


We also let $\widetilde{\mathcal{Z}}\left(\widetilde{\mathcal{D}}_{\mathcal{P}}\right)_{\mathrm{AZ}}=\left(\mathrm{id} \times a_{\mathcal{P}}\right)^{-1}\left(\mathcal{Z}\left(\widetilde{\mathcal{D}}_{\mathcal{P}}\right)_{\mathrm{AZ}}\right)$ be the preimage of the Azumaya locus of $\widetilde{\mathcal{D}}_{\mathcal{P}}$.

Recall that $\varpi^{\mathcal{P}} \stackrel{\text { def }}{=} \varpi_{\mathrm{pt}}^{\mathcal{P}}: \mathcal{Z}\left(\widetilde{\mathcal{D}}_{\mathcal{P}}\right) \rightarrow \mathcal{Z}(\mathrm{U})$ and consider

$$
\begin{aligned}
& \mathcal{Z}\left(\widetilde{\mathcal{D}}_{\mathcal{P}}\right) \supseteq \mathcal{Z}\left(\widetilde{\mathcal{D}}_{\mathcal{P}}\right)_{\mathrm{AZ}} \stackrel{\operatorname{id} \times a_{\mathcal{P}}}{\longleftarrow} \widetilde{\mathcal{Z}}\left(\widetilde{\mathcal{D}}_{\mathcal{P}}\right)_{\mathrm{AZ}} \stackrel{\varpi^{\mathcal{P}} \times \mathrm{pr}_{1}}{\longrightarrow} \mathcal{Z}(\mathrm{U}) \times_{\mathfrak{h}^{*} / W} \mathfrak{h}_{\mathrm{unr}}^{*} / W \\
& \stackrel{\text { def }}{=} \mathcal{Z}(\mathrm{U})_{\mathrm{unr}} \subseteq \mathcal{Z}(\mathrm{U}) \text {, }
\end{aligned}
$$

where $\operatorname{pr}_{1}:\left(\mathfrak{h}_{\mathrm{unr}}^{*} \times \mathfrak{h}_{\mathbb{F}_{p}}^{*}\right) / W_{\mathcal{P}} \rightarrow \mathfrak{h}_{\mathrm{unr}}^{*} / W$ is the projection.

LEMma. We have a Morita equivalence of Azumaya algebras on $\widetilde{\mathcal{Z}}\left(\widetilde{\mathcal{D}}_{\mathcal{P}}\right)_{\mathrm{AZ}}\left(\right.$ in particular, on $\left.\widetilde{\mathcal{Z}}(\mathrm{U})_{\mathrm{AZ}}\right)$ :

$$
\left(\mathrm{id} \times a_{\mathcal{P}}\right)^{*}\left(\widetilde{\mathcal{D}}_{\mathcal{P}}\right) \sim\left(\varpi^{\mathcal{P}} \times \operatorname{pr}_{1}\right)^{*}(\mathrm{U})
$$

Before proving the lemma we provide a slightly more precise statement. Notice that the variety $\left(\mathfrak{h}^{*} \times \mathfrak{h}_{\mathbb{F}_{p}}^{*}\right) / W_{\mathcal{P}}$ is a union of connected components

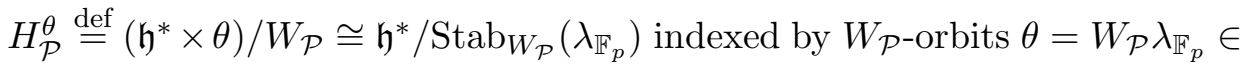
$\mathfrak{h}_{\mathbb{F}_{p}}^{*} / W_{\mathcal{P}}$ in $\mathfrak{h}_{\mathbb{F}_{p}}^{*}$.

We also get the corresponding components in the coverings of the central varieties: $\widetilde{\mathcal{Z}}\left(\widetilde{\mathcal{D}}_{\mathcal{P}}\right)^{\theta}=\mathcal{Z}\left(\widetilde{\mathcal{D}}_{\mathcal{P}}\right) \times_{\mathfrak{h}^{*} / W_{\mathcal{P}}} H_{\mathcal{P}}^{\theta}, \theta \in \mathfrak{h}_{\mathbb{F}_{p}}^{*} / W_{\mathcal{P}}$.

Lemma 1.3.3. For every $\theta \in \mathfrak{h}_{\mathbb{F}_{p}}^{*} / W_{\mathcal{P}}$ the choice of $\widetilde{\lambda} \in \Lambda$ such that $W_{\text {aff }}^{\prime} \bullet \widetilde{\lambda}$ equals $W \bullet \theta$ in $\Lambda / W_{\text {aff }}^{\prime}=\mathfrak{h}_{\mathbb{F}_{p}}^{*} / W$, defines an equivalence of Azumaya algebras on $\widetilde{\mathcal{Z}}\left(\widetilde{\mathcal{D}}_{\mathcal{P}}\right)^{\theta} \mathrm{AZ} \stackrel{\text { def }}{=} \widetilde{\mathcal{Z}}\left(\widetilde{\mathcal{D}}_{\mathcal{P}}\right)^{\theta} \cap \widetilde{\mathcal{Z}}\left(\widetilde{\mathcal{D}}_{\mathcal{P}}\right)_{\mathrm{AZ}}$,

$$
\left(\mathrm{id} \times a_{\mathcal{P}}\right)^{*}\left(\widetilde{\mathcal{D}}_{\mathcal{P}}\right) \sim\left(\varpi^{\mathcal{P}} \times \operatorname{pr}_{1}\right)^{*}(\mathrm{U})
$$

which satisfies the following properties:

(i) For any character $\mu$ of the Levi group $L$ the equivalence corresponding to $\widetilde{\lambda}^{\prime}=\widetilde{\lambda}+p \mu$ is the composition of the equivalence corresponding to $\widetilde{\lambda}$ and the twist by $\mathcal{O}_{\mathcal{P}^{(1)}, \mu}$.

(ii) Given a pair of partial flag varieties $\mathcal{P}=G / P \rightarrow \mathcal{Q}=G / Q$ for parabolics $P \subseteq Q$, the equivalence (2) is compatible with the isomorphism (1) from Proposition 1.2.3.d. 
Proof. To establish the equivalence of Azumaya algebras recall a well known fact (cf., e.g., [Mln, IV.2, Corollary 2.6]) that given two Azumaya algebras on a smooth variety over a field, every equivalence between their restrictions to a dense open subvariety extends to the whole variety; moreover, such an extension is unique provided that the open subvariety has complement of codimension at least two (on every component).

Thus it suffices to construct an equivalence between the two Azumaya algebras on such an open subset.

By Proposition 1.2.3.e the complement to the preimage of $\mathcal{Z}(\mathrm{U})_{\mathrm{AZ}}$ under the projection $\mathcal{Z}\left(\widetilde{\mathcal{D}}_{\mathcal{P}}\right) \rightarrow \mathcal{Z}(\mathrm{U})$ has codimension at least two. Thus it suffices to define $(2)$ on $\left(\mathrm{id} \times a_{\mathcal{P}}\right)^{-1}\left(\left(\varpi_{\mathrm{pt}}^{\mathcal{P}}\right)^{-1}\left(\mathcal{Z}(\mathrm{U})_{\mathrm{AZ}}\right)\right)$. In view of Proposition 1.2.3.d, the construction of the equivalence reduces to the case $\mathcal{P}=$ pt. Here

$$
\begin{aligned}
\mathcal{Z}(\mathrm{U}) \supseteq \mathcal{Z}(\mathrm{U})_{\mathrm{AZ}} \stackrel{\mathrm{id} \times a}{\longleftarrow} \widetilde{\mathcal{Z}}(\mathrm{U})_{\mathrm{AZ}} \stackrel{\varpi \times \mathrm{pr}_{1}}{\longrightarrow} \mathcal{Z}(\mathrm{U}) \times \mathfrak{h}^{*} / W \mathfrak{h}_{\mathrm{unr}}^{*} / W \\
\stackrel{\text { def }}{=} \mathcal{Z}(\mathrm{U})_{\mathrm{unr}} \subseteq \mathcal{Z}(\mathrm{U}),
\end{aligned}
$$

for the projection $\operatorname{pr}_{1}:\left(\mathfrak{h}_{\mathrm{unr}}^{*} \times \mathfrak{h}_{\mathbb{F}_{p}}^{*}\right) / W \rightarrow \mathfrak{h}_{\mathrm{unr}}^{*} / W$ and

$$
\widetilde{\mathcal{Z}}(\mathrm{U})=\mathcal{Z}(\mathrm{U}) \times \mathcal{Z}_{\mathrm{HC}}\left(\mathfrak{h}_{\mathrm{unr}}^{*} \times \mathfrak{h}_{\mathbb{F}_{p}}^{*}\right) / W=\mathfrak{g}^{*(1)} \times_{\mathfrak{h}^{*(1)} / W}\left(\left(\mathfrak{h}_{\mathrm{unr}}^{*} \times \mathfrak{h}_{\mathbb{F}_{p}}^{*}\right) / W\right)
$$

To define the required equivalence in this case we need to define for every $\theta \in \mathfrak{h}_{\mathbb{F}_{p}}^{*} / W$ with a fixed representative $\widetilde{\lambda} \in \Lambda$ a bimodule $M=M(\widetilde{\lambda})$ over U such that:

i) As a $\mathfrak{Z}_{\mathrm{Fr}}$ bimodule $M$ is concentrated on the diagonal, and as a $\mathfrak{Z}_{\mathrm{HC}}$ bimodule it is concentrated on $\left\{(\mu, \mu+d \widetilde{\lambda}) \bmod W \mid \mu \in \mathfrak{h}^{*}\right\} \hookrightarrow\left(\mathfrak{h}^{*} / W\right)^{2}=$ $\operatorname{Spec}\left(\mathfrak{Z}_{\mathrm{HC}}\right)^{2}$.

ii) The restriction of $M$ as a left $\mathrm{U}$ module to $\mathcal{Z}(\mathrm{U})_{\mathrm{unr}}$ is free of rank $|\theta|$ (notice that the cardinality $|\theta|$ of the orbit equals the degree of the map $\left.H^{\theta} \rightarrow \mathfrak{h}^{*} / W\right)$.

To construct such a module we observe that $\mathcal{O}_{\tilde{\lambda}} \widetilde{\mathcal{D}} \cong \widetilde{\mathcal{D}}$ so that $\widetilde{\mathcal{D}} \otimes \mathcal{O}_{\mathcal{B}}$ $\mathcal{O}_{-\widetilde{\lambda}}$ is a $\widetilde{\mathcal{D}}$-bimodule. Then the U-bimodule $\widetilde{M}=\Gamma\left(\mathcal{B}, \widetilde{\mathcal{D}} \otimes \mathcal{O}_{-\widetilde{\lambda}}\right)$ can be viewed as sections of $\widetilde{\mathcal{D}} \otimes \mathcal{O}_{-\widetilde{\lambda}}$ on $\mathcal{Z}(\widetilde{\mathcal{D}})$, and also (by removing a codimension two subvariety), as sections on $\widetilde{\mathfrak{g}}_{\text {reg }}^{*} \times_{\mathfrak{h}^{*(1)} / W} \mathfrak{h}^{*} / W$, where $\widetilde{\mathfrak{g}}_{\text {reg }}^{*}$ is the preimage of the subset of regular elements $\mathfrak{g}_{\text {reg }}^{*} \subset \mathfrak{g}^{*}$.

The natural map $\widetilde{\mathfrak{g}}_{\mathrm{reg}}^{*} \rightarrow \mathfrak{g}_{\mathrm{reg}}^{*} \times_{\mathfrak{h}^{*} / W} \mathfrak{h}^{*}$ is an isomorphism, thus the Weyl group $W$ acts on $\widetilde{\mathfrak{g}}^{*}$ reg. It is not hard to see that this action is 
compatible with the natural action on $\Lambda=\operatorname{Pic}(\mathcal{B}) \stackrel{\cong}{\longrightarrow} \operatorname{Pic}\left(\widetilde{\mathfrak{g}}_{\text {reg }}^{*}\right)$. Moreover, a line bundle $\mathcal{O}_{\widetilde{\mathfrak{g}}_{\mathrm{reg}}^{*}(1), \widetilde{\lambda}}$ carries an equivariant structure with respect to the subgroup $\operatorname{Stab}_{W}(\tilde{\lambda}) \subseteq W$. Here, $\operatorname{Stab}_{W}(\widetilde{\lambda}) \cong \operatorname{Stab}_{W}\left(\lambda_{\mathbb{F}_{p}}\right)$ in $W$ for some

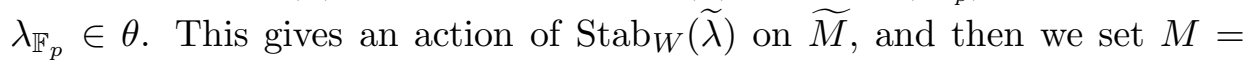
$\widetilde{M}^{\mathrm{Stab}_{W}(\widetilde{\lambda})}$. Properties (i) and (ii) are easy to check. This yields the required equivalence; the compatibilities then follow from the construction.

\subsubsection{Parabolic Springer fibers}

We proceed to describe the parabolic analogues of the Springer fibers and splitting of the Azumaya algebra on them.

Recall the maps $\mathfrak{g}^{*} \stackrel{\mathrm{pr}_{\mathfrak{g}}}{\longleftarrow} \widetilde{\mathfrak{g}}_{\mathcal{P}}^{*} \stackrel{\mathrm{pr}}{\longrightarrow} \mathfrak{g}^{*} \times \mathfrak{h}^{*} / W \mathfrak{h}^{*} / W_{\mathcal{P}}$ from 1.2.2 (here the $W$ action is not the dot action, but the standard one). For $(\chi, \nu) \in \mathfrak{g}^{*} \times_{\mathfrak{h}^{*} / W} \mathfrak{h}^{*}$ define parabolic Springer fibers $\mathcal{P}_{\chi}$ and $\mathcal{P}_{\chi, \nu}$ as subschemes $\operatorname{pr}_{\mathfrak{g}}{ }^{-1}(\chi)$ and $\operatorname{pr}^{-1}\left(\chi, W_{\mathcal{P}} \nu\right)$ of $\tilde{\mathfrak{g}}_{\mathcal{P}}^{*}$. Via the projection $\widetilde{\mathfrak{g}}_{\mathcal{P}}^{*} \stackrel{\tau}{\rightarrow} \mathcal{P}$ the parabolic Springer fiber $\mathcal{P}_{\chi, \nu}$ can be identified with a subscheme $\tau\left(\mathcal{P}_{\chi, \nu}\right)$ of $\mathcal{P}$, and $\mathcal{P}_{\chi, \nu}$ is a section of $\widetilde{\mathfrak{g}}_{\mathcal{P}}^{*}$ over $\tau\left(\mathcal{P}_{\chi, \nu}\right)$.

For $\lambda \in \mathfrak{h}^{*}$ denote $\nu \stackrel{\text { def }}{=} \operatorname{AS}(\lambda) \in \mathfrak{h}^{*(1)}$.

The fiber at $\left(\chi, W_{\mathcal{P}} \bullet \lambda\right)$ of the map of central varieties $\mathcal{Z}\left(\widetilde{\mathcal{D}}_{\mathcal{P}}\right) \rightarrow$ $\mathcal{Z}\left[\Gamma\left(\widetilde{\mathcal{D}}_{\mathcal{P}}\right)\right]$, i.e., of the map $\widetilde{\mathfrak{g}}_{\mathcal{P}}^{*(1)} \times_{\mathfrak{h}^{*(1)} / W_{\mathcal{P}}} \mathfrak{h}^{*} / W_{\mathcal{P}} \rightarrow \mathfrak{g}^{*(1)} \times_{\mathfrak{h}^{*(1)} / W} \mathfrak{h}^{*} / W_{\mathcal{P}}$, is the same as the parabolic Springer fiber $\mathcal{P}_{\chi, \nu}{ }^{(1)}$. Moreover, the formal neighborhoods of the fibers of the two maps are canonically identified. In other words, the formal neighborhood for $\mathcal{Z}\left(\widetilde{\mathcal{D}}_{\mathcal{P}}\right) \times_{\mathcal{Z}\left[\Gamma\left(\widetilde{\mathcal{D}}_{\mathcal{P}}\right)\right]}\left(\chi, W_{\mathcal{P}} \bullet \lambda\right) \subseteq$ $\mathcal{Z}\left(\widetilde{\mathcal{D}}_{\mathcal{P}}\right)$ can be identified with the formal neighborhood of $\widetilde{\mathfrak{g}}_{\mathcal{P}}^{*}{ }^{(1)} \times_{\mathfrak{g}^{*}(1)} \times \mathfrak{h}^{*} / W_{\mathcal{P}}$ $(\chi, \nu)=\mathcal{P}_{\chi, \nu}{ }^{(1)} \subseteq \widetilde{\mathfrak{g}}_{\mathcal{P}}^{*(1)}$.

COROllary. If $\lambda \in \mathfrak{h}_{\mathcal{P} \text {-unr }}^{*}$ then the Azumaya algebra obtained by restricting $\widetilde{\mathcal{D}}_{\mathcal{P}}$ to the formal neighborhood $\mathrm{FN}\left(\widetilde{\mathfrak{g}}_{\mathcal{P}}^{*}(1) \times_{\mathfrak{h}^{*(1)} / W_{\mathcal{P}}} W_{\mathcal{P}} \bullet \lambda\right)$, is equivalent to the pull-back of an Azumaya algebra on $\mathrm{FN}\left(\mathfrak{g}^{*(1)} \times_{\mathfrak{h}^{*(1)} / W} W \bullet\right.$ $\lambda)$.

In particular, for any $\chi$ with $(\chi, W \bullet \lambda) \in \mathcal{Z}(\mathrm{U})$, the restriction of $\widetilde{\mathcal{D}}_{\mathcal{P}}$ to the formal neighborhood of $\mathcal{P}_{\chi, \nu}{ }^{(1)}$ in $\mathcal{Z}\left(\widetilde{\mathcal{D}}_{\mathcal{P}}\right)$ is a split Azumaya algebra (here $\nu=\operatorname{AS}(\lambda)$ as above).

An equivalence and a splitting as above can be assigned in a canonical way to a choice of $\mu \in \Lambda$ such that $\lambda-d \mu \in \mathfrak{h}_{\mathrm{unr}}^{*}$.

The equivalence and the splitting are compatible with pull-backs from $\mathcal{Z}\left(\widetilde{\mathcal{D}}_{\mathcal{Q}}\right)$ to $\mathcal{Z}\left(\widetilde{\mathcal{D}}_{\mathcal{P}}\right)$ for a map of partial flag varieties $\mathcal{P} \rightarrow \mathcal{Q}$, and with replacing $\mu$ by $\mu^{\prime} \in \mu+p \Lambda$, as in Lemma 1.3.3. 
Remark 1.3.5. Assume that $\lambda$ is integral. Then a splitting bundle on the formal neighborhood of a parabolic Springer fiber can be constructed by a simpler method, which is completely parallel to the corresponding construction in $[\mathrm{BMR}]$.

Namely, if $\lambda \in \mathfrak{h}_{\mathbb{F}_{p}}^{*}$ is $\mathcal{P}$-unramified, then we have $\operatorname{Stab}_{W}(\lambda) \supset W_{\mathcal{P}}$. We can find a character $\eta \in \Lambda$ such that $d \eta=\lambda$ and $\operatorname{Stab}_{W}(\eta) \supset W_{\mathcal{P}}$. Each such $\eta$ defines a splitting bundle $\mathcal{M}=\mathcal{M}_{\chi, \eta}^{\mathcal{P}}$ as follows (cf. [BMR, Section 5]).

Consider first the case $\eta=-\rho$. Proposition 1.2.3.d shows that the restriction of the Azumaya algebra $\widetilde{\mathcal{D}}_{\mathcal{P}}$ to $\operatorname{FN}_{\mathcal{Z}(\widetilde{\mathcal{D}})}\left[\left(\varpi^{\mathcal{P}}\right)^{-1}(\chi,-\rho)\right] \cong$ $\left(\mathrm{FN}_{\widetilde{\mathfrak{g}}_{\mathcal{P}}}\left(\operatorname{pr}^{-1}(\chi, 0)\right)\right)^{(1)}$ is canonically isomorphic to the pull-back under $\varpi^{\mathcal{P}}$ of the Azumaya algebra $\mathrm{U}$ on $\mathrm{FN}_{\mathcal{Z}(\mathrm{U})}(\chi,-\rho)$. We fix a splitting bundle $\mathcal{M}_{\chi,-\rho}^{\mathcal{P}}$ of the form $\mathcal{M}_{\chi,-\rho}^{\mathcal{P}}=\left(\varpi^{\mathcal{P}}\right)^{*} \mathcal{M}_{0}$ where $\mathcal{M}_{0}$ is a splitting bundle for $\mathrm{U}$ on the formal neighborhood $\mathrm{FN}_{\mathcal{Z}(\mathrm{U})}(\chi,-\rho)$.

Let now $\eta \in \Lambda$ be any character as above. Then $\eta+\rho$ is a character of the Levi $L \subset P$, thus we have a line bundle $\mathcal{O}_{\mathcal{P}, \eta+\rho}$ on $\mathcal{P}$. We set $\mathcal{M}_{\chi, \eta}^{\mathcal{P}}=\mathcal{O}_{\mathcal{P}, \eta+\rho} \otimes_{\mathcal{O}_{\mathcal{P}}} \mathcal{M}_{\chi,-\rho}^{\mathcal{P}}$. In view of Lemma 1.2.1 this is indeed the required splitting bundle.

It is clear that given two partial flag varieties $\mathcal{P}=G / P \rightarrow \mathcal{Q}=G / Q$, the constructed splitting bundles enjoy the following compatibility:

$$
\left(\pi_{\mathcal{Q}}^{\mathcal{P}}\right)^{*} \mathcal{M}_{\chi, \nu}^{\mathcal{Q}} \cong \mathcal{M}_{\chi, \nu}^{\mathcal{P}}
$$

for any $\nu \in \Lambda$ such that $\operatorname{Stab}_{W}(\nu) \supset W_{\mathcal{Q}} \supset W_{\mathcal{P}}$. This isomorphism is compatible with the actions of $\widetilde{\mathcal{D}}_{\mathcal{P}}, \widetilde{\mathcal{D}}_{\mathcal{Q}}$ via the isomorphism (1).

We leave it as an exercise to the reader to check that the splitting bundles produced by this construction are also obtained by the construction of Corollary 1.3.4. This compatibility will not be used in the paper.

\subsection{Derived categories}

1.4.1. Derived categories of sheaves supported on a subscheme

Let $\mathcal{A}$ be a coherent sheaf on a Noetherian scheme $\mathfrak{X}$ equipped with an associative $\mathcal{O}_{\mathfrak{X}}$-algebra structure. We denote by $\bmod ^{c}(\mathcal{A})$ the abelian category of coherent sheaves of $\mathcal{A}$-modules. We also use notations $\mathcal{C}$ oh $(\mathfrak{X})$ if $\mathcal{A}=\mathcal{O}_{\mathfrak{X}}$ and $\bmod ^{f g}(\mathcal{A})$ if $\mathfrak{X}$ is affine.

For a subscheme $\mathfrak{Y}$ we denote by $\bmod _{\mathfrak{Y}}^{c}(\mathcal{A})$ the full subcategory of coherent $\mathcal{A}$-modules supported set-theoretically in $\mathfrak{Y}$, i.e., killed by some power of the ideal sheaf $\mathcal{I}_{\mathfrak{Y}}$. Then the tautological functor $\mathrm{D}^{b}\left[\bmod _{\mathfrak{Y}}^{c}(\mathcal{A})\right] \rightarrow$ 
$\mathrm{D}^{b}\left[\bmod ^{c}(\mathcal{A})\right]$ is a full embedding and the image consists of all $\mathcal{F} \in$ $\mathrm{D}^{b}\left[\bmod ^{c}(\mathcal{A})\right]$ that satisfy the equivalent conditions: i) $\mathcal{F}$ is killed by a power of the ideal sheaf $\mathcal{I}_{\mathfrak{Y}}$, i.e. the tautological arrow $\mathcal{I}_{\mathfrak{Y}}^{n} \otimes_{\mathcal{O}} \mathcal{F} \rightarrow \mathcal{F}$ is zero for some $n$; and ii) the cohomology sheaves of $\mathcal{F}$ lie in $\bmod _{\mathfrak{Y}}^{c}(\mathcal{A})$ (see, e.g., $[\mathrm{BMR}])$.

We will use this for the sheaves of algebras $\widetilde{\mathcal{D}}_{\mathcal{P}}$ and $U$ over $\widetilde{\mathfrak{g}}_{\mathcal{P}}^{*}(1)$ and $\mathfrak{g}^{*(1)}$ respectively (or $\mathcal{Z}\left(\widetilde{\mathcal{D}}_{\mathcal{P}}\right)$ and $\mathcal{Z}(\mathrm{U})$ respectively). For $\lambda \in \mathfrak{h}^{*}$ we have the abelian categories $\bmod ^{c}\left(\mathcal{D}_{\mathcal{P}}^{\lambda}\right) \subseteq \bmod _{\lambda}^{c}\left(\widetilde{\mathcal{D}}_{\mathcal{P}}\right) \subseteq \bmod ^{c}\left(\widetilde{\mathcal{D}}_{\mathcal{P}}\right)$; the second category is a particular case of the above situation where $\mathfrak{X}=\mathcal{Z}\left(\widetilde{\mathcal{D}}_{\mathcal{P}}\right)$ and $\mathfrak{Y}$ is the preimage of $\lambda \bmod W_{\mathcal{P}}$ under the projection $\mathcal{Z}\left(\widetilde{\mathcal{D}}_{\mathcal{P}}\right) \rightarrow \mathfrak{h}^{*} / W_{\mathcal{P}}$. We also have $\bmod ^{f g}\left(\mathrm{U}^{\lambda}\right) \subseteq \bmod _{\lambda}^{f g}(\mathrm{U}) \subseteq \bmod ^{f g}(\mathrm{U})$, where the second category is obtained as above for $\mathfrak{X}=\mathcal{Z}(U)$ and $\mathfrak{Y}$ being the preimage of $\lambda \bmod W$ under the projection to $\mathcal{Z}_{H C}$. For the corresponding triangulated categories we get $\mathrm{D}^{b}\left[\bmod ^{c}\left(\mathcal{D}_{\mathcal{P}}^{\lambda}\right)\right] \rightarrow \mathrm{D}^{b}\left[\bmod _{\lambda}^{c}\left(\widetilde{\mathcal{D}}_{\mathcal{P}}\right)\right] \subseteq \mathrm{D}^{b}\left[\bmod ^{c}\left(\widetilde{\mathcal{D}}_{\mathcal{P}}\right)\right]$, $\mathrm{D}^{b}\left[\bmod ^{f g}\left(\mathrm{U}^{\lambda}\right)\right] \rightarrow \mathrm{D}^{b}\left[\bmod _{\lambda}^{f g}(\mathrm{U})\right] \subseteq \mathrm{D}^{b}\left[\bmod ^{f g}(\mathrm{U})\right]$. We get similar categories for $(\chi, \lambda) \in \mathcal{Z}(\mathrm{U})$.

\subsubsection{The global section functors on $D$-modules}

We choose appropriate derived global section functors for $\bmod ^{c}\left(\mathcal{D}_{\mathcal{P}}^{\lambda}\right) \subseteq$ $\bmod _{\lambda}^{c}\left(\widetilde{\mathcal{D}}_{\mathcal{P}}\right) \subseteq \bmod ^{c}\left(\widetilde{\mathcal{D}}_{\mathcal{P}}\right)$. We start with the functor on quasi-coherent sheaves $\Gamma: \bmod ^{q c}\left(\mathcal{O}_{\mathcal{P}}\right) \rightarrow$ Vect. The map $\widetilde{U} \rightarrow \Gamma(\widetilde{\mathcal{D}})$ gives functors

$$
\begin{aligned}
& \bmod ^{q c}\left(\widetilde{\mathcal{D}}_{\mathcal{P}}\right) \stackrel{\Gamma_{\widetilde{\mathcal{D}}_{\mathcal{P}}}}{\longrightarrow} \bmod (\mathrm{U}), \quad \bmod _{\lambda}^{q c}\left(\widetilde{\mathcal{D}}_{\mathcal{P}}\right) \stackrel{\Gamma_{\widetilde{\mathcal{D}}_{\mathcal{P}}, \lambda}}{\longrightarrow} \bmod _{\lambda}(\mathrm{U}), \quad \text { and } \\
& \bmod ^{q c}\left(\mathcal{D}_{\mathcal{P}}^{\lambda}\right) \stackrel{\Gamma_{\mathcal{D}_{\mathcal{P}}^{\lambda}}}{\longrightarrow} \bmod \left(\mathrm{U}^{\lambda}\right) .
\end{aligned}
$$

We derive these functors in the above abelian categories (this can be done because the categories of modules have direct limits), and this gives

$$
\begin{aligned}
& \mathrm{R} \Gamma: \mathrm{D}\left[\bmod ^{q c}\left(\mathcal{O}_{\mathcal{P}}\right)\right] \longrightarrow \mathrm{D}[\text { Vect }], \\
& \mathrm{R} \Gamma_{\widetilde{\mathcal{D}}_{\mathcal{P}}}: \mathrm{D}\left[\bmod ^{q c}\left(\widetilde{\mathcal{D}}_{\mathcal{P}}\right)\right] \longrightarrow \mathrm{D}[\bmod (\mathrm{U})], \\
& \mathrm{R}_{\widetilde{\mathcal{D}}_{\mathcal{P}}, \lambda}: \mathrm{D}\left[\bmod _{\lambda}^{q c}\left(\widetilde{\mathcal{D}}_{\mathcal{P}}\right)\right] \longrightarrow \mathrm{D}\left[\bmod _{\lambda}(\mathrm{U})\right] \text {, } \\
& \mathrm{R}_{\mathcal{D}_{\mathcal{P}}^{\lambda}}: \mathrm{D}\left[\bmod ^{q c}\left(\mathcal{D}_{\mathcal{P}}^{\lambda}\right)\right] \longrightarrow \mathrm{D}\left[\bmod \left(\mathrm{U}^{\lambda}\right)\right] .
\end{aligned}
$$

The following properties were explained in [BMR, Section 3.1.9] for the particular case $\mathcal{P}=\mathcal{B}$; the same arguments apply in our present generality. 
(0) All of these functors have finite homological dimension, so they give functors between bounded derived categories.

(1) The derived functors commute with the forgetful functors, for instance $\operatorname{Forg}_{\mathbb{k}} \widetilde{\mathrm{U}} \circ \mathrm{R} \Gamma_{\widetilde{\mathcal{D}}} \cong \mathrm{R} \Gamma \circ \operatorname{Forg}_{\mathcal{O}}^{\widetilde{\mathcal{D}}}$ canonically for the forgetful functors $\operatorname{Forg}_{\mathcal{O}}^{\widetilde{\mathcal{D}}}: \bmod ^{q c}(\widetilde{\mathcal{D}}) \rightarrow \bmod ^{q c}(\mathcal{O}), \operatorname{Forg}_{\mathbb{k}} \widetilde{\operatorname{U}}: \bmod (\widetilde{\mathrm{U}}) \rightarrow \operatorname{Vect}_{\mathbb{k}}$

(2) The above (derived) functors of global sections preserve coherence, i.e. $R \Gamma_{\widetilde{\mathcal{D}}}$ sends the full subcategory $\mathrm{D}^{b}\left[\bmod ^{c}(\widetilde{\mathcal{D}})\right] \subset \mathrm{D}^{b}\left[\bmod ^{q c}(\widetilde{\mathcal{D}})\right]$ into the full subcategory $\mathrm{D}^{b}\left[\bmod ^{f g}(\widetilde{\mathrm{U}})\right] \subset \mathrm{D}^{b}[\bmod (\widetilde{\mathrm{U}})]$, etc.

\subsection{Equivalences}

The following Theorem is the main result of this section. We analyze the necessary conditions on $\lambda$ in Lemma 1.5.2. In particular, we find that for each Harish-Chandra character the corresponding category of U-modules has at least one localization, i.e., there is some $\lambda$ in the corresponding $W$ orbit in $\mathfrak{h}^{*}$, and some partial flag variety $\mathcal{P}$ such that all parts of the theorem below apply (Lemma 1.5.2.c).

We say that $\lambda$ is $\mathcal{P}$-regular if the stabilizer $(W, \bullet)_{\lambda}$ lies in $W_{\mathcal{P}}$. We say that a Harish-Chandra character has a singularity of $\mathcal{P}$-type if the corresponding $W$-orbit in $\mathfrak{h}^{*}$ contains $\lambda$ with the stabilizer $W_{\lambda}=W_{\mathcal{P}}$.

Theorem 1.5.1. Consider a $\mathcal{P}$-regular $\lambda \in \mathfrak{h}^{*}$.

(a) The global section functors provide equivalences of triangulated categories

$$
\begin{aligned}
& \mathrm{R}_{\widetilde{\mathcal{D}}_{\mathcal{P}}, \lambda}: \mathrm{D}^{b}\left[\bmod _{\lambda}^{c}\left(\widetilde{\mathcal{D}}_{\mathcal{P}}\right)\right] \cong \mathrm{D}^{b}\left[\bmod _{\lambda}^{f g}(\mathrm{U})\right] . \\
& \mathrm{R}_{\mathcal{D}_{\mathcal{P}}^{\lambda}}: \mathrm{D}^{b}\left[\bmod ^{c}\left(\mathcal{D}_{\mathcal{P}}^{\lambda}\right)\right] \stackrel{\cong}{\longrightarrow} \mathrm{D}^{b}\left[\bmod ^{f g}\left(\mathrm{U}^{\lambda}\right)\right] .
\end{aligned}
$$

(b) For any $\chi \in \mathfrak{g}^{*(1)}$, compatible with $\lambda($ i.e., $(\chi, \lambda) \in \mathcal{Z}(\mathrm{U}))$, the above equivalences restrict to equivalences of full subcategories with a generalized Frobenius character $\chi$

$$
\begin{aligned}
\mathrm{D}^{b}\left[\bmod _{\lambda, \chi}^{c}\left(\widetilde{\mathcal{D}}_{\mathcal{P}}\right)\right] & \cong \mathrm{D}^{b}\left[\bmod _{\lambda, \chi}^{f g}(\mathrm{U})\right] \quad \text { and } \\
\mathrm{D}^{b}\left[\bmod _{\chi}^{c}\left(\mathcal{D}_{\mathcal{P}}^{\lambda}\right)\right] & \cong \mathrm{D}^{b}\left[\bmod _{\chi}^{f g}\left(\mathrm{U}^{\lambda}\right)\right] .
\end{aligned}
$$

(c) If $\lambda$ is also a $\mathcal{P}$-unramified weight, then there are equivalences (set 


$$
\begin{aligned}
&\nu=\operatorname{AS}(\lambda))(10) \\
& \mathrm{D}^{b}\left[\bmod _{(\lambda, \chi)}^{f g}(\mathrm{U})\right] \cong \mathrm{D}^{b}\left[\bmod _{(\lambda, \chi)}^{c}\left(\widetilde{\mathcal{D}}_{\mathcal{P}}\right)\right] \cong \mathrm{D}^{b}\left[\mathcal{C}_{\left.o h_{\mathcal{P}_{\chi, \nu}(1)}\left(\widetilde{\mathfrak{g}}_{\mathcal{P}}^{*}{ }^{(1)}\right)\right]}\right. \\
& \mathrm{D}^{b}\left[\bmod _{\chi}^{f g}\left(\mathrm{U}^{\lambda}\right)\right] \cong \mathrm{D}^{b}\left[\bmod _{\chi}^{c}\left(\mathcal{D}_{\mathcal{P}}^{\lambda}\right)\right] \cong \mathrm{D}^{b}\left[\mathcal{C}_{o h_{\mathcal{P}_{\chi, \nu}}{ }^{(1)}}\left(\widetilde{\mathfrak{g}}_{\mathcal{P}}^{*}{ }^{(1)} \times_{\mathfrak{h}^{*(1)}} \nu\right)\right] .
\end{aligned}
$$

The proof will be given in Section 1.9.

Remark. It would be interesting to describe explicitly objects in $\mathrm{D}^{b}\left[\operatorname{Coh}_{\mathcal{P}_{\chi, \nu}(1)}\left(\widetilde{\mathfrak{g}}_{\mathcal{P}}^{*}{ }^{(1)} \times_{\mathfrak{h}^{*}(1)} \nu\right)\right]$ corresponding to (at least some) irreducible $\mathrm{U}$ modules, and also the locally free sheaves on the formal neighborhood of $\mathcal{P}_{\chi, \nu}{ }^{(1)}$ corresponding to indecomposable projective pro-objects in $\bmod _{\chi}^{f g}\left(\mathrm{U}^{\lambda}\right), \bmod _{(\lambda, \chi)}^{f g}(\mathrm{U})$. For $\mathcal{P}=\mathcal{B}$ this has been done in a few cases in [BMR, 5.3.3]. More examples can be computed using Lemma and Remark 2.2.5, or Remark 1.10.8 below.

LEMMA 1.5.2. (a) All parts of the theorem apply precisely when $\lambda$ is $\mathcal{P}$-regular and $\mathcal{P}$-unramified. This is equivalent to the following relation of stabilizers:

$$
W_{\lambda}=W_{\mathcal{P}} \cap W_{\operatorname{AS}(\lambda)} .
$$

(b) A sufficient condition is given by $W_{\lambda}=W_{\mathcal{P}}$. For integral $\lambda$ this is an equivalent condition.

(c) Each $W$-orbit in $\mathfrak{h}^{*}$ contains $\lambda$ such that the singularity of $\lambda$ is of type $\mathcal{P}$ for some partial flag variety $\mathcal{P}$, i.e., $W_{\lambda}=W_{\mathcal{P}}$.

(d) If $\lambda$ is the differential of a character $\nu \in \Lambda$ that lies in the closure of the fundamental alcove $A_{0}$, but not on any face corresponding to an affine coroot $\check{\alpha} \in I_{\text {aff }} \backslash I$, then $W_{\lambda}=W_{\mathcal{P}}$ is satisfied when $\mathcal{P}$ is the partial flag variety corresponding to the set of hyperplanes that contain $\nu$.

Proof. (a) is clear from definitions since $\lambda$ is $\mathcal{P}$-regular if $(W, \bullet)_{\lambda} \subseteq W_{\mathcal{P}}$, and $\mathcal{P}$-unramified if $\left(W_{\mathcal{P}}, \bullet\right)_{\lambda}=\left(W_{\mathcal{P}}\right)_{\mathrm{AS}(\lambda)}$, i.e., $(W, \bullet)_{\lambda} \cap W_{\mathcal{P}}=W_{\mathrm{AS}(\lambda)} \cap$ $W_{\mathcal{P}}$.

(b) The first claim follows from $W_{\lambda} \subseteq W_{\mathrm{AS}(\lambda)}$. For an integral weight $\lambda \in \mathfrak{h}_{\mathbb{F}_{p}}^{*}$ the stabilizer of $\operatorname{AS}(\lambda)=0$ is $W$, so the condition in (a) reduces to $W_{\lambda}=W_{\mathcal{P}}$.

(c) follows from Lemma 1.1.3.

\footnotetext{
${ }^{10}$ The equivalences depend on the choice of the splitting bundle for $U$ on the formal neighborhood of the point $(\chi, \lambda)$ in $\mathcal{Z}(\mathrm{U})$.
} 
(d) For any character $\nu$ the quotient map $W_{\text {aff }}^{\prime} \rightarrow W$ gives an isomorphism of stabilizers $\left(W_{\text {aff }}^{\prime}, \bullet\right)_{\nu} \stackrel{\cong}{\longrightarrow}(W, \bullet)_{d \nu}$. Because of the assumption on $p$ one has $\left(W_{\text {aff }}, \bullet\right)_{\nu}=\left(W_{\text {aff }}^{\prime}, \bullet\right)_{\nu}$. Finally, by Chevalley's theorem $\left(W_{\text {aff }}, \bullet\right)_{\nu}=W_{\mathcal{P}}$. Now, the inclusion $W_{\mathcal{P}} \subseteq(W, \bullet)_{d \nu}$ is equality since the orders of groups are the same.

Corollary. If in the conditions of Theorem 1.5.1 the weight $\lambda$ is $\mathcal{P}$ regular and $\mathcal{P}$-unramified, then there is a natural isomorphism of Grothendieck groups $K\left(\mathrm{U}_{\chi}^{\lambda}\right) \cong K\left(\mathcal{P}_{\chi, \nu}\right)$. In particular, the number of irreducible $\mathrm{U}_{\chi}^{\lambda}$-modules is the rank of $K\left(\mathcal{P}_{\chi, \nu}\right)$.

Remark. The method of [BMR, Section 7] can be used to show that for large $p$ the rank of $K\left(\mathcal{P}_{\chi, \nu}\right)$ equals the sum of Betti numbers of the corresponding parabolic Springer fiber $\mathcal{P}_{\chi^{\prime}, \nu^{\prime}}$ in characteristic zero; here $\nu^{\prime}$ is an element in the Lie algebra over a characteristic zero field whose centralizer is the Levi subgroup of the same type as the centralizer of $\nu$, and $\chi^{\prime}$ corresponds to $\chi$ under the bijection arising from the Bala-Carter classification.

\subsubsection{Reduction of the theorem to the part (a)}

A proof of the part (a) will be given in Section 1.9. Here we assume (a) and prove (b) and (c).

(b) $\mathcal{O}\left(\mathfrak{g}^{*(1)}\right)$ acts on the categories $\bmod ^{c}\left(\widetilde{\mathcal{D}}_{\mathcal{P}}\right), \bmod ^{f g}(\mathrm{U})$ etc., and on their derived categories. This means an algebra homomorphism from $\mathcal{O}\left(\mathfrak{g}^{*(1)}\right)$ to the algebra of natural endomorphisms of the identity functor (often called the center of the category). The homomorphism takes $f \in \mathcal{O}\left(\mathfrak{g}^{*(1)}\right)$ into a natural transformation defined by $m \mapsto f m$ for an object $M$ and $m \in M$. The equivalences in (a) are equivariant under $\mathcal{O}\left(\mathfrak{g}^{*(1)}\right)$ and therefore they restrict to the full subcategories of objects on which the Frobenius center acts by the generalized character $\chi$ (see 1.4.1).

(c) Consider $\widetilde{\mathcal{D}}_{\mathcal{P}}$-modules as sheaves on $\mathcal{Z}\left(\widetilde{\mathcal{D}}_{\mathcal{P}}\right)$. They have a generalized central character $(\chi, \lambda)$ precisely if they are set theoretically supported on $\mathcal{Z}\left(\widetilde{\mathcal{D}}_{\mathcal{P}}\right) \times_{\mathcal{Z}(\mathrm{U})}(\chi, \lambda)=\mathcal{P}_{\chi, \nu}^{(1)}$. Since $\lambda$ is $\mathcal{P}$-unramified, the restriction of $\widetilde{\mathcal{D}}_{\mathcal{P}}$ to the formal neighborhood of the Springer fiber $\mathcal{P}_{\chi, \nu}{ }^{(1)}$ in $\mathcal{Z}\left(\widetilde{\mathcal{D}}_{\mathcal{P}}\right)$ (the same as the formal neighborhood $\mathrm{FN}_{\mathcal{P}_{\chi, \nu}}\left(\widetilde{\mathfrak{g}}_{\mathcal{P}}^{*}\right)^{(1)}$ of $\mathcal{P}_{\chi, \nu}{ }^{(1)}$ in $\left.\widetilde{\mathfrak{g}}_{\mathcal{P}}^{*}{ }^{(1)}\right)$, is a trivial Azumaya algebra by the Corollary 1.3.4. Therefore any choice of a splitting vector bundle $\mathcal{M}_{\chi, \lambda}^{\mathcal{P}}$ on this formal neighborhood provides 
equivalences of abelian categories

$$
\operatorname{Coh}_{\mathcal{P}_{\chi, \nu}(1)}\left(\widetilde{\mathfrak{g}}_{\mathcal{P}}^{*}{ }^{(1)}\right) \stackrel{\cong}{\longrightarrow} \operatorname{Coh}_{\mathcal{P}_{\chi, \nu}{ }^{(1)}}\left(\mathcal{Z}\left(\widetilde{\mathcal{D}}_{\mathcal{P}}\right)\right) \stackrel{\mathcal{M}_{\chi, \lambda}^{\mathcal{P}} \otimes-}{\cong} \bmod _{\chi, \lambda}^{c}\left(\widetilde{\mathcal{D}}_{\mathcal{P}}\right)
$$

This proves the first claim. We get the second one similarly, as $\mathcal{D}_{\mathcal{P}}^{\lambda}$-modules are sheaves on $\mathcal{Z}\left(\widetilde{\mathcal{D}}_{\mathcal{P}}\right) \times_{\mathfrak{h}^{*} / W_{\mathcal{P}}} \lambda$.

\subsection{Localization as the left adjoint of global sections}

1.6.1. Localization functors

We start with the localization functor $\operatorname{Loc}_{\mathcal{P}}$ from finitely generated U-modules to coherent $\widetilde{\mathcal{D}}_{\mathcal{P}}$ modules,

$$
\operatorname{Loc}_{\mathcal{P}}(M) \stackrel{\text { def }}{=} \widetilde{\mathcal{D}}_{\mathcal{P}} \otimes \mathrm{U} M
$$

For each $\lambda \in \mathfrak{h}^{*}$ it restricts to a functor

$$
\operatorname{Loc}_{\mathcal{P}}^{\lambda}: \bmod ^{f g}\left(\mathrm{U}^{\lambda}\right) \longrightarrow \bmod ^{c}\left(\mathcal{D}_{\mathcal{P}}^{\lambda}\right), \quad \operatorname{Loc}_{\mathcal{P}}^{\lambda}(M) \stackrel{\text { def }}{=} \mathcal{D}_{\mathcal{P}}^{\lambda} \otimes_{\mathrm{U}^{\lambda}} M
$$

Since $U$ has finite homological dimension, the functor $\operatorname{Loc}_{\mathcal{P}}$ has a left derived functor $\mathrm{D}^{b}\left[\bmod ^{f g}(\mathrm{U})\right] \stackrel{\mathcal{L}_{\mathcal{P}}}{\longrightarrow} \mathrm{D}^{b}\left[\bmod ^{c}\left(\widetilde{\mathcal{D}}_{\mathcal{P}}\right)\right]$. Fix $\lambda \in \mathfrak{h}^{*}$, for any $M \in \mathrm{D}^{b}\left[\bmod _{\lambda}^{f g}(\mathrm{U})\right]$ the action of $\mathfrak{Z}_{\mathrm{HC}}=S(\mathfrak{h})^{W}$ on $\mathcal{L}_{\mathcal{P}}(M)$ factors through an ideal $I$, the power of the maximal ideal corresponding to the orbit $W \bullet \lambda$. The finite-dimensional algebra $S(\mathfrak{h})^{W_{\mathcal{P}}} / I S(\mathfrak{h})^{W_{\mathcal{P}}}$, which also acts on $\mathcal{L}_{\mathcal{P}}(M)$, has a primitive idempotent for each orbit $W_{\mathcal{P}} \bullet \mu \subseteq W \bullet \lambda$. These primitive idempotents give a canonical decomposition $\mathcal{L}_{\mathcal{P}}(M)=$ $\bigoplus_{W_{\mathcal{P}} \bullet \mu \subseteq W \bullet \lambda} \mathcal{L}_{\mathcal{P}}^{W \bullet \lambda \rightarrow W_{\mathcal{P}} \bullet \mu}(M) \quad$ with $\quad \mathcal{L}_{\mathcal{P}}^{W \bullet \lambda \rightarrow W_{\mathcal{P}} \bullet \mu}(M) \in \mathrm{D}^{b}\left[\bmod _{\mu}^{c}\left(\widetilde{\mathcal{D}}_{\mathcal{P}}\right)\right]$. Localization with the generalized character $\lambda$ is the functor $\mathcal{L}_{\mathcal{P}} \stackrel{\text { def }}{=}$ $\mathcal{L}_{\mathcal{P}}^{W \bullet \lambda \rightarrow W_{\mathcal{P}} \bullet \lambda}: \mathrm{D}^{b}\left[\bmod _{\lambda}^{f g}(\mathrm{U})\right] \rightarrow \mathrm{D}^{b}\left[\bmod _{\lambda}^{c}\left(\widetilde{\mathcal{D}}_{\mathcal{P}}\right)\right]$

The functor $\operatorname{Loc}_{\mathcal{P}}^{\lambda}$ also has a left derived functor $\mathcal{L}_{\mathcal{P}}^{\lambda}: \mathrm{D}^{-}\left(\bmod ^{f g}\left(\mathrm{U}^{\lambda}\right)\right) \rightarrow$ $\mathrm{D}^{-}\left(\bmod ^{c}\left(\mathcal{D}_{\mathcal{P}}^{\lambda}\right)\right), \mathcal{L}_{\mathcal{P}}^{\lambda}(M)=\mathcal{D}_{\mathcal{P}}^{\lambda}{\stackrel{\otimes}{\mathrm{U}^{\lambda}}}^{L} M$. The algebra $\mathrm{U}^{\lambda}$ may have infinite homological dimension ${ }^{(11)}$ so a'priori $\mathcal{L}_{\mathcal{P}}^{\lambda}$ need not preserve the bounded derived categories.

LEMmA 1.6.2. (a) The functor $\mathcal{L}_{\mathcal{P}}$ is left adjoint to $\mathrm{R} \Gamma_{\widetilde{\mathcal{D}}_{\mathcal{P}}}$.

\footnotetext{
${ }^{11}$ For regular $\lambda$ finiteness of homological dimension follows from Theorem 1.5.1. For singular $\lambda$ the homological dimension is infinite. For instance, for $\lambda=-\rho$ the algebra $\mathrm{U}^{\lambda}$ is a split Azumaya algebra over the Frobenius twisted nilpotent cone.
} 
(b) The functor $\mathcal{L}_{\mathcal{P}}^{\widehat{\lambda}}$ is left adjoint to $\mathrm{R} \Gamma_{\widetilde{\mathcal{D}}_{\mathcal{P}}, \lambda}$.

(c) The functor $\mathcal{L}_{\mathcal{P}}^{\lambda}$ is left adjoint to the functor $\mathrm{D}^{-}\left(\bmod ^{c}\left(\mathcal{D}_{\mathcal{P}}^{\lambda}\right)\right) \stackrel{\mathrm{R} \Gamma_{\mathcal{D}}^{\lambda}}{\longrightarrow}$ $\mathrm{D}^{-}\left(\bmod ^{f g}\left(\mathrm{U}^{\lambda}\right)\right)$.

(d) For $\mathcal{P}$-regular $\lambda$ the localizations at $\lambda$ and at the generalized character $\lambda$ are compatible, i.e., for the obvious functors $\mathrm{D}^{-}\left(\bmod ^{f g}\left(\mathrm{U}^{\lambda}\right)\right) \stackrel{i}{\rightarrow}$ $\mathrm{D}^{-}\left(\bmod _{\lambda}^{f g}(\mathrm{U})\right)$ and $\mathrm{D}^{-}\left(\bmod ^{c}\left(\mathcal{D}_{\mathcal{P}}^{\lambda}\right)\right) \stackrel{\iota}{\rightarrow} \mathrm{D}^{-}\left(\bmod _{\lambda}^{c}\left(\widetilde{\mathcal{D}}_{\mathcal{P}}\right)\right)$, there is a canonical isomorphism $\iota \circ \mathcal{L}^{\lambda} \cong \mathcal{L}^{\widehat{\lambda}} \circ i$. This isomorphism is compatible with the adjunction arrows, i.e. for $M \in \mathrm{D}^{-}\left(\bmod ^{f g}\left(\mathrm{U}^{\lambda}\right)\right), \mathcal{F} \in \mathrm{D}^{-}\left(\bmod ^{c}\left(\mathcal{D}_{\mathcal{P}}^{\lambda}\right)\right)$ the composition:

$$
\begin{aligned}
\operatorname{Hom}\left(M, R \Gamma_{\lambda} \mathcal{F}\right) \cong \operatorname{Hom}\left(\mathcal{L}^{\lambda} M, \mathcal{F}\right) & \longrightarrow \operatorname{Hom}\left(\iota \mathcal{L}^{\lambda} M, \iota \mathcal{F}\right) \cong \operatorname{Hom}\left(\mathcal{L}^{\widehat{\lambda}} i M, \iota \mathcal{F}\right) \\
& \cong \operatorname{Hom}\left(i M, R \Gamma_{\widehat{\lambda}} \iota \mathcal{F}\right) \cong \operatorname{Hom}\left(i M, i R \Gamma_{\lambda} \mathcal{F}\right)
\end{aligned}
$$

coincides with the map induced by functoriality of $i$.

Proof. (a) One checks from the definitions that the functors between abelian categories form adjoint pairs. Since $\bmod ^{q c}\left(\widetilde{\mathcal{D}}_{\mathcal{P}}\right)$ (respectively, $\bmod (\mathrm{U}))$ has enough injective (respectively, projective) objects, and the functors $\Gamma_{\mathcal{P}}, \operatorname{Loc}_{\mathcal{P}}$ have bounded homological dimension, it follows that their derived functors form an adjoint pair of functors between bounded derived categories. This adjunction restricts to the required adjunction for $\mathcal{L}_{\mathcal{P}}$ and $\mathrm{R} \Gamma_{\widetilde{\mathcal{D}}_{\mathcal{P}}}$.

(b) follows from (a) by passing to summands.

(c) The proof is analogous to the proof for the first pair.

(d) For a module $M$ with character $\lambda$, the $S \mathfrak{h}$-module $\mathcal{L}_{\mathcal{P}} M$ is supported on the subscheme $\mathfrak{h}^{*} / W_{\mathcal{P}} \times_{\mathfrak{h}^{*} / W} \lambda \subseteq \mathfrak{h}^{*}$. Since $\lambda$ is $\mathcal{P}$-regular the map $\mathfrak{h}^{*} / W_{\mathcal{P}} \rightarrow \mathfrak{h}^{*} / W$ is unramified at $W_{\mathcal{P}} \bullet \lambda$. Then the point $\lambda$ is a connected component of $\mathfrak{h}^{*} / W_{\mathcal{P}} \times_{\mathfrak{h}^{*} / W} \lambda$, and the corresponding summand of $\mathcal{L}_{\mathcal{P}} M$ is $\operatorname{Loc}_{\mathcal{P}}^{\lambda} M$.

Corollary 1.6.3. If $\lambda$ is $\mathcal{P}$-regular the functor $\mathcal{L}_{\mathcal{P}}^{\lambda}$ sends the bounded derived category $\mathrm{D}^{b}\left[\bmod ^{f g}\left(\mathrm{U}^{\lambda}\right)\right]$ to $\mathrm{D}^{b}\left[\bmod ^{c}\left(\mathcal{D}_{\mathcal{P}}^{\lambda}\right)\right]$.

\subsubsection{Localization is fully faithful}

This is one way to view the following claim (if a functor $L$ between triangulated categories has a right adjoint $R$ then $L$ is fully faithful if and only if the map id $\rightarrow R \circ L$ is an isomorphism). 
Proposition. (a) The composition $\mathrm{R}_{\widetilde{\mathcal{D}}_{\mathcal{P}}} \circ \mathcal{L}_{\mathcal{P}}: \mathrm{D}^{b}\left[\bmod ^{f g}(\mathrm{U})\right] \rightarrow$ $\mathrm{D}^{b}\left[\bmod ^{f g}(\mathrm{U})\right]$ is isomorphic to the functor $M \mapsto M \otimes_{S(\mathfrak{h})^{W}} S(\mathfrak{h})^{W_{\mathcal{P}}}$.

(b) For P-regular $\lambda$ the adjunction map id $\rightarrow \mathrm{R} \Gamma_{\widetilde{\mathcal{D}}_{\mathcal{P}, \lambda}} \circ \mathcal{L}^{\widehat{\lambda}}$ is an isomorphism on $\mathrm{D}^{b}\left[\bmod _{\lambda}^{f g}(\mathrm{U})\right]$.

(c) For any $\lambda$, the adjunction map is an isomorphism id $\rightarrow \mathrm{R} \Gamma_{\mathcal{D}_{\mathcal{P}}^{\lambda}} \circ \mathcal{L}_{\mathcal{P}}^{\lambda}$ on $\mathrm{D}^{-}\left(\bmod ^{f g}\left(\mathrm{U}^{\lambda}\right)\right)$.

Proof. (a) For any U-module $M$ the action of $\mathrm{U}$ on $\Gamma_{\widetilde{\mathcal{D}}_{\mathcal{P}}}\left(\mathcal{L}_{\mathcal{P}}(M)\right)$ extends to the action of $\Gamma\left(\widetilde{\mathcal{D}}_{\mathcal{P}}\right)=\widetilde{\mathrm{U}}^{W_{\mathcal{P}}}$. So, the adjunction map $M \rightarrow$ $\Gamma_{\widetilde{\mathcal{D}}_{\mathcal{P}}}\left(\mathcal{L}_{\mathcal{P}}(M)\right)$ extends to $S(\mathfrak{h})^{W_{\mathcal{P}}} \otimes_{S(\mathfrak{h})^{W}} M=\widetilde{\mathrm{U}}^{W_{\mathcal{P}}} \otimes_{\mathrm{U}} M \rightarrow \Gamma_{\widetilde{\mathcal{D}}_{\mathcal{P}}} \circ \mathcal{L}_{\mathcal{P}}(M)$. Proposition 1.2.3.b implies that if $M$ is a free module then this map is an isomorphism, while higher derived functors $\mathrm{R}^{i} \Gamma_{\widetilde{\mathcal{D}}_{\mathcal{P}}}\left(\mathcal{L}_{\mathcal{P}}(M)\right), i>0$, vanish. This yields (a). Statement (c) is proved in the same way using the second claim in Proposition 1.2.3.b.

To deduce (b) observe that for a $\mathcal{P}$-regular $\lambda$ and $M \in \mathrm{D}^{b}\left[\bmod _{\lambda}^{f g}(\mathrm{U})\right]$, we have a canonical decomposition of $M \otimes_{S(\mathfrak{h})^{W}} S(\mathfrak{h})^{W_{\mathcal{P}}}$ into summands $M \otimes_{\mathbb{k}} \mathcal{O}(C)$ over connected components $C$ of $\mathfrak{h}^{*} / W_{\mathcal{P}} \times \mathfrak{h}^{*} / W \lambda$. Since $\lambda$ is $\mathcal{P}$-regular, one such component is $\lambda$. The corresponding component of the adjunction morphism viewed as a map $M \rightarrow \bigoplus_{C} M \otimes_{\mathbb{k}} \mathcal{O}(C)$ is $\operatorname{id}_{M}$. Now the claim follows since the corresponding summand can be viewed as $\mathrm{R} \Gamma_{\widetilde{\mathcal{D}}_{\mathcal{P}, \lambda}}\left(\mathcal{L}_{\mathcal{P}}^{\widehat{\lambda}}(M)\right)$.

\subsection{Calabi-Yau categories}

The remaining ingredient of the proof of the localization theorem is the use of properties of the Calabi-Yau subclass of triangulated categories. These we recall here, for more detail see $[\mathrm{BMR}],[\mathrm{BK}]$ or the original paper [BKR].

\subsubsection{Relative Serre functors}

Let $\mathcal{O}$ be a finite type commutative algebra over a field; and let $D$ be an $\mathcal{O}$-linear triangulated category. A structure of an $\mathcal{O}$-triangulated category on $D$ is a functor $\mathrm{RHom}_{D / \mathcal{O}}: D^{o p} \times D \rightarrow \mathrm{D}^{b}\left[\bmod ^{f g}(\mathcal{O})\right]$, together with a functorial isomorphism $\operatorname{Hom}_{D}(X, Y) \cong \mathrm{H}^{0}\left(\operatorname{RHom}_{D / \mathcal{O}}(X, Y)\right)$. By an $\mathcal{O}$-Serre functor on $D$ we will mean an auto-equivalence $S: D \rightarrow D$ together with a natural (functorial) isomorphism $\mathbb{D}_{\mathcal{O}}\left[\operatorname{RHom}_{D / \mathcal{O}}(X, Y)\right] \cong$ $\operatorname{RHom}_{D / \mathcal{O}}(Y, S X)$ for all $X, Y \in D$. $\left(\mathbb{D}_{\mathcal{O}}\right.$ denotes the Grothendieck duality for $\mathcal{O}$-modules.) An $\mathcal{O}$-triangulated category will be called Calabi-Yau if for 
some $n \in \mathbb{Z}$ the shift functor $X \mapsto X[n]$ admits a structure of an $\mathcal{O}$-Serre functor.

1.7.2. Calabi-Yau categories have no retracts

The following lemma appears in our first paper [BMR, Lemma 3.5.2], a similar argument has appeared earlier [BKR, Theorem 2.3]. One can summarize it by: a retract of a Calabi-Yau category is a summand.

Lemma. Let $D$ be a Calabi-Yau $\mathcal{O}$-triangulated category for some commutative finitely generated algebra $\mathcal{O}$. Let $C$ be a triangulated category. Then a sufficient condition for a triangulated functor $L: C \rightarrow D$ to be an equivalence is given by

(i) L has a right adjoint functor $R$ and the adjunction morphism id $\rightarrow$ $R \circ L$ is an isomorphism, and

(ii) $R$ is not zero on any summand of $D$.

Proof. (i) implies that $L$ is a full embedding, i.e., we can consider $C$ as a full subcategory of $D$. Then id $\cong R \circ L$ can be thought of as a retraction from $D$ to $C$, however a retract of a Calabi-Yau category is a summand: the retract structure shows that any $d \in D$ is in an exact triangle $c \rightarrow d \rightarrow c^{\prime}$ with $c \in C$ and $c^{\prime} \in C^{\perp}$. Recall that $C^{\perp}$ is a full subcategory of $D$ with objects $x$ such that $\operatorname{Hom}_{D}(c, x)=0$ for all $c \in C$. Similarly, ${ }^{\perp} C$ is a full subcategory of $D$ with objects $x$ such that $\operatorname{Hom}_{D}(x, c)=0$ for all $c \in C$. Now the Calabi-Yau property exchanges left and right $C^{\perp}={ }^{\perp} C$, and then $D \cong C \oplus C^{\perp}$. Now (ii) implies $C^{\perp}=0$.

Another useful simple fact is the following lemma, which has been known for $\mathcal{A}=\mathcal{O}$ [BKR, Lemma 4.2].

LEMMA 1.7.3. Let $X$ be a connected scheme quasiprojective over a field $\mathbb{k}$, and let $\mathcal{A}$ be an algebra vector bundle over $X$ which is generically Azumaya. Then the category $\mathrm{D}^{b}\left[\bmod ^{c}(\mathcal{A})\right]$ is indecomposable. Moreover, if $Y \subset X$ is a connected closed subset then $\mathrm{D}^{b}\left[\bmod _{Y}^{c}(X, \mathcal{A})\right]$ is indecomposable.

Proof. This is already proved when $\mathcal{A}$ is Azumaya [BMR, Lemma 3.5.3]. The proof is valid in this more general case. 


\subsection{Frobenius algebras}

The main goal here is to prove that $\mathrm{D}^{b}\left[\bmod ^{c}(\mathrm{U})\right]$ is Calabi-Yau over $\mathfrak{g}^{*(1)}$ (see 1.8.2.c). A Frobenius algebra structure on a locally free algebra sheaf $\mathcal{A}$ over a scheme $X$ is a functional $\tau \in \mathcal{H} \mathrm{m}_{\mathcal{O}_{X}}\left(\mathcal{A}, \mathcal{O}_{X}\right)$ which is nondegenerate in the sense that the map $\widetilde{\tau}: \mathcal{A} \rightarrow \mathcal{H}_{\mathrm{om}_{\mathcal{O}_{X}}}\left(\mathcal{A}, \mathcal{O}_{X}\right),(\widetilde{\tau} a) b=\tau(a b)$, is an isomorphism ${ }^{(12)}$. We say that $\mathcal{A}$ is Frobenius if it has a Frobenius structure. We say that a map of schemes $X \stackrel{f}{\rightarrow} Y$ is Frobenius if it is finite and $f_{*} \mathcal{O}_{X}$ is a Frobenius algebra over $\mathcal{O}_{\mathcal{Y}}$.

Lemma 1.8.1. (a) Let $\mathcal{A}$ be a sheaf of algebras over a normal variety $X$ which is locally free of finite rank over $\mathcal{O}_{X}$. If $\mathcal{A}$ is Frobenius on an open set with a complement of codimension at least two (on each component), then $\mathcal{A}$ is Frobenius everywhere.

(b) Let $\mathcal{O}$ be a finite type commutative algebra over $\mathbb{k}$. Let $Y$ be a variety over $\mathbb{k}$ equipped with a projective morphism $\pi: Y \rightarrow \operatorname{Spec}(\mathcal{O})$ then $D=\mathrm{D}^{b}\left[\mathcal{C o h}_{Y}\right]$ is $\mathcal{O}$-triangulated by $\operatorname{RHom}_{D / \mathcal{O}}(\mathcal{F}, \mathcal{G}) \stackrel{\text { def }}{=} \mathrm{R} \pi_{*} \operatorname{RHom}(\mathcal{F}, \mathcal{G})$. If $Y$ is also smooth and quasiprojective, then for any Frobenius algebra $(\mathcal{B}, \tau)$ on $Y, \mathrm{D}^{b}\left[\bmod ^{c}(\mathcal{B})\right]$ has a natural structure of an $\mathcal{O}$-triangulated category, and the functor $\mathcal{F} \mapsto \mathcal{F} \otimes \omega_{Y}[\operatorname{dim} Y]$ has a natural structure of an $\mathcal{O}$-Serre functor. In particular, if $Y$ is a Calabi-Yau manifold (i.e., $\left.\omega_{Y} \cong \mathcal{O}_{Y}\right)$ then the $\mathcal{O}$-triangulated category $\mathrm{D}^{b}\left[\bmod ^{c}(\mathcal{B})\right]$ is Calabi-Yau.

(c) Any Azumaya algebra $\mathcal{C}$ over a scheme $Z$ has a canonical Frobenius structure given by the reduced trace.

(d) Let $X \stackrel{\pi}{\rightarrow} Y$ be a finite flat map of smooth varieties with trivial canonical classes. For any $\mathcal{O}_{X}$-algebra $\mathcal{A}$ there is a canonical bijection between the set of Frobenius structures on $\mathcal{A}$ over $X$ and the set of Frobenius structures on $\pi_{*} \mathcal{A}$ over $Y$.

(e) Any finite map of smooth varieties with trivial canonical classes $X \stackrel{f}{\rightarrow} Y$ has a canonical Frobenius structure.

Proof. (a) Restriction of $\mathcal{A}$ to an open $X_{0} \subseteq X$ with the complement of codimension $\geq 2$, carries a non-degenerate Frobenius functional $\tau_{0}$. Since $X$ is normal the section $\tau_{0}$ of $\mathcal{H}$ om $\left(\mathcal{A}, \mathcal{O}_{X}\right)$ on $X_{0}$ extends to $\mathcal{A} \stackrel{\tau}{\rightarrow} \mathcal{O}_{X}$ on $X$.

\footnotetext{
${ }^{12}$ Frobenius structures are the same as right $\mathcal{A}$-module isomorphisms $T: \mathcal{A} \stackrel{\cong}{\longrightarrow}$ $\mathcal{H}_{\mathcal{O}_{X}}\left(\mathcal{A}, \mathcal{O}_{X}\right)$ since such $T$ gives a map $\tau: \mathcal{A} \rightarrow \mathcal{O}_{X}$ via $\tau a=(T a) 1_{\mathcal{A}}$ such that $\widetilde{\tau}=T$.
} 
Since $\tau$ is non-degenerate outside of codimension two, it is nondegenerate on $X$.

(b) The first claim is obvious. To prove the second claim, let us suppose for a moment that $\mathcal{F}, \mathcal{G}$ are locally projective $\mathcal{B}$-modules of finite rank. The trace map $\mathcal{E}_{\operatorname{nd}_{\mathcal{B}}}(\mathcal{F}) \stackrel{\operatorname{tr}_{\mathcal{F}}}{\longrightarrow} \mathcal{B}$ leads to the pairing

$$
\mathcal{H}_{\mathcal{B}}(\mathcal{F}, \mathcal{G}) \otimes \mathcal{H}_{\operatorname{om}}(\mathcal{G}, \mathcal{F}) \longrightarrow \mathcal{E}_{\operatorname{nd}_{\mathcal{B}}}(\mathcal{F}) \stackrel{\tau \operatorname{otr}_{\mathcal{F}}}{\longrightarrow} \mathcal{O}_{Y}
$$

which is non-degenerate, i.e., it gives $\mathcal{H}_{\mathcal{B}}(\mathcal{G}, \mathcal{F}) \cong \mathcal{H}_{\mathcal{O}_{Y}}\left[\mathcal{H o m}_{\mathcal{B}}(\mathcal{F}, \mathcal{G})\right.$, $\left.\mathcal{O}_{Y}\right]$. From now on let $\mathcal{F}, \mathcal{G}$ be arbitrary objects of $\mathrm{D}^{b}\left[\bmod ^{c}(\mathcal{B})\right]$. Under our assumptions $\mathrm{D}^{b}[\mathcal{C}$ oh $(\mathcal{B})]$ is generated by locally projective modules. We conclude that there is an isomorphism $\operatorname{RH}_{\mathcal{B}}(\mathcal{G}, \mathcal{F}) \stackrel{\cong}{\longrightarrow} \operatorname{RHom}_{\mathcal{O}_{Y}}\left[\operatorname{RH}_{\mathcal{H}}(\mathcal{F}\right.$, $\left.\mathcal{G}), \mathcal{O}_{Y}\right]$ for any $\mathcal{F}, \mathcal{G} \in \mathrm{D}^{b}\left[\bmod ^{c}(\mathcal{B})\right]$. Since $\mathcal{D}_{Y} \cong \operatorname{RH}_{\mathcal{H}} \operatorname{mom}_{\mathcal{O}_{Y}}\left(-, \omega_{Y}[\operatorname{dim}(Y)]\right)$ for a smooth $Y$ we obtain

$$
\begin{aligned}
\mathbb{D}_{Y} \operatorname{RHom}_{\mathcal{B}}(\mathcal{F}, \mathcal{G}) & \left.\cong \operatorname{RH} \operatorname{com}_{\mathcal{O}_{Y}}\left[\operatorname{RHom}_{\mathcal{B}}(\mathcal{F}, \mathcal{G}), \mathcal{O}_{Y}\right)\right] \otimes \omega_{Y}[\operatorname{dim}(Y)] \\
& \cong \operatorname{RHom}_{\mathcal{B}}(\mathcal{G}, \mathcal{F}) \otimes \omega_{Y}[\operatorname{dim}(Y)] \\
& \cong \operatorname{RH}_{\mathcal{H}} \operatorname{om}_{\mathcal{B}}\left(\mathcal{G}, \mathcal{F} \otimes \omega_{Y}[\operatorname{dim}(Y)]\right)
\end{aligned}
$$

Finally, since Grothendieck-Serre duality commutes with proper direct images

$$
\begin{aligned}
\mathbb{D}_{\mathcal{O}}\left[\mathrm{R} \pi_{*} \operatorname{R\mathcal {H}}_{\mathcal{B}}(\mathcal{F}, \mathcal{G})\right] & \cong \mathrm{R} \pi_{*}\left[\mathbb{D}_{Y} \operatorname{R\mathcal {H}}_{\mathcal{B}}(\mathcal{F}, \mathcal{G})\right] \\
& \cong \mathrm{R} \pi_{*} \operatorname{RH} \mathcal{H o m}\left(\mathcal{G}, \mathcal{F} \otimes \omega_{Y}[\operatorname{dim} Y]\right) .
\end{aligned}
$$

(c) After an etale base change $\widetilde{Z} \rightarrow Z$, the algebra $\mathcal{C}$ is isomorphic to a matrix algebra $\mathcal{E}_{\operatorname{nd}_{\mathcal{O}_{\widetilde{Z}}}}(\mathcal{E})$ for some vector bundle $\mathcal{E}$. The trace $\mathcal{E}_{\operatorname{nd}_{\mathcal{O}_{\widetilde{Z}}}}(\mathcal{E}) \rightarrow$ $\mathcal{O}_{\widetilde{Z}}$ descends to the so called reduced trace $\mathcal{C} \rightarrow \mathcal{O}_{Z}$, which is clearly nondegenerate. (Actually all invariant polynomials descend.)

(d) Since the canonical classes of $X, Y$ are trivial, the GrothendieckSerre duality functor for $X, Y$ is given by $\mathcal{F} \mapsto \operatorname{RHom}(\mathcal{F}, \mathcal{O})[d]$ where $d=\operatorname{dim} X=\operatorname{dim} Y$ (we assume without loss of generality that $X$ and $Y$ are equidimensional). Since the Grothendieck-Serre duality commutes with proper, in particular, finite, push-forwards we have canonical isomorphisms

$$
\pi_{*} \mathcal{H}_{\mathrm{Om}_{X}}\left(\mathcal{A}, \mathcal{O}_{X}\right)=\mathcal{H}_{\mathcal{O}} \mathcal{O}_{X}\left(\pi_{*} \mathcal{A}, \mathcal{O}_{Y}\right)
$$

The isomorphism is compatible with (both left and right) $\pi_{*} \mathcal{A}$ action by functoriality of the isomorphism between the two compositions of proper 
push-forward and the Grothendieck-Serre duality. This gives a bijection of (iso)morphisms $\mathcal{A} \rightarrow \mathcal{H o m}_{\mathcal{O}_{X}}\left(\mathcal{A}, \mathcal{O}_{X}\right)$ and $\pi_{*} \mathcal{A} \rightarrow \mathcal{H o m}_{\mathcal{O}_{Y}}\left(\pi_{*} \mathcal{A}, \mathcal{O}_{Y}\right)$.

(e) is the case $\mathcal{A}=\mathcal{O}_{X}$ of (d).

COROLlary 1.8.2. (a) U is Frobenius over $\mathfrak{g}^{*(1)}$. In particular, $\mathrm{D}^{b}\left[\bmod ^{c}(\mathrm{U})\right]$ is Calabi-Yau over $\mathfrak{g}^{*(1)}$.

(b) The algebras $\widetilde{D}_{X}$ constructed from torsors in 1.2 .1 are Frobenius over their p-central varieties. In the particular case of the algebras $\widetilde{\mathcal{D}}_{\mathcal{P}}$ associated to partial flag varieties $\mathcal{P}$, the categories $\mathrm{D}^{b}\left[\bmod ^{c}\left(\widetilde{\mathcal{D}}_{\mathcal{P}}\right)\right]$ are CalabiYau over $\mathfrak{g}^{*(1)}$.

(c) $\mathrm{U}^{\lambda}$ is Frobenius over $\mathfrak{g}^{*(1)} \times_{\mathfrak{h}^{*(1)} / W} \operatorname{AS}(\lambda)$ when $\lambda \in \mathfrak{h}^{*}$ is unramified for AS $: \mathfrak{h}^{*} / W \rightarrow \mathfrak{h}^{*(1)} / W$. Also, $\mathcal{D}_{\mathcal{P}}^{\lambda}$ is Frobenius over its $p$-center when $\lambda$ is unramified for $\mathfrak{h}^{*} / W_{\mathcal{P}} \rightarrow \mathfrak{h}^{*(1)} / W_{\mathcal{P}}$. In both cases the derived categories of coherent modules are Calabi-Yau over $\mathfrak{g}^{*(1)} \times_{\mathfrak{h}^{*(1)} / W} \operatorname{AS}(\lambda)$.

Proof. (a) The "regular part" $\mathcal{Z}_{r}=\mathfrak{g}_{\text {reg }}^{*}{ }^{(1)} \times_{\mathfrak{h}^{*(1)} / W} \mathfrak{h}^{*} / W$ of the central variety $\mathcal{Z}(\mathrm{U})$ is smooth since $\mathfrak{h}^{*} / W$ is smooth by [De] and the map $\mathfrak{g}^{*}$ reg $\rightarrow$ $\mathfrak{h}_{r}^{*(1)} / W$ is also smooth [BG, Corollary 3.4]. Since in $\mathcal{Z}(\mathrm{U})$ the Azumaya locus coincides with the smooth locus [BG, Theorems 2.5, 2.6], [BrGo], we see that the algebra $\mathrm{U}$ is Azumaya over $\mathcal{Z}_{r}$, and therefore also Frobenius. The map AS $/ W: \mathfrak{h}^{*} / W \rightarrow \mathfrak{h}^{*(1)} / W$ is canonically Frobenius by part (e) of Lemma 1.8.1, and then $U$ is also Frobenius over $\mathfrak{g}_{\text {reg }}^{*}{ }^{(1)}$ by the part (d) of the same lemma. The complement of $\mathfrak{g}_{\text {reg }}^{*} \subseteq \mathfrak{g}^{*}$ has codimension at least three [BG, Proposition 3.2], so U is Frobenius over all of $\mathfrak{g}^{*(1)}$. Then $\mathrm{D}^{b}\left[\bmod ^{f g}(\mathrm{U})\right]$ is Calabi-Yau over $\mathfrak{g}^{*(1)}$ by the Lemma 1.8.1.b.

(b) The algebras $\widetilde{D}_{X}$ are (locally in $X$ ), tensor products of differential operators and enveloping algebras, so Frobenius structures come from the canonical Frobenius structures for differential operators from Lemma 1.8.1.c, and enveloping algebras from the claim (c). All of the $p$-central varieties involved here are smooth and Calabi-Yau. The algebras $\widetilde{\mathcal{D}}_{\mathcal{P}}$ are in this class and have the additional property that their $p$-central variety $\mathfrak{g}_{\mathcal{P}}^{*(1)}$ are proper over $\mathfrak{g}^{*(1)}$, so we can use Lemma 1.8.1.b.

(c) We only check the Frobenius claim for $\mathrm{U}^{\lambda}$, the rest then follows as in the proof of (b). Since $U$ is Frobenius over $\mathfrak{g}^{*(1)}$ by the part (c), the restriction

$$
\begin{aligned}
& \mathrm{U} \otimes_{\mathcal{O}\left(\mathfrak{g}^{*(1)}\right)}\left[\mathcal{O}\left(\mathfrak{g}^{*(1)}\right) \otimes_{\mathcal{O}\left(\mathfrak{h}^{*(1)} / W\right)} \mathbb{k}_{\mathrm{AS}(\lambda)}\right] \\
& \quad=\mathrm{U} \otimes_{\mathcal{O}\left(\mathfrak{h}^{*} / W\right)}\left[\mathcal{O}\left(\mathfrak{h}^{*} / W\right) \otimes_{\mathcal{O}\left(\mathfrak{h}^{*(1)} / W\right)} \mathbb{k}_{\mathrm{AS}(\lambda)}\right]
\end{aligned}
$$


is Frobenius over $\mathfrak{g}^{*(1)} \times_{\mathfrak{h}^{*(1)} / W} \mathrm{AS}(\lambda)$. This restriction carries the action of the algebra of functions on the fiber of $\mathfrak{h}^{*} / W \rightarrow \mathfrak{h}^{*(1)} / W$ at $\operatorname{AS}(\lambda)$. So each connected component of the fiber of $\mathfrak{h}^{*} / W \rightarrow \mathfrak{h}^{*(1)} / W$ at $\operatorname{AS}(\lambda)$ gives a summand of the restriction, and these summands are again Frobenius. When the map is unramified at $\lambda$ then $\mathrm{U}^{\lambda}$ is one of these summands.

\subsection{Proof of Theorem 1.5.1}

The theorem has already been reduced to the part (a) (see 1.5.3). Since $\mathrm{D}^{b}\left[\bmod ^{c}\left(\widetilde{\mathcal{D}}_{\mathcal{P}}\right)\right]$ is Calabi-Yau with respect to $\mathcal{O}\left(\mathfrak{g}^{*(1)}\right)$ (Corollary 1.8.2), its full triangulated subcategory $\mathrm{D}^{b}\left[\bmod _{\lambda}^{c}\left(\widetilde{\mathcal{D}}_{\mathcal{P}}\right)\right]$ is also Calabi-Yau with respect to $\mathcal{O}\left(\mathfrak{g}^{*(1)}\right)$. Therefore, the equivalence (4) follows from Proposition 1.6.4.b and Lemmas 1.7.2, 1.7.3.

To deduce (5) from (4) we use Lemma 1.6.2(d). It says that the functors $i, \iota$ send the adjunction arrows into adjunction arrows; since $i, \iota$ kill no objects, and the adjunction arrows in $\mathrm{D}^{b}\left[\bmod _{\lambda}^{c}(\widetilde{\mathcal{D}})\right], \mathrm{D}^{b}\left[\bmod _{\lambda}^{f g}(\mathrm{U})\right]$ are isomorphisms, we conclude that the adjunction arrows in $\mathrm{D}^{b}\left[\bmod ^{c}\left(\mathcal{D}^{\lambda}\right)\right]$, $\mathrm{D}^{b}\left[\bmod { }^{f g}\left(\mathrm{U}^{\lambda}\right)\right]$ are isomorphisms, which implies (5).

\subsection{Twisted $\mathcal{D}$-modules on parabolic flags}

Fix a parabolic $P=L J$ with a partial flag variety $\mathcal{P}$. In this subsection we switch attention from $\mathcal{D}_{\mathcal{P}}^{\lambda}, \lambda \in \mathfrak{h}^{*}$, to the more traditional "smaller" sheaf of rings, the sheaf of twisted differential operators on $\mathcal{P}$. Possible twists are indexed by $\lambda \in \operatorname{Pic}(\mathcal{P}) \otimes \mathbb{k}$; recall that $\operatorname{Pic}(\mathcal{P})$ can be identified with the sublattice $\Lambda_{\mathcal{P}} \subset \Lambda$ consisting of the elements $\lambda$ such that $\langle\lambda, \alpha\rangle=0$ if $\alpha$ is a coroot of the Levi ${ }^{(13)}$. For $\lambda \in \Lambda_{\mathcal{P}} \otimes \mathbb{k}$ the corresponding sheaf of rings will be denoted $\mathfrak{D}_{\mathcal{P}}^{\lambda}$. Thus $\mathfrak{D}_{\mathcal{P}}^{\lambda}$ is a central reduction of the sheaf of rings $\widetilde{\mathcal{D}}_{\mathcal{P}}$ associated with the $P /[P, P]$ torsor $G /[P, P]$ over $\mathcal{P}$ as in 1.2.1. The sheaf $\mathfrak{D}_{\mathcal{P}}^{\lambda}$ is related to the sheaf $\mathcal{D}_{\mathcal{P}}^{\lambda}$ considered in the other sections via: $\mathfrak{D}_{\mathcal{P}}^{\lambda}=\mathcal{D}_{\mathcal{P}}^{\lambda} \otimes_{\mathrm{U}^{\lambda}(\overline{\mathfrak{p}})} \mathbb{k}$. For example, for $\mathcal{P}=\mathcal{B}$ we have $\mathfrak{D}_{\mathcal{B}}^{\lambda}=\mathcal{D}_{\mathcal{B}}^{\lambda}$, while for $\mathcal{P}=$ pt, $\Lambda_{\mathcal{P}}=\{0\}$ and we have: $\mathcal{D}_{\mathrm{pt}}^{0}=\mathrm{U}^{0}, \mathfrak{D}_{\mathrm{pt}}^{0}=\mathbb{k}$.

\subsection{1. $\mathfrak{D}_{\mathcal{P}}^{\lambda}$ is derived affine}

The famous result of $[\mathrm{BB} 1]$ asserts that the flag variety $\mathcal{B}$ over a characteristic zero field is $\mathcal{D}_{\mathcal{B}}$-affine, and also $\mathcal{D}_{\mathcal{B}}^{\lambda}$-affine for any semi-ample line bundle $\mathcal{O}_{\lambda}$. This implies that the same is true for the partial flag variety $\mathcal{P}$ : the global sections of a $\mathfrak{D}_{\mathcal{P}}^{\lambda}$-module coincide with the global sections of its

\footnotetext{
${ }^{13}$ Notice that $\Lambda_{\mathcal{P}}$ contains some non-singular weights, due to the $\rho$ shift in the definition of regularity.
} 
pull-back to $\mathcal{B}$; since the pull-back functor is exact and faithful, it is clear that the functor of global sections on the category of $\mathfrak{D}_{\mathcal{P}}^{\lambda}$-modules is exact and faithful, provided that this is known for $\mathcal{D}_{\mathcal{B}}^{\lambda}$ modules. However, for a sheaf of rings $\mathcal{R}$ over an algebraic variety $X$ exactness and faithfulness of the global sections functor for $\mathcal{R}$-modules is equivalent to $X$ being $\mathcal{R}$-affine.

In this subsection we show that a similar fact holds on the level of derived categories in positive characteristic. The material of this section is not used elsewhere in the paper. For simplicity we treat the case of an integral regular weight only, leaving the general case as an exercise to the interested reader. Fix $\lambda \in \Lambda_{\mathcal{P}}$, we will use the same notation for $\lambda \otimes 1 \in \Lambda_{\mathcal{P}} \otimes \mathbb{k}$.

We set $\mathrm{U}_{\mathcal{P}}^{\lambda}=\Gamma\left(\mathfrak{D}_{\mathcal{P}}^{\lambda}\right)$, and let $\pi$ denote the projection $\mathcal{B} \rightarrow \mathcal{P}$. We assume that $p>h$ till the end of the section.

Proposition. Let $\lambda \in \Lambda_{\mathcal{P}} \otimes \mathbb{F}_{p}$ be regular, and assume that $\mathrm{R}^{i} \Gamma\left(\mathfrak{D}_{\mathcal{P}}^{\lambda}\right)=$ 0 for $i>0$. Then $\mathfrak{D}_{\mathcal{P}}^{\lambda}$ is derived affine, i.e. we have an equivalence $\mathrm{R} \Gamma: \mathrm{D}^{b}\left[\bmod ^{c}(\mathfrak{D} \lambda)\right] \stackrel{\cong}{\Longrightarrow} \mathrm{D}^{b}\left[\bmod ^{f g}\left(\mathrm{U}_{\mathcal{P}}^{\lambda}\right)\right]$.

Sketch of proof. To show that a coherent sheaf $\mathcal{R}$ of $\mathcal{O}_{X}$-algebras on a Noetherian scheme $X$ is "unbounded derived affine", i.e., that the derived global sections functor $\mathrm{R} \Gamma$ induces an equivalence between $\mathrm{D}^{-}[\bmod (\mathcal{R})]$ and $\mathrm{D}^{-}[\bmod (\Gamma(\mathcal{R}))]$ it suffices to check that the composition of the global sections functor and the left adjoint localization functor is isomorphic to identity, and that the global sections functor $\mathrm{R} \Gamma$ does not kill any complex of $\mathcal{R}$-modules. To prove a similar statement for the bounded derived category it is enough to verify also that $\mathrm{R} \Gamma(\mathcal{F}) \in \mathrm{D}^{b}[$ Vect $] \Longleftrightarrow \mathcal{F} \in \mathrm{D}^{b}[\bmod (\mathcal{R})]$ for $\mathcal{F} \in \mathrm{D}^{-}[\bmod (\mathcal{R})]$.

Vanishing of $\mathrm{R}^{i} \Gamma\left(\mathfrak{D}_{\mathcal{P}}^{\lambda}\right)$ for $i>0$ implies that $\mathcal{R}=\mathfrak{D}_{\mathcal{P}}^{\lambda}$ satisfies the first of the above conditions. The remaining ones follow directly from the established derived affinity of $\mathcal{D}_{\mathcal{B}}^{\lambda}$, together with the fact that the functor $\pi^{*}: \bmod ^{c}\left(\mathfrak{D}_{\mathcal{P}}^{\lambda}\right) \rightarrow \bmod ^{c}\left(\mathcal{D}_{\mathcal{B}}^{\lambda}\right)$ is exact and commutes with derived global sections. The last claim follows by the projection formula from $\mathrm{R} \pi_{*}\left(\mathcal{O}_{\mathcal{B}}\right)=$ $\mathcal{O}_{\mathcal{P}}$.

Remark 1.10.2. The higher cohomology vanishing for $\mathfrak{D}_{\mathcal{P}}^{\lambda}$ follows from the similar vanishing for $\mathcal{O}_{T^{*} \mathcal{P}}$ by a standard argument [BMR, 3.4.1]. The latter vanishing holds in characteristic zero by the Grauert-Riemenschneider vanishing theorem $[\mathrm{Bro}]$. It is expected to hold in characteristic $p[\mathrm{BrKu}$, 
5.C]. For a fixed dominant $\lambda$ and large $p$ the higher cohomology vanishing for $\mathfrak{D}_{\mathcal{P}}^{\lambda}$ follows from Lemma 1.10 .9 below.

1.10.3. Equivalence class of the Azumaya algebra $\mathfrak{D}_{\mathcal{P}}^{\lambda}$

Set $X=T^{*} \mathcal{P}^{(1)} \times_{\mathcal{P}^{(1)}} \mathcal{B}^{(1)}$, and consider the maps $T^{*} \mathcal{P}^{(1)} \stackrel{\mathrm{pr}}{\longleftarrow} X \stackrel{\iota}{\hookrightarrow}$ $T^{*} \mathcal{B}^{(1)}$. We also have the moment maps $\mu_{\mathcal{P}}: T^{*} \mathcal{P}^{(1)} \rightarrow \mathcal{N}^{(1)}, \mu_{\mathcal{B}}: T^{*} \mathcal{B}^{(1)} \rightarrow$ $\mathcal{N}^{(1)}$.

Recall that the restriction $\mathrm{U}^{-\rho}$ of $\mathrm{U}$ under the embedding $\mathcal{N}^{(1)} \hookrightarrow$ $\mathcal{Z}(\mathrm{U})=\mathfrak{g}^{*(1)} \times_{\mathfrak{h}^{*(1)} / W} \mathfrak{h}^{*} / W, \chi \mapsto(\chi,-\rho)$ is an Azumaya algebra. An isomorphism $\mathcal{D}_{\mathcal{B}}^{-\rho}=\mathcal{D}_{\mathcal{B}}^{(p-1) \rho} \cong \mu_{\mathcal{B}}^{*} \mathrm{U}^{-\rho}$ of Azumaya algebras on $T^{*} \mathcal{B}^{(1)}$ has been explained in [BMR, 5.2].

The sheaf $\mathfrak{D}_{\mathcal{B} \rightarrow \mathcal{P}} \stackrel{\text { def }}{=} \pi^{*}\left(\mathfrak{D}_{\mathcal{P}}\right)$ is a left module for $\mathcal{D}_{\mathcal{B}}$ and a right module for $\pi^{-1} \mathfrak{D}_{\mathcal{P}}$. It is not hard to check that as a module over the center $\mathcal{Z}\left(\mathcal{D}_{\mathcal{B}}\right)=$ $\mathcal{O}_{T^{*} \mathcal{B}^{(1)}}$ the sheaf $\mathcal{D}_{\mathcal{B} \rightarrow \mathcal{P}}$ is supported on the image of $\iota$. Moreover, the action of the center $\pi^{-1} \mathcal{O}_{T^{*} \mathcal{P}^{(1)}} \subset \pi^{-1} \mathcal{D}_{\mathcal{P}}$ is compatible with the action of $\mathcal{O}_{T^{*} \mathcal{B}^{(1)}}$ via pr, thus $\mathcal{D}_{\mathcal{B} \rightarrow \mathcal{P}}$ is a module over $\iota^{*}\left(\mathcal{D}_{\mathcal{B}}\right) \otimes_{\mathcal{O}_{X}} \operatorname{pr}^{*}\left(\mathfrak{D}_{\mathcal{P}}^{\text {op }}\right)$. According to [BrBr, Proposition 3.7] or [OV, Theorem 2.4], this bimodule provides an equivalence between the two Azumaya algebras $\iota^{*}\left(\mathcal{D}_{\mathcal{B}}\right)$ and $\operatorname{pr}^{*}\left(\mathfrak{D}_{\mathcal{P}}\right)$. Hence, for line bundles $\mathcal{O}_{\mathcal{B}, \nu}$ on $\mathcal{B}$ and $\mathcal{O}_{\mathcal{P}, \lambda}$ on $\mathcal{P}$ the sheaf

$$
\nu \mathfrak{D}_{\mathcal{B} \rightarrow \mathcal{P}}^{\lambda} \stackrel{\text { def }}{=} \mathcal{O}_{\mathcal{B}, \nu} \otimes_{\mathcal{O}_{\mathcal{B}}} \mathfrak{D}_{\mathcal{B} \rightarrow \mathcal{P}} \otimes_{\pi^{-1} \mathcal{O}_{\mathcal{P}}} \pi^{-1} \mathcal{O}_{\mathcal{P},-\lambda}
$$

is a left module for $\iota^{*} \mathcal{D}_{\mathcal{B}}^{\nu}$ and a right module for $\mathrm{pr}^{*} \mathfrak{D}_{\mathcal{P}}^{\lambda}$, providing an equivalence between the two Azumaya algebras. In particular, for $\nu=(p-1) \rho$ we get an equivalence between $\mathcal{D}_{\mathcal{B}}^{(p-1) \rho} \cong \mu_{\mathcal{B}}^{*}\left(\mathrm{U}^{-\rho}\right)$ and $\operatorname{pr}^{*}\left(\mathfrak{D}_{\mathcal{P}}^{\lambda}\right)$.

LEmma. For every $\lambda \in \Lambda_{\mathcal{P}}$ there exists a unique (up to a unique isomorphism) module $\mathcal{M}$ for $\mu_{\mathcal{P}}^{*}\left(\mathrm{U}^{-\rho}\right) \otimes_{\mathcal{O}_{T^{*} \mathcal{P}}(1)}\left(\mathfrak{D}_{\mathcal{P}}^{\lambda}\right)^{\text {op }}$ together with an isomorphism of bimodules $\operatorname{pr}^{*}(\mathcal{M}) \cong(p-1) \rho \mathfrak{D}_{\mathcal{B} \rightarrow \mathcal{P}}^{\lambda}$, where we have used the identification $\mathrm{pr}^{*} \mu_{\mathcal{P}}^{*} \mathrm{U}^{-\rho}=\mu_{\mathcal{B}}^{*} \mathrm{U}^{-\rho} \cong \mathcal{D}_{\mathcal{B}}^{(p-1) \rho}$. This module provides an equivalence between Azumaya algebras $\mu_{\mathcal{P}}^{*}\left(\mathrm{U}^{-\rho}\right)$ and $\mathfrak{D}_{\mathcal{P}}^{\lambda}$.

Proof. First we show that there is some equivalence between $\mu_{\mathcal{P}}^{*}\left(\mathrm{U}^{-\rho}\right)$ and $\mathfrak{D}_{\mathcal{P}}^{\lambda}$. It follows from [Mln, IV.2, Corollary 2.6] that such an equivalence exists, provided that it exists on an open dense subvariety of $T^{*} \mathcal{P}^{(1)}$. However, over an open dense subvariety the map pr admits a section, thus the sought for equivalence comes from the bimodule $(p-1) \rho \mathfrak{D}_{\mathcal{B} \rightarrow \mathcal{P}}^{\lambda}$. 
Let now $\mathcal{M}^{\prime}$ be some bimodule providing an equivalence between $\mu_{\mathcal{P}}^{*}\left(\mathrm{U}^{-\rho}\right)$ and $\mathfrak{D}_{\mathcal{P}}^{\lambda}$. Then $\operatorname{pr}^{*} \mathcal{M}^{\prime} \cong \mathcal{L} \otimes_{\mathcal{O}_{X}}{ }^{(p-1) \rho} \mathfrak{D}_{\mathcal{B} \rightarrow \mathcal{P}}^{\lambda}$ for some line bundle $\mathcal{L}$ on $X$, because a splitting bundle for an Azumaya algebra is defined uniquely up to twist by a line bundle. Existence of $\mathcal{M}$ would follow once we show that $\mathcal{L} \cong \operatorname{pr}^{*} \mathcal{L}^{\prime}$ for some line bundle $\mathcal{L}^{\prime}$ on $T^{*} \mathcal{P}^{(1)}$. Since the Picard group of the total space of a vector bundle is isomorphic to the Picard group of the base, it is sufficient to see that the restriction of $\mathcal{L}$ to the zero section $\mathcal{B}^{(1)} \subset X$ is a pull-back under $\pi^{(1)}$. Furthermore, since the quotient $\Lambda / \Lambda_{\mathcal{P}}$ is torsion free, it is enough to check that the vector bundle $\mathcal{O}_{\mathcal{B}^{(1)}} \otimes_{\mathcal{O}_{X}}{ }^{(p-1) \rho} \mathfrak{D}_{\mathcal{B} \rightarrow \mathcal{P}}^{\lambda}$ on $\mathcal{B}^{(1)}$ is a pull-back under $\pi^{(1)}$. The latter bundle is readily identified with $\mathcal{H o m}\left(\left(\pi^{(1)}\right)^{*} \operatorname{Fr}_{*}\left(\mathcal{O}_{\mathcal{P}, \lambda}\right), \operatorname{Fr}_{*}\left(\mathcal{O}_{\mathcal{B},(p-1) \rho}\right)\right)$, cf. [BMR, 2.2.5]. Since the vector bundle $\operatorname{Fr}_{*}\left(\mathcal{O}_{\mathcal{B},(p-1) \rho}\right)$ on $\mathcal{B}^{(1)}$ is trivial, we have proved the existence of $\mathcal{M}$. Its uniqueness up to a unique isomorphism follows from the fact that $\mathrm{pr}^{*}$ is fully faithful on the abelian category of quasi-coherent sheaves, which is clear from $\pi_{*} \mathcal{O}_{\mathcal{B}}=\mathcal{O}_{\mathcal{P}}$.

Corollary 1.10.4. Let $\lambda \in \Lambda_{\mathcal{P}}$ satisfy the assumptions of Proposition 1.10.1, and $\chi \in \mathcal{N}^{(1)}$ be in the image of $\mu_{\mathcal{P}}$. Then we have a canonical equivalence of triangulated categories

$$
\mathrm{D}^{b}\left[\bmod _{\chi}^{f g}\left(\mathrm{U}_{\mathcal{P}}^{\lambda}\right)\right] \cong \mathrm{D}^{b}\left[\mathcal{C o h}_{\chi}\left(T^{*} \mathcal{P}^{(1)}\right)\right],
$$

where the subindex $\chi$ denotes, respectively, the full subcategory of modules with the action of the central subalgebra $\Gamma\left(\mathcal{O}_{\left.T^{*} \mathcal{P}^{(1)}\right)}\right)$ having generalized central character defined by $\chi$ and the full subcategory of sheaves settheoretically supported on $\mu_{\mathcal{P}}^{-1}(\chi)$.

We denote the inverse of this equivalence by $\mathcal{F} \mapsto M_{\mathcal{F}}^{\lambda}$.

Remark 1.10.5. It follows from the construction that for $\chi=0$ the splitting bundle for $\mathfrak{D}_{\mathcal{P}}^{\lambda}$ on the formal neighborhood of $\mu_{\mathcal{P}}^{-1}(0)$, that we used to construct the equivalence, restricts on the zero section $\mathcal{P}^{(1)} \subset T^{*} \mathcal{P}^{(1)}$ to the bundle $\operatorname{Fr}_{*}\left(\mathcal{O}_{\mathcal{P}, \lambda}\right)$.

It is explained in Remark 1.3.5 above that for $\mathcal{P}=\mathcal{B}$ an equivalence as in the last corollary can be fixed by fixing a weight $\nu$ such that $\nu+\lambda$ is unramified. Comparing the definitions we see that the equivalence of corollary corresponds to the choice $\nu=(p-1) \rho-\lambda$.

EXAMPLE 1.10.6. Let $G=S L(n+1), \mathcal{P}=\mathbb{P}^{n}$. It is easy to see that $\mathrm{R}^{i} \Gamma\left(\mathcal{O}_{T^{*} \mathbb{P}^{n}}\right)=0$ for $i>0$, thus Proposition 1.10.1 applies for line bundles corresponding to weights, which are regular modulo $p$. 
A line bundle $\mathcal{O}(i)$ on $\mathbb{P}^{n}$ corresponds to the weight $i \omega$, where $\omega$ is a fundamental weight of $S L(n+1)$. The weight $i \omega$ modulo $p$ is regular if and only if $i \neq j \bmod p$ for $-n \leq j \leq-1$. Without loss of generality assume that $0 \leq i<p-n$.

Fix $\chi=0$. For $\mathcal{F} \in \mathrm{D}^{b}\left[\mathcal{C o h}_{\mu_{\mathcal{P}}^{-1}(0)}\left(T^{*} \mathbb{P}^{n(1)}\right)\right]$ we have $M_{\mathcal{F}}=R \Gamma(\mathcal{E} \otimes \mathcal{F})$, where $\mathcal{E}$ is the splitting bundle for $\mathcal{D}_{\mathbb{P}^{n}}^{i \omega}$ on the formal neighborhood of the zero section in $T^{*} \mathbb{P}^{n(1)}$. According to the previous remark, $\left.\mathcal{E}\right|_{\mathbb{P} n(1)} \cong$ $\operatorname{Fr}_{*}\left(\mathcal{O}_{\mathbb{P}^{n}}(i)\right)$. By [HKR, Proposition 4.1], $\operatorname{Fr}_{*}(\mathcal{O}(i)) \cong \bigoplus_{j=0}^{n} \mathcal{O}_{\mathbb{P}^{n}}(-j)^{\oplus d_{j}}$ for some $d_{j}>0$. We claim that $\mathcal{E} \cong \bigoplus_{j=0}^{n} \widehat{\mathcal{O}}(-j)^{\oplus d_{j}}$, where $\widehat{\mathcal{O}}$ stands for the structure sheaf of the formal neighborhood of the zero section in $T^{*} \mathbb{P}^{n(1)}$. To show this one can use induction in $m$ to construct an isomorphism on the $m$-th neighborhood of the zero section. The obstruction on the $m$-th step lies in $\mathrm{H}^{1}\left(\mathbb{P}^{n}, \operatorname{Sym}^{m}\left(\mathcal{T}_{\mathbb{P} n}\right) \otimes \mathcal{E} \operatorname{nd}\left(\operatorname{Fr}_{*}(\mathcal{O}(i))\right)\right)$. It is not hard to show that $\mathrm{H}^{>0}\left(\mathcal{O}_{T^{*} \mathbb{P}^{n}}(j)\right)=0$ for $-n \leq j \leq n$, thus the obstruction vanishes.

It follows that the coherent sheaves corresponding to indecomposable projective pro-objects in $\bmod _{0}\left(\mathrm{U}_{\mathbb{P} n}^{i \omega}\right)$ are the line bundles $P_{j}=\widehat{\mathcal{O}}(j), j=$ $0, \ldots, n$. The objects $L_{k}$ in the derived category of coherent sheaves corresponding to irreducible $\mathrm{U}_{\mathbb{P}^{n}}^{i \omega}$ modules are characterized by $\operatorname{RHom}\left(P_{j}, L_{k}\right)=$ $\mathbb{k}^{\delta_{j k}}$. Thus we have $L_{j}=\mathfrak{i}_{*} \Omega_{\mathbb{P}^{n}}^{j}(j)[j]$, where $\mathfrak{i}$ stands for the embedding of the zero section.

Remark. The same objects $\Omega_{\mathbb{P}^{n}}^{j}(j)[j]$ correspond to irreducible objects in $\mathcal{C o h}^{\mathbb{Z}}{ }^{n+1}\left(\mathbb{A}^{n+1}\right)$ under an equivalence between $\mathrm{D}^{b}\left[\mathcal{C o h}^{\mathbb{Z}_{n+1}}\left(\mathbb{A}^{n+1}\right)\right]$ and the derived category of coherent sheaves on the total space of canonical bundle on $\mathbb{P}^{n}$, cf., e.g., [R, 4.3].

\subsubsection{Compatibility}

For $\lambda \in \Lambda_{\mathcal{P}}$ we have the following exact functors between abelian categories:

$$
\mathcal{C o h}_{\mu_{\mathcal{P}}^{-1}(\chi)}\left(T^{*} \mathcal{P}^{(1)}\right) \cong \bmod _{\chi}\left(\mathfrak{D}_{\mathcal{P}}^{\lambda}\right) \stackrel{\pi^{*}}{\longrightarrow} \bmod _{\chi}\left(\mathcal{D}_{\mathcal{B}}^{\lambda}\right) \cong \mathcal{C o h}_{\mathcal{B}_{\chi}}\left(T^{*} \mathcal{B}^{(1)}\right)
$$

where the equivalences come from the canonical splitting arising from Lemma 1.10.3. The definition of equivalences implies that the composition functor is identified with $\iota_{*} \operatorname{pr}^{*}: \mathcal{C o h}_{\mu_{\mathcal{P}}^{-1}(\chi)}\left(T^{*} \mathcal{P}^{(1)}\right) \rightarrow \mathcal{C o h}_{\mathcal{B}_{\chi}}\left(T^{*} \mathcal{B}^{(1)}\right)$.

Since $\mathrm{R} \Gamma \circ \pi^{*}(\mathcal{M}) \cong \mathrm{R} \Gamma(\mathcal{M})$ for $\mathcal{M} \in \mathcal{D}_{\mathcal{P}}^{\lambda}$ we arrive at the following

Proposition. Fix $\mathcal{P}, \lambda \in \Lambda_{\mathcal{P}}$ satisfying the assumptions of Proposition 1.10.1. Let $\phi_{\mathcal{P}}^{\lambda}: \mathrm{U}^{\lambda} \rightarrow \mathrm{U}_{\mathcal{P}}^{\lambda}$ be the map coming from the action of $\mathfrak{g}$ on 
$\mathcal{P}$. Then for $\mathcal{F} \in \mathrm{D}^{b}\left[\mathcal{C o h}_{\mu_{\mathcal{P}}^{-1}(\chi)}\left(T^{*} \mathcal{P}^{(1)}\right)\right]$ we have a canonical isomorphism $\left(\phi_{\mathcal{P}}^{\lambda}\right)^{*}\left(M_{\mathcal{F}}^{\lambda}\right) \cong M_{\iota *}^{\lambda} \mathrm{pr}^{*} \mathcal{F}$.

Remark 1.10.8. Assume that the map $\phi_{\mathcal{P}}^{\lambda}$ is surjective. Then for an irreducible $\mathrm{U}_{\mathcal{P}}^{\lambda}$-module $M$, the module $\left(\phi_{\mathcal{P}}^{\lambda}\right)^{*}(M)$ is also irreducible. Thus in this case the previous proposition can be used to describe explicitly the image of an irreducible $\mathrm{U}^{\lambda}$-module under the equivalence of $[\mathrm{BMR}]$, once the image of an irreducible $\mathrm{U}_{\mathcal{P}}^{\lambda}$ module in $\mathrm{D}^{b}\left[\mathcal{C} \mathrm{oh}\left(T^{*} \mathcal{P}^{(1)}\right)\right]$ is known. For example, if $G=S L(n+1), \mathcal{P}=\mathbb{P}^{n}$, then Lemma 1.10.9.a below implies surjectivity, thus Example 1.10.6 yields an explicit description of $n+1$ out of the total $(n+1)$ ! irreducible objects.

We finish the section by giving a sufficient criterion for surjectivity of $\phi_{\mathcal{P}}^{\lambda}$

Lemma 1.10.9. a) Suppose that the map $\mu_{\mathcal{P}}$ is birational onto its image, and the image is a normal variety. Then the map $\phi_{\mathcal{P}}^{\lambda}$ is surjective for any $\lambda \in \Lambda_{\mathcal{P}}$.

b) Fix $\lambda \in \Lambda_{\mathcal{P}}$, such that $\lambda+\rho$ is dominant. There exists (an explicitly computable) $N \in \mathbb{Z}$ depending on the type of $G$ and on $\lambda$, such that $\mathrm{R}^{i} \Gamma\left(\mathfrak{D}_{\mathcal{P}}^{\lambda}\right)=0$ for $i>0$ and $\phi_{\mathcal{P}}^{\lambda}$ is surjective, provided that $p=\operatorname{char}(\mathbb{k})>N$.

Sketch of proof. The assumptions in (a) imply that the map $\mu_{\mathcal{P}}^{*}$ : $\operatorname{Sym}(\mathfrak{g}) \rightarrow \Gamma\left(\mathcal{O}_{T^{*} \mathcal{P}}\right)$ is surjective. This yields surjectivity of $\phi_{\mathcal{P}}^{\lambda}$ by a wellknown argument, cf., e.g., [BMR, 3.4.1].

To show (b) observe that $\mathrm{R}^{i} \Gamma\left(\mathfrak{D}_{\mathcal{P}}^{\lambda}\right)=\mathrm{R}^{i} \Gamma\left(\pi^{*} \mathfrak{D}_{\mathcal{P}}^{\lambda}\right)$, while the map $\phi_{\mathcal{P}}^{\lambda}$ can be obtained from the canonical surjection of sheaves $\mathcal{D}_{\mathcal{B}}^{\lambda} \rightarrow \pi^{*}\left(\mathfrak{D}_{\mathcal{P}}^{\lambda}\right)$ by applying the functor of global sections. This map of sheaves can be extended to a resolution of $\pi^{*}\left(\mathfrak{D}_{\mathcal{P}}^{\lambda}\right)$ by locally projective $\mathcal{D}_{\mathcal{B}}^{\lambda}$ modules, coming from the relative De Rham complex of the map $\pi$. The terms of this resolution are of the form $\mathcal{D}_{\mathcal{B}}^{\lambda} \otimes_{\mathcal{O}_{\mathcal{B}}} \Lambda^{i}\left(\mathcal{T}_{\mathcal{B} \rightarrow \mathcal{P}}\right)$ placed in degree $-i$, where $\mathcal{T}_{\mathcal{B} \rightarrow \mathcal{P}}$ is the kernel of the differential $d \pi$. Thus desired surjectivity and cohomology vanishing follow from cohomology vanishing $\mathrm{H}^{>0}\left(\mathcal{D}_{\mathcal{B}}^{\lambda} \otimes_{\mathcal{O}_{\mathcal{B}}} \Lambda^{i}\left(\mathcal{T}_{\mathcal{B} \rightarrow \mathcal{P}}\right)\right)=0$ for all $i$, which can be established by the method of [BB1]. Namely, we choose a dominant $\nu \in \Lambda$, such that $\mathrm{H}^{>0}\left(\mathcal{O}_{T^{*} \mathcal{B}} \otimes_{\mathcal{O}_{\mathcal{B}}} \Lambda^{i}\left(\mathcal{T}_{\mathcal{B} \rightarrow \mathcal{P}}\right) \otimes_{\mathcal{O}_{\mathcal{B}}} \mathcal{O}_{\mathcal{B}, \nu}\right)=0$, it suffices that $\nu+\alpha$ is dominant when $\alpha$ is a sum of some subset of positive roots in the Levi $\bar{P}$. Then $\mathrm{H}^{>0}\left[\mathcal{O}_{\mathcal{B}, \nu} \otimes_{\mathcal{O}_{\mathcal{B}}} \mathcal{D}_{\mathcal{B}}^{\lambda} \otimes_{\mathcal{O}_{\mathcal{B}}} \Lambda^{i}\left(\mathcal{T}_{\mathcal{B} \rightarrow \mathcal{P}}\right)\right]=0$. Thus we will be done if we show that for a $\mathcal{D}_{\mathcal{B}}^{\lambda}$-module $\mathcal{M}$, the sheaf $\mathcal{M}$ is a direct summand in the sheaf $V \otimes_{\mathbb{k}} \mathcal{O}_{\mathcal{B}, \nu} \otimes_{\mathcal{O}_{\mathcal{B}}} \mathcal{M}$, where $V$ is the irreducible 
representation of $G$ with lowest weight $-\nu$. We have an action of $\mathfrak{g}$ on this sheaf, preserving the filtration arising from the canonical filtration of $V \otimes \mathcal{O}_{\mathcal{B}}$ by line bundles. The associated graded sheaves of the filtration are of the form $\mathcal{O}_{\mathcal{B}, \eta+\nu} \otimes_{\mathcal{O}_{\mathcal{B}}} \mathcal{M}, \eta \in \mathrm{wt}(V)$, where $\mathrm{wt}(V)$ is the set of weights of $V$. The Harish-Chandra center acts on such a subquotient via the character corresponding to the $W_{\text {aff }}^{\prime}$ orbit of $\eta+\nu+\lambda$. Thus if $\lambda$ is the only element in $(\lambda+\nu+\operatorname{wt}(V)) \cap W_{\text {aff }}^{\prime} \bullet \lambda$, then $\mathcal{M}$ is indeed a direct summand in $V \otimes_{\mathbb{k}} \mathcal{O}_{\mathcal{B}, \nu} \otimes_{\mathcal{O}_{\mathcal{B}}} \mathcal{M}$. Since $\lambda+\rho$ is dominant, we have $w \bullet(\lambda) \preceq \lambda$ for any $w \in W$, while $\eta \succeq-\nu$ for any $\eta \in \operatorname{wt}(V)$. Thus $(\lambda+\nu+\operatorname{wt}(V)) \cap W \bullet \lambda=\{\lambda\}$. There is only a finite number of primes dividing one of the finite number of nonzero weights $\eta+\nu+\lambda-w \bullet \lambda, w \in W, \eta \in \operatorname{wt}(V), \eta \neq-\nu$. For $p$ outside of this finite set we have $\{\lambda\}=(\lambda+\nu+\operatorname{wt}(V)) \cap W_{\text {aff }}^{\prime} \bullet \lambda$.

\section{§2. Affine braid group action on $\mathfrak{g}$-modules by intertwining func- tors}

Here we develop a characteristic $p$ version of the theory of intertwining functors of Beilinson and Bernstein [BB2], see also [BeG], [Mi] for results in characteristic zero which are parallel to many of the results in this section.

For each regular integral weight $\lambda$ we construct a canonical action of an incarnation ${ }^{\Theta} B^{\prime}$ aff of the (extended) affine braid group $B_{\text {aff }}^{\prime}$ on the category $\mathrm{D}^{b}\left[\bmod _{\lambda}^{f g}(\mathrm{U})\right]$. The group ${ }^{\Theta} B_{\text {aff }}^{\prime}$ depends on a regular orbit $\Theta=W_{\text {aff }}^{\prime} \lambda$; it is non canonically isomorphic to $B_{\text {aff }}^{\prime}$. The action is generated by intertwining functors; they intertwine different localization functors.

The localization equivalences allow us to translate the action of ${ }^{\Theta} B_{\text {aff }}^{\prime}$ from $\mathrm{D}^{b}\left[\bmod _{\lambda}^{f g}(\mathrm{U})\right]$ to $\mathrm{D}^{b}\left[\bmod _{\lambda}^{c}(\widetilde{\mathcal{D}})\right]$. Here the group that naturally acts on $\mathrm{D}^{b}\left[\bmod _{\lambda}^{c}(\widetilde{\mathcal{D}})\right]$ is always $B_{\text {aff }}^{\prime}$. Moreover, the categories $\bmod _{\lambda}^{c}(\widetilde{\mathcal{D}})$ for different weights $\lambda$ are canonically identified by tensoring with line bundles; this identification is compatible with the $B_{\text {aff }}^{\prime}$ action (Corollary 2.1.6).

In the remainder we assume that $p>h$; recall that regular weights exist only for $p \geq h$, the case $p=h$ is excluded to avoid $G=S L(p)$ which violates assumption (C) from 1.1.3. Since exponents of $W$ do not exceed $h$, this also implies that $p$ does not divide $|W|$.

\subsubsection{Further extensions}

In this section we do not work in the maximal possible and perhaps even not in the maximal reasonable generality. For example, translation functors could be defined and probably described geometrically for modules with non-integral Harish-Chandra central characters. This would, however, 
further complicate the notations without yielding new ideas or obvious applications, thus we did not pursue it.

In this paper the action of the braid group is introduced on derived categories of modules with a generalized Harish-Chandra central character. One can, in fact, define it also for the derived categories of modules with a fixed Harish-Chandra central character. This technical variation will be discussed in a future publication.

\subsection{Affine braid group actions: statement}

We start with recalling some standard material on affine Weyl groups.

2.1.1. Affine Weyl and braid groups

The groups $W, W_{\text {aff }}$ and some related objects were introduced in 1.1.2. These groups are Coxeter groups (with the sets of simple reflections $I, I_{\text {aff }}$ respectively), so we can consider the corresponding braid groups $B, B$ aff .

These algebraic constructions have a topological interpretation: $B=$ $\left.\pi_{1}\left(\left(\mathfrak{h}_{\mathbb{C}}^{*}\right)^{\mathrm{reg}}\right) / W\right), B_{\mathrm{aff}}=\pi_{1}\left(\left(\check{H}_{s c}\right)^{\mathrm{reg}} / W\right)$ where $\check{H}_{s c}=\check{H}_{s c}(\mathbb{C})$ is the torus dual to the Cartan subgroup $H_{a d j}$ of the adjoint group $G_{a d j}=G / Z(G)$.

For $\alpha \in I_{\text {aff }}$ let $s_{\alpha} \in W_{\text {aff }}, \widetilde{s}_{\alpha} \in B_{\text {aff }}$ denote the corresponding standard generator.

We have an embedding of sets $C: W_{\text {aff }} \hookrightarrow B_{\text {aff }}$, also denoted by $w \mapsto$ $\widetilde{w}$, where for a minimal length decomposition $w=s_{\alpha_{1}} \cdots s_{\alpha_{\ell(w)}}$ we have $C(w)=\widetilde{w}=\widetilde{s}_{\alpha_{1}} \cdots \widetilde{s}_{\alpha_{\ell(w)}}$. The map $C$ is not a group homomorphism; however, we have $C\left(w_{1} \cdot w_{2}\right)=C\left(w_{1}\right) \cdot C\left(w_{2}\right)$ when the lengths add up, i.e., $\ell\left(w_{1} w_{2}\right)=\ell\left(w_{1}\right)+\ell\left(w_{2}\right)$, where $\ell: W_{\text {aff }} \rightarrow \mathbb{Z}_{\geq 0}$ is the length function sending $w$ to the length of its minimal decomposition. This happens, in particular, when $w_{1}=\lambda_{1}, w_{2}=\lambda_{2}$ lie in the semigroup $Q_{\text {dom }}$ of dominant weights in $Q$ (we have $Q_{\text {dom }} \subset Q \subset W_{\text {aff }}$ ). The image of $C$ generates $B$ aff, and it is known that $B_{\text {aff }}$ can be defined by generators $\widetilde{w}, w \in W_{\text {aff }}$, and relations $\widetilde{u v}=\widetilde{u v}$ when lengths add up:

$$
\widetilde{u v}=\tilde{u} \cdot \tilde{v} \quad \text { when } \ell(u)+\ell(v)=\ell(u v) .
$$

The length function admits a natural extension to the extended affine Weyl group $W_{\text {aff }}^{\prime}$, which is characterized by $\ell(w \omega)=\ell(w)$ if $w \in W_{\text {aff }}$, and $\omega \in \Omega \stackrel{\text { def }}{=} \operatorname{Stab}_{W_{\text {aff }}^{\prime}}\left(A_{0}\right)$. The extended affine braid group $B_{\text {aff }}^{\prime}$ is defined by generators $\tilde{w}, w \in W_{\text {aff }}^{\prime}$, and relations (6). 
Then one finds that $B_{\text {aff }}^{\prime}$ is the semidirect product $B_{\text {aff }} \rtimes \Omega$, where the finite abelian group $\Omega=\operatorname{Stab}_{W_{\text {aff }}^{\prime}}\left(A_{0}\right) \cong \Lambda / Q=W_{\text {aff }}^{\prime} / W_{\text {aff }}$ acts on $B_{\text {aff }}$ via its action on the set of simple reflection $I_{\text {aff }}$.

\subsubsection{Local action and the affine braid group}

For each $\alpha \in I_{\text {aff }}$, each alcove $A$ has a unique face of type $\alpha$. We denote by $*$ the right action of $W_{\text {aff }}$ on the set of alcoves such that for $\alpha \in I_{\text {aff }}$ the alcove $A * s_{\alpha}$ is the reflection $s_{F}(A)$ of $A$ in the unique face $F$ of $A$ of type $\alpha$ (cf., e.g., [Lu1, 1.1]). We also get a free $*$-action on the set of regular characters $\Lambda_{\text {reg }}$, i.e., the ones which lie in alcoves, the quotient is identified with the set of characters in the open fundamental alcove.

We will need to twist these constructions by certain torsors $\Theta$ for the affine Weyl group as follows.

Let $\Theta \subset \Lambda_{\mathbb{R}}=\Lambda \otimes \mathbb{R}$ be a free orbit of $W_{\text {aff }}^{\prime}$. We define the local extended affine Weyl group ${ }^{\Theta} W_{\text {aff }}^{\prime}$ as the group of permutations of the set $\Theta$ which commute with the action of $W_{\text {aff }}^{\prime}$. We endow ${ }^{\ominus} W_{\text {aff }}^{\prime}$ with the composition law opposite to the composition of permutations; thus the action of ${ }^{\Theta} W_{\text {aff }}^{\prime}$ on $\Theta$ is a right action, we denote it by $w: \lambda \mapsto \lambda * w$. Every choice of $\lambda \in \Theta$ defines an isomorphism $\operatorname{isom}_{\lambda}: W_{\text {aff }}^{\prime} \cong{ }^{\Theta} W_{\text {aff }}^{\prime}$. The image of the normal subgroup $W_{\text {aff }} \subset W_{\text {aff }}^{\prime}$ under isom ${ }_{\lambda}$ does not depend on $\lambda \in \Theta$, thus we get a normal subgroup ${ }^{\Theta} W_{\text {aff }}=\operatorname{isom}_{\lambda}\left(W_{\text {aff }}\right) \subset{ }^{\Theta} W_{\text {aff }}^{\prime}$.

We use the isomorphism isom ${ }_{\lambda}$ for $\lambda$ in the fundamental alcove $A_{0}$ to endow ${ }^{\Theta} W_{\text {aff }}$ with a structure of a Coxeter group; an element $s \in{ }^{\Theta} W_{\text {aff }}$ is a simple reflection if and only if it sends every $\lambda \in \Theta$ to an element which is symmetric to $\lambda$ with respect to a face of the alcove containing $\lambda$. The set of simple reflections ${ }^{\Theta} I_{\text {aff }} \subset{ }^{\Theta} W_{\text {aff }}$ is in a canonical bijection with the set of pairs $(\lambda, F)$ where $\lambda \in \Theta$, and $F$ is a face of the alcove of $\lambda$, modulo the action of $W_{\text {aff }}^{\prime}$. A choice of $\lambda \in \Theta$ defines a bijection ${ }^{\Theta} I_{\text {aff }} \cong I_{\text {aff }}$; in fact, this bijection depends only on the $W_{\text {aff }}$ orbit of $\lambda$, and one easily sees that bijections corresponding to $\lambda, \mu \in \Theta$ lying in different $W_{\text {aff }}$ orbits differ by composition with an element of the abelian group $\Omega \cong W_{\text {aff }}^{\prime} / W_{\text {aff acting on }}$ $I_{\text {aff }}$.

One can define the length function $\ell:{ }^{\Theta} W_{\text {aff }}^{\prime} \rightarrow \mathbb{Z}_{\geq 0}$ in such a way that $\ell\left(\operatorname{isom}_{\lambda}(w)\right)=\ell(w)$ for any $\lambda \in A_{0} \cap \Theta$. It can be described in a more intrinsic way as follows: for any $\lambda \in \Theta$ and $w \in{ }^{\Theta} W_{\text {aff }}^{\prime}$ the number of affine coroot hyperplanes $H_{\check{\alpha}, n}$ separating $\lambda$ and $w(\lambda)$ equals $\ell(w)$. We can then define the braid groups ${ }^{\Theta} B_{\text {aff }},{ }^{\ominus} B_{\text {aff }}^{\prime}$ by generators $\widetilde{w}, w \in{ }^{\Theta} W_{\text {aff }}$ and 
relations (6). Thus ${ }^{\Theta} B_{\text {aff }}^{\prime}$ comes with a canonical lifting map ${ }^{\Theta} C:{ }^{\Theta} W_{\text {aff }}^{\prime} \rightarrow$ ${ }^{\Theta} B_{\mathrm{aff}}^{\prime}, w \mapsto \tilde{w}$.

Remark. Notice that, although our description involved the choice of an initial alcove $A_{0}$, the group ${ }^{\Theta} W_{\text {aff }}$ with its Coxeter structure, and the groups ${ }^{\Theta} W_{\text {aff }}^{\prime},{ }^{\Theta} B_{\text {aff }},{ }^{\Theta} B_{\text {aff }}^{\prime}$ can be constructed in a canonical way from the affine Euclidean space $\Lambda \otimes \mathbb{R}$ subdivided into alcoves with a fixed subset $\Theta \subset \Lambda \otimes \mathbb{R}$ (unlike the Coxeter structure on the group $W_{\text {aff }}$, which depends on the choice of an alcove).

2.1.3. We proceed to state the main results of this chapter.

For $w \in{ }^{\Theta} W_{\text {aff }}^{\prime}$ and $\lambda \in \Theta$ we will say that $w$ increases $\lambda$ if $w=$ $s_{1} \cdots s_{n} \omega$ where $\ell(\omega)=0, s_{i} \in{ }^{\Theta} I_{\mathrm{aff}}$, and $\lambda * s_{1} \cdots s_{i}<\lambda * s_{1} \cdots s_{i+1}$ for every $i=1, \ldots, n$.

The set of integral Harish-Chandra central characters is identified with $\mathfrak{h}_{\mathbb{F}_{p}}^{*} / W=\Lambda / W_{\text {aff }}^{\prime}$. We fix an integral regular Harish-Chandra central character and let $\Theta$ be the corresponding free orbit of $W_{\text {aff }}^{\prime}$ in $\Lambda$. We set $\bmod _{\Theta}(\mathrm{U})=\bmod _{\lambda}(\mathrm{U}), \lambda \in \Theta$.

For $\lambda \in \Theta$ we have the category of twisted $D$-modules $\bmod _{\lambda}^{c}\left(\widetilde{\mathcal{D}}_{\mathcal{B}}\right)$, the category $\bmod _{\lambda}^{f g}(\mathrm{U})$, the equivalence $\mathrm{R}_{\widetilde{\mathcal{D}}_{\mathcal{B}}, \lambda}: \mathrm{D}^{b}\left[\bmod _{\lambda}^{c}\left(\widetilde{\mathcal{D}}_{\mathcal{B}}\right)\right] \rightarrow$ $\mathrm{D}^{b}\left[\bmod _{\Theta}^{f g}(\mathrm{U})\right]$ and the inverse equivalence $\mathcal{L}^{\widehat{\lambda}}$, see [BMR, Theorem 3.2].

For $\lambda, \mu \in \Lambda$ we have an equivalence $\bmod _{\lambda}^{c}\left(\widetilde{\mathcal{D}}_{\mathcal{B}}\right) \rightarrow \bmod _{\mu}^{c}\left(\widetilde{\mathcal{D}}_{\mathcal{B}}\right)$ sending $\mathcal{F}$ to $\mathcal{O}_{\mu-\lambda} \otimes_{\mathcal{O}_{\mathcal{B}}} \mathcal{F}$; we will denote it by Tens $\operatorname{Te}_{\mu, \lambda}$.

TheOREM 2.1.4. There is a unique action (14) of ${ }^{\Theta} B_{\text {aff }}^{\prime}$ on $\mathrm{D}^{b}\left[\bmod _{\Theta}(\mathrm{U})\right], b \mapsto \mathbf{I}_{b}$, such that whenever $w \in{ }^{\Theta} W_{\text {aff }}^{\prime}$ increases some $\lambda \in \Theta$ we have

$$
\operatorname{Tens}_{\lambda, \lambda * w} \circ \mathcal{L}^{\widehat{\lambda * w}} \cong \mathcal{L}^{\widehat{\lambda}} \circ \mathbf{I}_{\tilde{w}}
$$

The proof appears in Section 2.3.1.

\footnotetext{
${ }^{14}$ Here by an action of a group $H$ on a category we mean a weak action, i.e., a homomorphism from $H$ to the group of isomorphism classes of auto-equivalences of the category. We expect that the action we construct can be enhanced to a strong action, where for each $g \in H$ a functor $F_{g}$ is given and isomorphisms $F_{g h} \cong F_{g} \circ F_{h}$ are fixed and satisfy the natural compatibilities. We do not attempt to construct this richer structure in the present paper.
} 
Remarks 2.1.5. 1) One can define the action in a slightly different way: one could require that $(7)$ holds when $w$ decreases $\lambda$. Then the canonical generators would act by functors $\mathbf{I}_{\alpha}^{!}$rather than $\mathbf{I}_{\alpha}^{*}$ (see Section 2.2 .1 below). That choice would yield standard formulas for the action of the affine Hecke algebra which have origin in the action on Iwahori invariant functions on the Langlands dual $p$-adic group, or on the corresponding categories of constructible sheaves (see $[\mathrm{Lu}],[\mathrm{CG}]$ ). The present normalization yields more convenient formulas for the action on the derived categories of coherent sheaves, cf. [B].

2) It follows from Lemma 2.2.3.a below that the finite abelian subgroup $\Omega \subset{ }^{\Theta} B_{\text {aff }}^{\prime}$ acts by translation functors, which are exact functors on the abelian category $\mathrm{U}_{\hat{\chi}}^{\lambda}$. E.g. for $G=S L(2), \lambda=0$ and $\chi=0$ one can show that the non identity element in $\Omega \cong \mathbb{Z} / 2 \mathbb{Z}$ interchanges the trivial and the $(p-1)$ dimensional module.

Corollary 2.1.6. For any regular integral $\lambda$ there exists an (obviously unique) action $b \mapsto \mathbb{I}_{b}$ of $B_{\text {aff }}^{\prime}$ on $\mathrm{D}^{b}\left[\bmod _{c}^{\lambda}(\widetilde{\mathcal{D}})\right]$ satisfying the following requirements:

i) Assume that $\lambda$ lies in the fundamental alcove $A_{0}$, so that the isomorphism isom $_{\lambda}: W_{\text {aff }}^{\prime} \cong{ }^{\Theta} W_{\text {aff }}^{\prime}$ respects the Coxeter structure, and therefore induces an isomorphism of braid groups isom $_{\lambda}: B_{\text {aff }}^{\prime} \cong{ }^{\Theta} B_{\text {aff }}^{\prime}$. Then the equivalence $\mathrm{R}_{\widetilde{\mathcal{D}}_{\mathcal{B}, \lambda}}: \mathrm{D}^{b}\left[\bmod _{\lambda}^{c}(\widetilde{\mathcal{D}})\right] \rightarrow \mathrm{D}^{b}\left[\bmod _{\lambda}^{f g}(\mathrm{U})\right]$ intertwines the actions of $B_{\text {aff }}^{\prime}$ and ${ }^{\Theta} B_{\text {aff }}^{\prime}$.

ii) If $\lambda, \mu$ are two integral regular weights, then the equivalence Tens ${ }_{\mu, \lambda}$ commutes with the $B_{\text {aff }}^{\prime}$ action.

See Section 2.3.2 for a proof of the corollary and a brief discussion of the properties of this action.

\subsection{Translation and intertwining functors for $\mathfrak{g}$-modules and $\mathcal{D}$-modules}

2.2.1. Translation and intertwining functors on $\mathfrak{g}$-modules

For $\mu \in \Lambda$ we denote by $M \mapsto[M]_{\mu}$ the projection of the category of finitely generated $\mathfrak{g}$-modules with a locally finite action of $\mathfrak{Z} \mathrm{HC}$ to its direct summand $\bmod _{\mu}^{f g}(\mathrm{U}) \stackrel{\text { def }}{=} \bmod _{d \mu}^{f g}(\mathrm{U})$ (see Section 1.4.1 for notations). For $\mu, \nu \in \Lambda$ the translation functor $T_{\nu}^{\mu}: \bmod _{\nu}^{f g}(\mathrm{U}) \rightarrow \bmod _{\mu}^{f g}(\mathrm{U})$ is defined by (see 1.1.1 for the representations $V_{\eta}$ and [BMR, Section 6] for more details)

$$
T_{\nu}^{\mu}(M) \stackrel{\text { def }}{=}\left[V_{\mu-\nu} \otimes M\right]_{\mu} \text {. }
$$


It is obvious from the definition that

$$
T_{\nu}^{\mu}=T_{w \cdot \mu}^{w \cdot \nu}, \quad w \in W_{\mathrm{aff}}^{\prime} .
$$

In particular, for $\lambda \in \Theta$ and $w \in{ }^{\Theta} W_{\text {aff }}^{\prime}$ the functor $T_{\lambda}^{\lambda * w}$ is independent of the choice of $\lambda \in \Theta$, we denote this functor by $T_{w}$.

In the situation where $\mu$ is regular and $\nu$ lies on a codimension one face of the alcove of $\mu$ one says that $T_{\mu}^{\nu}$ is a down functor and $T_{\nu}^{\mu}$ is an up functor. The composition up o down is called a reflection functor or a wall crossing functor

$$
R_{\mu \mid \nu}: \bmod _{\mu}^{f g}(\mathrm{U}) \longrightarrow \bmod _{\mu}^{f g}(\mathrm{U}), \quad R_{\mu \mid \nu} \stackrel{\text { def }}{=} T_{\nu}^{\mu} \circ T_{\mu}^{\nu} .
$$

These functors are exact, and we use the same notation for the corresponding functors on the derived categories. It is easy to show that $T_{\mu}^{\nu}$ and $T_{\nu}^{\mu}$ are always adjoint (in either order), hence $R_{\mu \mid \nu}$ is self adjoint and there are canonical maps id $\rightarrow R_{\mu \mid \nu} \rightarrow$ id. Define functors $\mathbf{I}_{\mu \mid \nu}^{*}, \mathbf{I}_{\mu \mid \nu}^{!}$as follows:

$$
\mathbf{I}_{\mu \mid \nu}^{!}=\operatorname{cone}\left(R_{\mu \mid \nu} \rightarrow \mathrm{id}\right)[-1] \quad \text { and } \quad \mathbf{I}_{\mu \mid \nu}^{*}=\operatorname{cone}\left(\mathrm{id} \rightarrow R_{\mu \mid \nu}\right)
$$

here cone denotes the cone of a map between exact functors on the category of complexes, thus the expressions in the right hand sides define exact endofunctors of the categories of complexes. We use the same notations $\mathbf{I}_{\mu \mid \nu}^{!}$, $\mathbf{I}_{\mu \mid \nu}^{*}$ for the induced endofunctors of the derived category $\mathrm{D}\left(\bmod _{\mu}^{f g}(\mathrm{U})\right)$. It is clear that these functors preserve the subcategories $\mathrm{D}^{-}$and $\mathrm{D}^{b}$.

\subsubsection{Intertwining functors and $\mathcal{D}$-modules}

Let us start with some notation. Recall that for $\mu \in \Lambda$ we have a canonical isomorphism between $\mathcal{D}^{\mu} \stackrel{\text { def }}{=} \mathcal{O}_{\mu} \mathcal{D}$ and the specialization $\mathcal{D}^{d \mu}$ of $\widetilde{\mathcal{D}} \stackrel{\text { def }}{=} \widetilde{\mathcal{D}}_{\mathcal{B}}$ to the differential $d \mu \in \mathfrak{h}^{*}$.

For two characters $\mu^{\prime}, \mu^{\prime \prime} \in \Lambda$ define a functor $\mathbf{I}_{\mu^{\prime \prime} \mu^{\prime}}: \mathrm{D}^{-}\left[\bmod _{\mu^{\prime}}^{f g}(\mathrm{U})\right] \rightarrow$ $\mathrm{D}^{-}\left[\bmod _{\mu^{\prime \prime}}^{f g}(\mathrm{U})\right]$ by

$$
\mathbf{I}_{\mu^{\prime \prime} \mu^{\prime}}(M) \stackrel{\text { def }}{=} \mathrm{R} \Gamma_{\widetilde{\mathcal{D}}_{\mathcal{B}}, \mu^{\prime \prime}}\left[\mathcal{L}^{\mu^{\prime}} M \otimes_{\mathcal{O}_{\mathcal{B}}} \mathcal{O}_{\mu^{\prime \prime}-\mu^{\prime}}\right]
$$

In the case when the differentials are regular in $\mathfrak{h}^{*}$ (i.e., characters are $\left(W_{\text {aff }}, \bullet\right)$-regular $), \mathbf{I}_{\mu^{\prime \prime} \mu^{\prime}}$ is an equivalence and one has $\mathbf{I}_{\mu^{\prime \prime} \mu^{\prime}} \circ \mathbf{I}_{\mu^{\prime} \mu} \cong \mathbf{I}_{\mu^{\prime \prime} \mu}$ and $\mathbf{I}_{\mu \mu}=$ id. In the case when $\Gamma\left(\mathcal{D}^{\mu^{\prime}}\right)=\Gamma\left(\mathcal{D}^{\mu^{\prime \prime}}\right)$ (this is equivalent to $d \mu^{\prime \prime} \in W \bullet d \mu^{\prime}$, i.e., to $\left.\mu^{\prime \prime} \in W_{\text {aff }}^{\prime} \bullet \mu^{\prime}\right)$, the categories $\mathrm{D}^{b}\left[\bmod _{\mu^{\prime}}^{f g}(\mathrm{U})\right]$ and $\mathrm{D}^{b}\left[\bmod _{\mu^{\prime \prime}}^{f g}(\mathrm{U})\right]$ coincide and $\mathbf{I}_{\mu^{\prime \prime} \mu^{\prime}}$ is an autoequivalence. 
LEMMA 2.2.3. Let $\mu, \nu \in \Lambda$ be characters such that $\nu$ is in the closure of the facet of $\mu$, and let $\mathcal{M} \in \mathrm{D}\left[\bmod _{\mu}^{c}(\widetilde{\mathcal{D}})\right]$ and $\mathcal{N} \in \mathrm{D}\left[\bmod _{\nu}^{c}(\widetilde{\mathcal{D}})\right]$.

(a) ("Down") We have a functorial isomorphism $T_{\mu}^{\nu}\left(\mathrm{R}_{\widetilde{\mathcal{D}}_{\mathcal{B}}, \mu} \mathcal{M}\right) \cong$ $\mathrm{R} \Gamma_{\widetilde{\mathcal{D}}_{\mathcal{B}}, \nu}\left(\mathcal{M} \otimes_{\mathcal{O}_{\mathcal{B}}} \mathcal{O}_{\nu-\mu}\right)$.

(b) ( $U p ")$ Assume also that $\mu$ is regular and $\nu$ lies in the (codimension one) face $H$ of the $\mu$-alcove. Let $\dot{\mu}$ denote the reflection of $\mu$ in the wall $H$. If $\dot{\mu}<\mu$, then there is an exact triangle

$$
\mathrm{R} \Gamma_{\widetilde{\mathcal{D}}_{\mathcal{B}, \mu}, \dot{\mathcal{N}}}\left(\mathcal{N} \otimes_{\mathcal{O}} \mathcal{O}_{\dot{\mu}-\nu}\right) \longrightarrow T_{\nu}^{\mu}\left(\mathrm{R} \Gamma_{\widetilde{\mathcal{D}}_{\mathcal{B}}, \nu} \mathcal{N}\right) \longrightarrow \mathrm{R} \Gamma_{\widetilde{\mathcal{D}}_{\mathcal{B}}, \mu}\left(\mathcal{N} \otimes_{\mathcal{O}} \mathcal{O}_{\mu-\nu}\right)
$$

(c) Keep the assumptions of $(b)$; if $\dot{\mu}<\mu$ then we have natural isomorphisms $\mathbf{I}_{\nu \mid \mu}^{!} \cong \mathbf{I}_{\mu \mu}$ and $\mathbf{I}_{\nu \mid \mu}^{*} \cong \mathbf{I}_{\mu \mu}$.

Proof. Claims (a) and (b) are in [BMR, Lemma 6.1.2]. For the reader's convenience we recall that they follow, respectively, from an isomorphism of sheaves of $\mathfrak{g}$-modules on $\mathcal{B}$ :

$$
T_{\mu}^{\nu}(\mathcal{F}) \cong \mathcal{F} \otimes \mathcal{O}_{\nu-\mu}
$$

for $\mathcal{F} \in \bmod _{\mu}^{c}(\widetilde{\mathcal{D}})$; and from the short exact sequence of sheaves of $\mathfrak{g} \bmod$ ules:

$$
0 \longrightarrow \mathcal{F} \otimes \mathcal{O}_{\mu-\nu} \longrightarrow\left[\mathcal{F} \otimes V_{\mu-\nu}\right]_{\mu} \longrightarrow \mathcal{F} \otimes \mathcal{O}_{\mu-\nu} \longrightarrow 0
$$

where $\mathcal{F} \in \bmod _{\nu}^{c}(\widetilde{\mathcal{D}}) ; \mu, \nu$ are as in (b) and $\dot{\mu}<\mu$ (cf. [BMR], Section 6.1.1). Here we have used the same notation $T_{\mu}^{\nu}, \mathcal{F} \mapsto \mathcal{F}_{\mu}$ for the functors on the categories of sheaves of $\mathfrak{g}$-modules as for the corresponding functors on the categories of $\mathfrak{g}$-modules, which were introduced earlier. The functors for sheaves are obtained by applying the corresponding functors for $\mathfrak{g}$-modules to the $\mathfrak{g}$-module of sections on each open subset.

It remains to prove (c). Assume first that $\dot{\mu}<\mu$; fix $M \in \mathrm{D}^{b}\left[\bmod _{\mu}^{f g}(\mathrm{U})\right]$, and set $\mathcal{M}=\mathcal{L}^{\mu} M$. We can represent $\mathcal{M}$ as a bounded complex $C_{\mathcal{M}}$ such that all $C_{\mathcal{M}}^{i} \in \bmod _{\mu}^{c}(\widetilde{\mathcal{D}})$ are $\Gamma$-acyclic where $\Gamma$ is the functor of global sections. Part (a) shows that the sheaves $C_{\mathcal{M}}^{i} \otimes \mathcal{O}_{\nu-\mu}$ are also $\Gamma$-acyclic. Hence the same is true for the sheaf $C_{\mathcal{M}}^{i} \otimes \mathcal{O}_{\nu-\mu} \otimes V_{\mu-\nu}$ and for its direct summand $\left[C_{\mathcal{M}}^{i} \otimes \mathcal{O}_{\nu-\mu} \otimes V_{\mu-\nu}\right]_{\mu}$. Using the above short exact sequence we see that the complex of sheaves of $\mathfrak{g}$ modules $C_{\mathcal{M}} \otimes \mathcal{O}_{\mu-\mu}$ is quasiisomorphic to the following complex of $\Gamma$-acyclic sheaves of $\mathfrak{g}$-modules:

$$
\operatorname{cone}\left(\left[C_{\mathcal{M}}^{\bullet} \otimes \mathcal{O}_{\nu-\mu} \otimes V_{\mu-\nu}\right]_{\mu} \rightarrow C_{\mathcal{M}}^{\bullet}\right)[-1] .
$$


Applying the functor of global sections to this complex we get the first isomorphism in (c); the second one is established in a similar way.

Corollary 2.2.4. Assume that $\mu$ is regular and $\nu$ is on a codimension one face of the alcove of $\mu$. Then $\mathbf{I}_{\mu \mid \nu}^{*}$ and $\mathbf{I}_{\mu \mid \nu}^{!}$are mutually inverse equivalences.

\subsubsection{Translation functors for coherent sheaves}

We now explain how to express the translation functors $T_{\mu}^{\nu}$ for some pairs $\mu, \nu \in \Lambda$ in terms of coherent sheaves. Consider a partial flag variety $\mathcal{P}$ and $\lambda \in \mathfrak{h}^{*}$ such that $\lambda$ is $\mathcal{P}$-regular and $\mathcal{P}$-unramified, so that the equivalences of Theorem 1.5.1.c apply for any $\chi$ with $(\chi, \lambda) \in \mathcal{Z}(\mathrm{U})$.

Recall that the choice of an equivalence in Theorem 1.5.1.c is determined by the choice of a splitting vector bundle for the Azumaya algebra $\widetilde{\mathcal{D}}_{\mathcal{P}}$ on the formal neighborhood of $\mathcal{Z}\left(\widetilde{\mathcal{D}}_{\mathcal{P}}\right) \times_{\mathcal{Z}(\mathrm{U})}(\chi, W \bullet \lambda)$ in $\mathcal{Z}\left(\widetilde{\mathcal{D}}_{\mathcal{P}}\right)$. Given such a bundle $\mathcal{M}$ we get an equivalence

$$
\begin{gathered}
\gamma_{\mathcal{M}}: \mathrm{D}^{b}\left[\mathcal{C o h}_{\mathcal{Z}\left(\widetilde{\mathcal{D}}_{\mathcal{P}}\right) \times \mathcal{Z}(\mathrm{U})}(\chi, W \bullet \lambda)\right. \\
\left.\left.\gamma_{\mathcal{M}}(\mathcal{F}) \stackrel{\text { def }}{=} \mathrm{R} \Gamma_{\widetilde{\mathcal{D}}_{\mathcal{P}, \lambda}}\left[\mathcal{M} \otimes_{\mathcal{P}}\right)\right)\right] \stackrel{\cong}{\cong} \mathrm{D}^{b}\left[\bmod _{\lambda, \chi} f g(\mathrm{U})\right] \\
\left.\mathcal{\mathcal { D }} \widetilde{\mathcal{P}}_{\mathcal{P}}\right)
\end{gathered}
$$

The splitting bundle $\mathcal{M}$ is unique up to tensoring with a line bundle.

We now assume that $\lambda \in \mathfrak{h}_{\mathbb{F}_{p}}^{*}$ is integral. Then the formal neighborhood of $\mathcal{Z}(\widetilde{\mathcal{D}}) \times \mathcal{Z}(\mathrm{U})(\chi, W \bullet \lambda)$ can be identified with the formal neighborhood of $\mathcal{P}_{\chi}^{(1)}=\mathcal{P}_{\chi, 0}{ }^{(1)}$ in $\widetilde{\mathfrak{g}}_{\mathcal{P}}^{*}$.

We have $\lambda=d \eta$ for some $\eta \in \Lambda$; moreover, since $\lambda$ is $\mathcal{P}$-regular and $\mathcal{P}$-unramified, we have $\operatorname{Stab}_{W}(\lambda)=W_{\mathcal{P}}$, thus we can (and will) assume that $\operatorname{Stab}_{W}(\eta)=W_{\mathcal{P}}$ (cf. Lemma 1.5.2.c).

Fixing such $\eta$ we get a splitting bundle $\mathcal{M}=\mathcal{M}_{\chi, \eta}^{\mathcal{P}}$ described in Remark 1.3.5. We let $\gamma_{\chi, \nu}^{\mathcal{P}}$ denote the corresponding equivalence.

In the next lemma we fix two parabolic subgroups $P \subset Q$, let $\pi_{\mathcal{Q}}^{\mathcal{P}}: \mathcal{P}=$ $G / P \rightarrow \mathcal{Q}=G / Q$ be the map between the partial flag varieties and $\widetilde{\pi}_{\mathcal{Q}}^{\mathcal{P}}$ be the natural map $\widetilde{\mathfrak{g}}_{\mathcal{P}}^{*} \rightarrow \widetilde{\mathfrak{g}}_{\mathcal{Q}}^{*}$.

Lemma. Let $\mu, \nu \in \Lambda$ be characters such that $\mu$ is $\mathcal{P}$-regular and $\mathcal{P}$ unramified, $\nu$ is $\mathcal{Q}$-regular and $\mathcal{Q}$-unramified and $\nu$ lies in the closure of the facet of $\mu$. 
For any nilpotent $\chi \in \mathfrak{g}^{*(1)}$ the down and up functors between $\bmod _{\mu, \chi}(\mathrm{U})$ and $\bmod _{\nu, \chi}(\mathrm{U})$ correspond under the equivalences with the derived categories of coherent sheaves to the push-forward and pull-back functors under $\tilde{\pi}_{\mathcal{Q}}^{\mathcal{P}}{ }^{(1)}$. In other words, we have natural isomorphisms:

$$
\begin{aligned}
& T_{\mu}^{\nu} \circ \gamma_{\chi, \mu}^{\mathcal{P}} \cong \gamma_{\chi, \nu}^{\mathcal{Q}} \circ \mathrm{R}\left(\tilde{\pi}_{\mathcal{Q}}^{\mathcal{P}}{ }^{(1)}\right)_{*} \quad \text { and } \\
& T_{\nu}^{\mu} \circ \gamma_{\chi, \nu}^{\mathcal{Q}} \cong \gamma_{\chi, \mu}^{\mathcal{P}} \circ L\left(\widetilde{\pi}_{\mathcal{Q}}^{\mathcal{P}}(1)\right)^{*} \cong \gamma_{\chi, \mu}^{\mathcal{P}} \circ L\left(\widetilde{\pi}_{\mathcal{Q}}^{\mathcal{P}}(1)\right)^{!}
\end{aligned}
$$

Proof. An isomorphism between $\left(T_{\mu}^{\nu} \circ \gamma_{\chi, \mu}^{\mathcal{P}}\right) \mathcal{F} \stackrel{\text { def }}{=} T_{\mu}^{\nu}\left[\mathrm{R} \Gamma\left(\mathcal{M}_{\chi, \mu}^{\mathcal{P}} \otimes_{\mathcal{O}_{\mathcal{Z}\left(\widetilde{\mathcal{D}}_{\mathcal{P}}\right)}}\right.\right.$ $\mathcal{F})]$ and $\left.\gamma_{\chi, \nu}^{\mathcal{Q}} \tilde{\pi}_{*}{ }^{(1)} \mathcal{F}=\operatorname{R} \Gamma\left(\mathcal{M}_{\chi, \nu}^{\mathcal{Q}} \otimes_{\mathcal{O}_{\mathcal{Z}\left(\widetilde{\mathcal{D}}_{\mathcal{Q}}\right)}} \tilde{\pi}_{*}^{(1)} \mathcal{F}\right)\right]$ can be obtained as the following composition:

$$
\begin{aligned}
& T_{\mu}^{\nu}\left[\mathrm{R} \Gamma\left(\mathcal{M}_{\chi, \mu}^{\mathcal{P}} \otimes_{\mathcal{O}_{\mathcal{Z}\left(\tilde{\mathcal{D}}_{\mathcal{P}}\right)}} \mathcal{F}\right)\right] \cong T_{\mu}^{\nu}\left[\mathrm{R} \Gamma\left(\pi_{\mathcal{P}}^{\mathcal{B}}\right)^{*}\left(\mathcal{M}_{\chi, \mu}^{\mathcal{P}} \otimes_{\mathcal{O}_{\mathcal{Z}\left(\tilde{\mathcal{D}}_{\mathcal{P}}\right)}} \mathcal{F}\right)\right] \\
& \cong \operatorname{R} \Gamma\left[\mathcal{O}_{\mathcal{B}, \nu-\mu} \otimes_{\mathcal{O}_{\mathcal{B}}}\left(\pi_{\mathcal{P}}^{\mathcal{B}}\right)^{*}\left(\mathcal{M}_{\chi, \mu}^{\mathcal{P}} \otimes_{\mathcal{O}_{\mathcal{Z}\left(\widetilde{\mathcal{D}}_{\mathcal{P}}\right)}} \mathcal{F}\right)\right] \\
& \cong \operatorname{R} \Gamma\left[\left(\pi_{\mathcal{P}}^{\mathcal{B}}\right)^{*}\left(\mathcal{O}_{\mathcal{P}, \nu-\mu} \otimes_{\mathcal{O}_{\mathcal{P}}}\left(\mathcal{M}_{\chi, \mu}^{\mathcal{P}} \otimes_{\mathcal{O}_{\mathcal{Z}\left(\tilde{\mathcal{D}}_{\mathcal{P}}\right)}} \mathcal{F}\right)\right)\right] \\
& \cong \operatorname{R\Gamma }\left[\left(\pi_{\mathcal{P}}^{\mathcal{B}}\right)^{*}\left(\mathcal{M}_{\chi, \nu}^{\mathcal{P}} \otimes_{\mathcal{O}_{\mathcal{Z}\left(\tilde{\mathcal{D}}_{\mathcal{P}}\right)}} \mathcal{F}\right)\right] \cong \mathrm{R} \Gamma\left(\mathcal{M}_{\chi, \nu}^{\mathcal{P}} \otimes_{\mathcal{O}_{\mathcal{Z}\left(\tilde{\mathcal{D}}_{\mathcal{P}}\right)}} \mathcal{F}\right)
\end{aligned}
$$

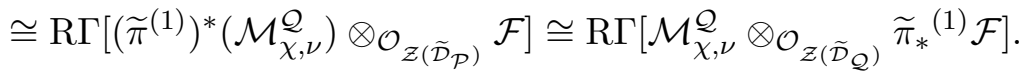

Here the key step is the second isomorphism, which is provided by Lemma 2.2.3.a. The first one is clear from the projection formula and the well-known fact that $\mathrm{R}\left(\pi_{\mathcal{P}}^{\mathcal{B}}\right)_{*} \mathcal{O}=\mathcal{O}$. The third isomorphism is a triviality (notice that $\nu-\mu$ is a character of the Levi of $P$, so $\mathcal{O}_{\mathcal{P}, \nu-\mu}$ is defined). The fourth one follows from $\mathcal{M}_{\chi, \nu}^{\mathcal{P}}=\mathcal{O}_{\mathcal{P}, \nu-\mu} \otimes_{\mathcal{O}_{\mathcal{P}}} \mathcal{M}_{\chi, \mu}^{\mathcal{P}}$ which is clear from the construction of these bundles in Remark 1.3.5. The fifth one again follows from $\mathrm{R}\left(\pi_{\mathcal{P}}^{\mathcal{B}}\right)_{*} \mathcal{O}=\mathcal{O}$. The sixth one is obtained by substituting (3) from Remark 1.3.5. The last one is the projection formula.

This proves the first isomorphism in the lemma. The second isomorphism follows since $T_{\mu}^{\nu}$ is both left and right adjoint to $T_{\nu}^{\mu}$, while $L \tilde{\pi}^{*}$ is the left adjoint of $\mathrm{R} \widetilde{\pi}_{*}$ and $L \tilde{\pi}^{!}$is the right adjoint of $\mathrm{R} \widetilde{\pi}_{*}$.

Remarks. 1) A variation of a well-known argument [BeG, 2.5] can be used to show that the functor $T_{\mu}^{\nu}$ sends the full subcategory $\bmod \left(\mathrm{U}^{\mu}\right) \subset$ $\bmod _{\mu}(\mathrm{U})$ to $\bmod \left(\mathrm{U}^{\nu}\right) \subset \bmod _{\nu}(\mathrm{U})$ and identifies $\bmod \left(\mathrm{U}^{\nu}\right)$ with a Serre quotient category of $\bmod \left(\mathrm{U}^{\mu}\right)$. It follows that $T_{\mu}^{\nu}$ sends an irreducible object to zero or to an irreducible object, and that every irreducible object in $\bmod \left(\mathrm{U}_{\chi}^{\nu}\right)$ is isomorphic to $T_{\mu}^{\nu}(L)$ for a unique irreducible object in $\bmod \left(\mathrm{U}_{\chi}^{\mu}\right)$. 
By adjunction, the functor $T_{\nu}^{\mu}$ sends the projective cover of an irreducible object in $\bmod _{\nu, \chi}(\mathrm{U})$ to the projective cover of the corresponding irreducible in $\bmod _{\mu, \chi}(\mathrm{U})$. (Here the projective cover is understood to be a pro-object in $\bmod _{\nu, \chi}(\mathrm{U})$.)

Together with the previous lemma this provides an effective tool for computation of coherent sheaves corresponding to some $\mathfrak{g}$ modules of interest.

2) The isomorphism $L \widetilde{\pi}^{*} \cong L \widetilde{\pi}^{!}$which was just deduced from the properties of translation functors also follows easily from the fact that $\widetilde{\mathfrak{g}}_{\mathcal{P}}^{*}$ and $\widetilde{\mathfrak{g}}_{\mathcal{Q}}^{*}$ have trivial canonical classes and their dimensions are equal.

\subsubsection{Composition of translation functors}

We need some more information on the behavior of translation functors. Recall the functors ${ }^{(15)} \mathrm{R} \Gamma: \mathrm{D}^{b}\left[\bmod ^{c}(\widetilde{\mathcal{D}})\right] \rightarrow \mathrm{D}^{b}\left[\bmod ^{f g}(\mathrm{U})\right], \mathcal{L}:$ $\mathrm{D}^{b}\left[\bmod ^{f g}(\mathrm{U})\right] \rightarrow \mathrm{D}^{b}\left[\bmod ^{c}(\widetilde{\mathcal{D}})\right], \mathcal{L}: M \rightarrow \widetilde{\mathcal{D}} \stackrel{\mathrm{L}}{\otimes_{\mathrm{U}}} M$. The functor $\mathrm{R} \Gamma$ can be "upgraded" to the derived functor of global sections $\mathrm{R} \widetilde{\Gamma}: \mathrm{D}^{b}\left[\bmod ^{c}(\widetilde{\mathcal{D}})\right] \rightarrow$ $\mathrm{D}^{b}\left[\bmod ^{f g}(\widetilde{\mathrm{U}})\right]$.

For $\lambda \in \mathfrak{h}^{*}$ let $\bmod _{\lambda}(\widetilde{\mathrm{U}}) \subset \bmod (\widetilde{\mathrm{U}})$ be the subcategory of modules where the central subalgebra $S(\mathfrak{h}) \subset \widetilde{\mathrm{U}}$ acts through the quotient by a power of the maximal ideal defined by $\lambda$. Then $\mathrm{R} \widetilde{\Gamma}$ restricts to $\mathrm{R} \widetilde{\Gamma}_{\lambda}$ : $\mathrm{D}^{b}\left[\bmod _{\lambda}(\widetilde{\mathcal{D}})\right] \rightarrow \mathrm{D}^{b}\left[\bmod _{\lambda}(\widetilde{\mathrm{U}})\right]$. Let also $\mathrm{U}^{\widehat{\lambda}}$, $\widetilde{\mathrm{U}}^{\widehat{\lambda}}$ denote the completions of U, $\widetilde{\mathrm{U}}$ at the corresponding character of $\mathfrak{Z}_{\mathrm{HC}}, S(\mathfrak{h})$ respectively; thus $\bmod _{\lambda}(\mathrm{U}), \bmod _{\lambda}(\widetilde{\mathrm{U}})$ are identified with full subcategories in, respectively, $\bmod \left(\mathrm{U}^{\widehat{\lambda}}\right), \widetilde{\bmod }\left(\mathrm{U}^{\widehat{\lambda}}\right)$.

LEMmA. For an integral $\lambda \in \mathfrak{h}_{\mathbb{F}_{p}}^{*}$ we have canonical isomorphisms

$$
\mathrm{R} \widetilde{\Gamma} \circ \mathcal{L} \cong \operatorname{Ind}_{\mathrm{U}}^{\widetilde{\mathrm{U}}}, \quad \mathrm{R} \widetilde{\Gamma} \circ \mathcal{L}^{\widehat{\lambda}} \cong \operatorname{Ind}_{\mathrm{U}^{\widehat{\lambda}}}^{\widetilde{\mathrm{U}}^{\widehat{\lambda}}}
$$

Proof. The isomorphisms of functors on derived categories follow from the corresponding isomorphisms of functors on the categories of bounded complexes of projective objects. The latter are established directly, cf. [BMR], Corollary 3.4.2.

\footnotetext{
${ }^{15}$ Notice that these are not the functors used above to get the localization theorem: the latter are direct summands of the former.
} 
Proposition. If $\mu$ lies in the closure of the $\lambda$ facet, and $\nu$ lies in the closure of the $\mu$-facet, then there is a canonical isomorphism of functors $T_{\mu}^{\nu} \circ T_{\lambda}^{\mu} \cong T_{\lambda}^{\nu}$ and also $T_{\mu}^{\lambda} \circ T_{\nu}^{\mu} \cong T_{\nu}^{\lambda}$.

In particular, if $\mu$ and $\nu$ are in the same facet then $T_{\mu}^{\nu}$ and $T_{\nu}^{\mu}$ are inverse equivalences.

Proof. We construct the first isomorphism, the second one follows by adjointness. Lemma 2.2.3.a implies that

$$
T_{\mu}^{\nu} \circ T_{\lambda}^{\mu} \circ \mathrm{R} \Gamma_{\widetilde{\mathcal{D}}_{\mathcal{B}}, \lambda} \cong T_{\lambda}^{\nu} \circ \mathrm{R} \Gamma_{\widetilde{\mathcal{D}}_{\mathcal{B}}, \lambda}
$$

canonically. By Lemma 2.2.6 this implies that

$$
T_{\mu}^{\nu} \circ T_{\lambda}^{\mu} \circ \operatorname{Res}_{\mathrm{U}^{\widehat{\lambda}}}^{\widetilde{\mathrm{U}}^{\widehat{\lambda}}} \operatorname{Ind}_{\mathrm{U}^{\widehat{\lambda}}}^{\widetilde{\mathrm{U}}^{\widehat{\lambda}}} \cong T_{\lambda}^{\nu} \circ \operatorname{Res}_{\mathrm{U}^{\widehat{\lambda}}}^{\widetilde{\mathrm{U}}^{\widehat{\lambda}}} \operatorname{Ind}_{\mathrm{U}^{\widehat{\lambda}}}^{\widetilde{\mathrm{U}}^{\widehat{\lambda}}} .
$$

The assumption $p>h$ implies that $p$ does not divide $|W|$, thus the subspace of $W$ invariants in $S(\mathfrak{h})$ is canonically a direct summand as a module for $S(\mathfrak{h})^{W}$. Similarly, the completion $S(\mathfrak{h})^{\widehat{\lambda}}$ contains $\left(S(\mathfrak{h})^{W}\right)^{\widehat{\lambda}}$ as a canonical direct summand. Thus $T_{\mu}^{\nu} \circ T_{\lambda}^{\mu}, T_{\nu}^{\mu}$ are canonical direct summands in the left hand side (respectively, right hand side) of the last isomorphism. This yields a morphism $T_{\mu}^{\nu} \circ T_{\lambda}^{\mu} \rightarrow T_{\lambda}^{\nu}$ defined as a composition of the embedding of a direct summand with the last isomorphism and the projection to a direct summand.

Applying both $T_{\mu}^{\nu} \circ T_{\lambda}^{\mu}$ and $T_{\lambda}^{\nu}$ to the object $\mathrm{U}^{\lambda}=R \Gamma_{\widetilde{\mathcal{D}}_{\mathcal{B}, \lambda}}\left(\mathcal{D}^{\lambda}\right)$ we get isomorphic objects by (8). It is straightforward to check that this isomorphism coincides with the map coming from the morphism of functors constructed in the previous paragraph. Since both functors are exact, and $\mathrm{U}^{\lambda}$ generates $\bmod ^{\lambda}(\mathrm{U})$ under extensions and taking cokernels, the desired isomorphism follows.

Corollary 2.2.7. Assume that $\mu$ is regular, and $\nu, \nu^{\prime}$ lie on the same codimension one face of the alcove of $\mu$. Then we have a canonical isomorphism $R_{\mu \mid \nu} \cong R_{\mu \mid \nu^{\prime}}$.

The corollary allows one to define a reflection functor $R_{\alpha}$ : $\mathrm{D}^{b}\left[\bmod _{\lambda}^{f g}(\mathrm{U})\right] \rightarrow \mathrm{D}^{b}\left[\bmod _{\lambda}^{f g}(\mathrm{U})\right]$ for any $\alpha \in{ }^{\Theta} I_{\text {aff }}$, as follows. Recall that $\alpha \in{ }^{\Theta} I_{\text {aff }}$ is an orbit of $W_{\text {aff }}^{\prime}$ on the set of pairs $(\lambda, F)$ with $\lambda \in \Theta$ and $F$ a face of the $\lambda$-alcove. Choose a representative $(\lambda, F)$ of $\alpha$ and a character $\nu \in F$. We then set $R_{\alpha}=R_{\lambda \mid \nu}$. The corollary shows that $R_{\alpha}$ is independent of these choices. Similarly, we get functors $\mathbf{I}_{\alpha}^{*}, \mathbf{I}_{\alpha}^{!}, \alpha \in{ }^{\Theta} I_{\text {aff }}$. 
Proposition 2.2.8. For $\alpha \in{ }^{\Theta} I_{\text {aff }}$ and $\mu \in \Theta$ such that $\dot{\mu}=\mu * s_{\alpha}>\mu$ we have $\mathcal{L}^{\widehat{\mu}} \circ \mathbf{I}_{\alpha}^{*} \cong \mathcal{L}^{\hat{\dot{\mu}}}$.

Proof. This is a restatement of Lemma 2.2.3.c.

\subsection{Affine braid group actions: proofs}

\subsubsection{Proof of Theorem 2.1.4}

It suffices to check that:

(1) If $\omega \in{ }^{\Theta} B_{\text {aff }}^{\prime}, \ell(\omega)=0$, then for all $\lambda \in \Theta$ we have a canonical isomorphism $\mathcal{L}^{\widehat{\lambda * \omega}} \cong \mathcal{L}^{\widehat{\lambda}} \circ T_{\omega}$ (see the first paragraph of Section 2.2.1 for the notation).

(2) Let $\alpha \in{ }^{\Theta} I_{\text {aff }}$ and let $s=s_{\alpha} \in{ }^{\Theta} B_{\text {aff }}$ be the corresponding simple reflection. If $s$ increases $\lambda \in \Theta$, then $\mathcal{L}^{\widehat{\lambda * s}} \cong \mathcal{L}^{\widehat{\lambda}} \circ \mathbf{I}_{\alpha}^{*}$.

(3) The map $\tilde{\omega} \mapsto T_{\omega}, \widetilde{s}_{\alpha} \mapsto \mathbf{I}_{\alpha}^{*}$ extends to an action of ${ }^{\Theta} B_{\text {aff }}^{\prime}$.

We claim that (3) follows from (1) and (2). Indeed, for $w \in{ }^{\Theta} W_{\text {aff }}$ it is easy to find an element $\lambda \in \Theta$, such that $w$ increases $\lambda$. By induction on $\ell(w)$ it follows from (1) and (2) that for any decomposition $w=\omega s_{\alpha_{1}} \cdots s_{\alpha_{\ell(w)}}$ where $\ell(\omega)=0$ and $\alpha_{i} \in{ }^{\Theta} I_{\text {aff }}$ we have

$$
\mathcal{L}^{\widehat{\mu}} \cong \mathcal{L}^{\widehat{\lambda}} \circ T_{\omega} \circ \mathbf{I}_{\alpha_{1}}^{*} \circ \cdots \circ \mathbf{I}_{\alpha_{\ell(w)}}^{*}
$$

where $\mu=\lambda * \omega * s_{\alpha_{1}} * \cdots * s_{\alpha_{\ell(w)}}$. This shows that the composition of functors on the right hand side does not depend on the reduced decomposition of $w$, which implies (3).

It remains to check (1) and (2). In fact, (1) is immediate from Lemma 2.2.3.a, while (2) follows from Lemma 2.2.3.c.

2.3.2. The action of $B_{\text {aff }}^{\prime}$ on $\widetilde{\mathcal{D}}$ modules: proof of Corollary 2.1.6

It suffices to check the following: if $\lambda, \mu$ lie in the fundamental alcove $A_{0}$, then the translation functor $T_{\lambda}^{\mu}$ intertwines the action of ${ }^{\Theta(\lambda)} B_{\text {aff }}^{\prime}$ with the action of ${ }^{\Theta(\mu)} B_{\text {aff }}^{\prime}$; here we have identified ${ }^{\Theta(\lambda)} B_{\text {aff }}^{\prime}$ with ${ }^{\Theta(\mu)} B_{\text {aff }}^{\prime}$ by means of the composed isomorphism:

$$
{ }^{(}{ }^{(\lambda)} B_{\mathrm{aff}}^{\prime} \stackrel{\text { isom }_{\lambda}^{-1}}{\longrightarrow} B_{\mathrm{aff}}^{\prime} \stackrel{\text { isom }_{\mu}}{\longrightarrow} \Theta^{(\mu)} B_{\mathrm{aff}}^{\prime} .
$$

This compatibility follows from the definitions and Proposition 2.2.6. 
Remark. The action of $B_{\text {aff }}^{\prime}$ on $\mathrm{D}^{b}\left[\bmod _{\lambda}^{c}(\widetilde{\mathcal{D}})\right]$, and a closely related action on $\mathrm{D}^{b}\left[\mathcal{C} \mathrm{oh}\left(\widetilde{\mathfrak{g}}^{*}\right)\right], \mathrm{D}^{b}[\mathcal{C} \mathrm{oh}(\widetilde{\mathcal{N}})]$ will be discussed in more detail in a future publication. There we will provide a geometric description of the action of generators in terms of convolution with some explicit sheaves on $\left(\widetilde{\mathfrak{g}}^{*}\right)^{2}$, analogous to the one for the characteristic zero setting given in [BB2]. Also, we will show that the induced action on the $K$-groups of Springer fibers factors through the affine Weyl group and extends to an action on the equivariant $K$-groups, where it gives the action of the affine Hecke algebra on a standard module.

Here we state only a simple property of the action on $\mathrm{D}^{b}\left[\bmod _{\lambda}^{c}(\widetilde{\mathcal{D}})\right]$.

Proposition 2.3.3. Fix a regular $\lambda \in \Lambda$. For $\nu \in \Lambda^{+} \subset W_{\text {aff }}^{\prime}$ on $\mathrm{D}^{b}\left(\bmod _{\lambda}^{c}(\widetilde{\mathcal{D}})\right)$ the action of $\mathbb{I}_{\tilde{\nu}}$ is given by $\mathbb{I}_{\widetilde{\nu}}: \mathcal{F} \mapsto \mathcal{F} \otimes_{\mathcal{O}_{\mathcal{B}^{(1)}}} \mathcal{O}_{\nu}{ }^{(1)}$.

Proof. Pick $\lambda \in A_{0}$. Let $g_{\nu}$ be the image of $\nu \in \Lambda^{+} \subset W_{\text {aff }}^{\prime}$ under the isomorphism isom $\lambda$; thus $\lambda * g_{\nu}=\lambda+p \nu$ and $g_{\nu}$ increases $\lambda$. Then for $\mathcal{M} \in \bmod _{\lambda}^{c}(\widetilde{\mathcal{D}})$ we have $\mathbb{I}_{\widetilde{\nu}}(\mathcal{M})=\mathcal{L}\left(\mathbf{I}_{g_{\nu}}(R \Gamma(\mathcal{M}))\right.$ ) (cf. the characterizations of the actions in Corollary 2.1.6). On the other hand we have

$$
\mathcal{L}\left(\mathbf{I}_{g_{\nu}}(R \Gamma(\mathcal{M}))\right) \cong \operatorname{Tens}_{\lambda+p \nu, \lambda} \mathcal{L}(R \Gamma(\mathcal{M}))=\left(\mathcal{O}_{\mathcal{B}^{(1)}}\right)_{\nu} \otimes_{\mathcal{O}_{\mathcal{B}}(1)} \mathcal{M}
$$

where the first isomorphism is a particular case of (7) from Theorem 2.1.4, and the second one follows from the definition of $\operatorname{Tens}_{\lambda, \mu}$.

\section{§3. Dualities}

In this section we translate the duality operation on $\mathfrak{g}$-modules to $\mathcal{D}$ modules and coherent sheaves. This section is directly inspired by Lusztig [Lu, Part II, Remark] (see Remark 3.0.12 below).

\subsubsection{Homological duality}

For a $\mathfrak{g}$-module $M$ the dual $\mathbb{k}$ vector space $M^{*}$ carries a $\mathfrak{g}$ action, we denote the resulting $\mathfrak{g}$-module by $M^{\curlyvee}$. Thus we get a contravariant equivalence between finite dimensional $\mathrm{U}_{\hat{\chi}}^{\hat{\lambda}}$-modules and finite dimensional $\frac{\mathrm{U}^{\overline{\lambda^{\nu}}}}{\widehat{-\chi}}$ modules, which sends $M$ to $M^{\llcorner}$; here $\lambda^{\llcorner}=-w_{0}(\lambda)$ is the dual weight (recall that $w_{0} \in W$ is the long element). We use the same notation $M \mapsto M^{\sim}$ for the anti-equivalence of the derived categories induced by the exact functor on abelian categories. 
The aim of this section is to describe the localization of $M^{\sim}$ in terms of localization of $M$. We start by extending the duality to $\mathrm{D}^{b}\left[\bmod ^{f g}(\mathrm{U})\right]$. To this end recall the homological duality $\mathbb{D}_{\mathrm{U}}: M \mapsto \operatorname{RHom}(M, \mathrm{U})[\operatorname{dim} \mathfrak{g}]$, where the latter is understood to be an object in the derived category of right U-modules (which can, of course, be identified with left modules in a standard way).

In the following lemma we assume that $\mathfrak{g}$ is any finite dimensional unimodular Lie algebra (i.e. the adjoint action of $\mathfrak{g}$ on its top exterior power is trivial) over any field $k$ (not of characteristic 2 or 3 ).

Lemma. Let $M$ be a finite complex of finite dimensional $\mathfrak{g}$-modules. We have a canonical isomorphism in $\mathrm{D}^{b}[\bmod (\mathrm{U}(\mathfrak{g}))]: M^{\sim} \cong \mathbb{D}_{\mathrm{U}}(M)$.

Proof. Let $C^{\bullet}$ be the standard projective resolution for the trivial $\mathrm{U}(\mathfrak{g})$-module, thus $C^{i}=\Lambda^{-i}(\mathfrak{g}) \otimes \mathrm{U}(\mathfrak{g})$ with Koszul-Chevalley differential. Choosing an isomorphism $k \cong \Lambda^{\operatorname{dim} \mathfrak{g}}(\mathfrak{g})$ we get an isomorphism $\Lambda^{\operatorname{dim} \mathfrak{g}-d}(\mathfrak{g}) \cong \Lambda^{d}(\mathfrak{g})^{*}$, which yields an isomorphism of complexes of $\mathfrak{g}$ modules $\operatorname{Hom}_{\mathrm{U}}\left(C^{\bullet}, \mathrm{U}\right) \cong C^{\bullet}[-\operatorname{dim} \mathfrak{g}]$.

We have isomorphisms in $\mathrm{D}^{b}[\bmod (\mathrm{U}(\mathfrak{g}))]$ :

$$
\begin{aligned}
\mathbb{D}_{\mathrm{U}}(M) & \cong \operatorname{Hom}_{\mathrm{U}}\left(M \otimes C^{\bullet}, \mathrm{U}[\operatorname{dim} \mathfrak{g}]\right) \cong M^{\llcorner} \otimes \operatorname{Hom}_{\mathrm{U}}\left(C^{\bullet}, \mathrm{U}[\operatorname{dim} \mathfrak{g}]\right) \\
& \cong M^{\llcorner} \otimes C^{\bullet} \cong M^{\llcorner},
\end{aligned}
$$

which yields the result.

3.0.2. Duality for $\widetilde{\mathcal{D}}$ modules

We define the homological duality for $\widetilde{\mathcal{D}}$ modules, $\mathbb{D}_{D}: \mathrm{D}^{b}\left[\bmod ^{c}(\widetilde{\mathcal{D}})\right] \rightarrow$ $\mathrm{D}^{b}\left[\bmod ^{c}\left(\widetilde{\mathcal{D}}^{o p}\right)\right], \mathbb{D}_{D}(\mathcal{M})=\mathrm{R} \mathcal{H o m}(M, \widetilde{\mathcal{D}})[\operatorname{dim} \mathfrak{g}]$.

Recall the functors $\mathrm{R} \Gamma: \mathrm{D}^{b}\left[\bmod ^{c}(\widetilde{\mathcal{D}})\right] \rightarrow \mathrm{D}^{b}\left[\bmod ^{f g}(\mathrm{U})\right], \quad \mathcal{L}:$ $\mathrm{D}^{b}\left[\bmod ^{f g}(\mathrm{U})\right] \rightarrow \mathrm{D}^{b}\left[\bmod ^{c}(\widetilde{\mathcal{D}})\right], \mathcal{L}: M \rightarrow \widetilde{\mathcal{D}} \stackrel{\mathrm{L}}{\otimes} \mathrm{U} M$. We use the same notation for similarly defined functors between derived categories of right $\mathcal{D}$-modules and right $\mathrm{U}$-modules.

LEMma 3.0.3. We have canonical isomorphisms of functors $\mathcal{L} \circ \mathbb{D}_{\mathrm{U}} \cong$ $\mathbb{D}_{D} \circ \mathcal{L}, \mathrm{R} \Gamma \circ \mathbb{D}_{D} \cong \mathbb{D}_{\mathrm{U}} \circ \mathrm{R} \Gamma$.

Proof. It is clear that we have natural isomorphisms

$$
\mathcal{L} \circ \mathbb{D}_{\mathrm{U}}(\mathrm{U}) \cong \mathcal{L}(\mathrm{U}[\operatorname{dim} \mathfrak{g}]) \cong \widetilde{\mathcal{D}}[\operatorname{dim} \mathfrak{g}] \cong \mathbb{D}_{D}(\widetilde{\mathcal{D}}) \cong \mathbb{D}_{D}(\mathcal{L}(\mathrm{U}))
$$


moreover, it is easy to see that this isomorphism is compatible with the action of $U=\operatorname{End}_{U}(U)$. Thus we get an isomorphism of the two functors on the category of bounded complexes of finitely generated projective U-modules induced, respectively, by $\mathcal{L} \circ \mathbb{D}_{\mathrm{U}}$ and $\mathbb{D}_{D} \circ \mathcal{L}$. The ensuing isomorphism of functors on the derived category is the first of the two desired isomorphisms.

The functor $\mathcal{L}$ is left adjoint to $\mathrm{R} \Gamma$. It has been shown in the first chapter (see Corollary 1.8.2.c) that both categories $\mathrm{D}^{b}\left[\bmod ^{f g}(\mathrm{U})\right]$ and $\mathrm{D}^{b}\left[\bmod ^{c}(\widetilde{\mathcal{D}})\right]$ are Calabi-Yau categories over $\mathcal{O}\left(\mathfrak{g}^{*(1)}\right)$. It follows that $\mathcal{L}$ is also right adjoint to $\mathrm{R} \Gamma$. In view of the two adjunctions the second isomorphism follows from the first one.

\subsubsection{Right $\mathcal{D}$-modules as twisted left $\mathcal{D}$-modules}

Let $X$ be a smooth $d$-dimensional variety over the field $\mathbb{k}$, and let $\Omega_{X}$ be the sheaf of volume forms. We have the Lie derivative action of vector fields on $\Omega_{X}, \xi: \omega \rightarrow \operatorname{Lie}_{\xi}(\omega)=d\left(i_{\xi} \omega\right)$ (the term $i_{\xi} d$ vanishes on top forms).

Lemma. a) Let $\mathcal{M}$ be a sheaf of $\mathcal{D}_{X}$ modules. The action of vector fields $\xi: \omega \otimes \sigma \mapsto-\operatorname{Lie}_{\xi}(\omega) \otimes \sigma-\omega \otimes \xi(\sigma)$ extends to an action of $\mathcal{D}_{X}^{o p}$ on $\Omega_{X} \otimes_{\mathcal{O}_{X}} \mathcal{M}$. The resulting functor $\mathcal{M} \mapsto \mathcal{M} \otimes \Omega_{X}$ provides a (covariant) equivalence between the categories of left and right $\mathcal{D}_{X}$ modules.

b) The action of $\mathcal{D}_{X}^{o p}$ on $\Omega_{X}$ (the case $\mathcal{M}=\mathcal{O}_{X}$ in (a)), identifies $\mathcal{D}_{X}^{\text {op }}$ with the algebra ${ }^{\Omega_{X}} \mathcal{D}_{X}=\Omega_{X} \otimes \mathcal{D}_{X} \otimes \Omega_{X}{ }^{-1}$ of differential operators on the line bundle $\Omega_{X}$.

c) Let $\omega$ be a nowhere vanishing section of $\Omega_{X}$. Then there exists an isomorphism $I_{\omega}: \mathcal{D}_{X} \cong \mathcal{D}_{X}^{o p}$, such that the actions of $\mathcal{D}_{X}$ and $\mathcal{D}_{X}^{o p}$ on $\mathcal{D}_{X} \otimes \Omega_{X}=\Omega_{X} \otimes \mathcal{D}_{X}^{o p}$ are related by $d(\omega \otimes 1)=\omega \otimes I_{\omega}(d)$ for any section d of $\mathcal{D}_{X}$.

Proof. The proof is the same as in the characteristic zero case, cf., e.g., [Bo, Chapter VI.3].

\subsubsection{Duality and splitting bundles}

We now apply these general considerations to the case of the flag variety. In the remainder $\nu$ lies in $\Lambda=X^{*}(T)$ and $\chi \in \mathfrak{g}^{*(1)}$ is nilpotent. We will sometimes identify the formal neighborhood of $\mathcal{B}_{\chi}{ }^{(1)} \times\{d \nu\}$ in $\mathcal{Z}\left(\widetilde{\mathcal{D}}_{\mathcal{B}}\right)=$ $\widetilde{\mathfrak{g}}^{*(1)} \times_{\mathfrak{h}^{*(1)}} \mathfrak{h}^{*}$ with $\mathrm{FN}_{\widetilde{\mathfrak{g}}^{*(1)}}\left(\mathcal{B}_{\chi}{ }^{(1)}\right)$, we have such a canonical identification, since the Artin-Schreier map $\mathfrak{h}^{*} \rightarrow \mathfrak{h}^{*(1)}$ is etale. 
Recall a canonical choice of a splitting bundle $\mathcal{M}_{\chi}^{\nu}$ for the Azumaya algebra $\widetilde{\mathcal{D}}$ on the formal neighborhood of a Springer fiber $\mathcal{B}_{\chi}{ }^{(1)} \times\{d \nu\} \subset$ $\mathcal{Z}(\widetilde{\mathcal{D}})$, see 1.3.5. First, $\mathcal{M}_{\chi}^{-\rho}$ is a pull-back from a formal neighborhood of $(\chi,-\rho)$ in $\mathcal{Z}(U)=\mathfrak{g}^{*(1)} \times_{\mathfrak{h}^{*(1)} / W} \mathfrak{h} / W$, so it is trivial as a vector bundle. The general case is obtained by twisting, i.e., $\mathcal{M}_{\chi}^{\nu} \stackrel{\text { def }}{=} \mathcal{M}_{\chi}^{-\rho} \otimes_{\mathcal{O}_{\mathcal{B}}} \mathcal{O}_{\nu+\rho}$.

The above choice of the splitting bundles $\mathcal{M}_{\chi}^{\nu}$ is used to construct the equivalence of coherent sheaves and $\mathfrak{g}$-modules:

$$
\gamma_{\chi, \nu}(\mathcal{F}) \stackrel{\text { def }}{=} \mathrm{R} \Gamma\left(\mathcal{M}_{\chi}^{\nu} \otimes \mathcal{F}\right), \quad \mathcal{F} \in \mathrm{D}^{b}\left[\mathcal{C o h}_{\mathcal{B}_{\chi}}\left(\widetilde{\mathfrak{g}}^{*}\right)\right]
$$

LEMMA 3.0.6. a) We have a canonical isomorphism $\mathfrak{I}: \widetilde{\mathcal{D}} \cong \widetilde{\mathcal{D}}^{\text {op }}$ compatible with the standard isomorphism $\mathrm{U}^{o p} \cong \mathrm{U}$. Its restriction to the center coincides with the map induced by the involution $\iota$ of $\widetilde{\mathfrak{g}}^{*(1)} \times_{\mathfrak{h}^{*(1)}} \mathfrak{h}^{*}$ given by $\iota(\mathfrak{b}, x ; \nu)=(\mathfrak{b},-x ;-2 \rho-\nu)$.

b) There exists an isomorphism of $\widetilde{\mathcal{D}}$ modules

$$
\mathcal{M}_{\chi}^{-\rho+\nu} \cong \mathfrak{I}^{*} \mathbb{D}_{D}\left(\mathcal{M}_{-\chi}^{-\rho-\nu}\right)[-\operatorname{dim} \mathfrak{g}]
$$

compatible with the above isomorphism of algebras.

Proof. (a) The canonical line bundle on $G / J$ is the pull back of the canonical line bundle on $\mathcal{B}$. We have a well-known isomorphism $\Omega_{\mathcal{B}} \cong \mathcal{O}_{-2 \rho}$, thus the canonical line bundle of $G / J$ is $G$-equivariantly trivial. Thus a choice of a $G$-invariant nonzero section $\omega$ is unique up to scaling. By the previous lemma $\omega$ provides a (canonical) isomorphism $\mathcal{D}_{G / J}^{o p} \cong \mathcal{D}_{G / J}$, which leads to $\mathfrak{I}$ since $\widetilde{\mathcal{D}}$ is obtained from $\mathcal{D}_{G / J}$ by taking direct image to $\mathcal{B}$ and invariants with respect to $H$. The formula for the action on the center comes from the fact that $\omega$ transforms by the character $-2 \rho$ under the action of the abstract Cartan group $H$.

It remains to check (b). For $\nu=0$ the isomorphism follows from the fact that the Azumaya algebra $\left.\widetilde{\mathcal{D}}\right|_{\mathrm{FN}\left(\mathcal{B}_{\chi} \times\{\rho\}\right)}$ and its two splitting bundles $\mathcal{M}_{\chi}^{-\rho}$ and $\mathfrak{I}^{*} \mathbb{D}_{D}\left(\mathcal{M}_{-\chi}^{-\rho}\right)[-\operatorname{dim} \mathfrak{g}] \cong \mathfrak{I}^{*}\left(\mathcal{M}_{-\chi}^{-\rho}\right)^{*}$ (where the last $*$ denotes the dual vector bundle) are pull-backs of the corresponding structures on $\mathrm{FN}_{\mathfrak{g}^{*(1)} \times_{\mathfrak{h}^{*(1)} / W} \mathfrak{h}^{*} / W}(\chi,-\rho)$, while any two splitting bundles for an Azumaya algebra over the ring of Taylor series are isomorphic.

For a general $\nu$ the isomorphism follows from $\mathcal{M}_{\chi}^{-\rho+\nu}=\mathcal{O}_{\nu} \otimes_{\mathcal{O}_{\mathcal{B}}} \mathcal{M}_{\chi}^{-\rho}$ (see 3.0.5). 


\subsubsection{Grothendieck-Serre duality $\mathbb{D}_{\mathcal{O}}$}

The canonical line bundle for $\widetilde{\mathfrak{g}}^{*}$ is trivial, and then the same is true for $\widetilde{\mathfrak{g}}^{*(1)} \times_{\mathfrak{h}^{*(1)}} \mathfrak{h}^{*}$. Therefore, the Grothendieck-Serre duality functor for $\mathrm{D}^{b}\left[\mathcal{C}\right.$ oh $\left.\left(\widetilde{\mathfrak{g}}^{*(1)} \times_{\mathfrak{h}^{*}(1)} \mathfrak{h}^{*}\right)\right]$ is given by $\mathbb{D}_{\mathcal{O}}: \mathcal{F} \mapsto \operatorname{R\mathcal {Hom}}(\mathcal{F}, \mathcal{O})[\operatorname{dim} \mathfrak{g}]$. We let $\sigma$ denote the involution of $\widetilde{\mathfrak{g}}^{*}$ sending $(\mathfrak{b}, \chi)$ to $(\mathfrak{b},-\chi)$.

Corollary 3.0.8. For $\mathcal{F} \in \mathrm{D}^{b}\left[\mathcal{C o h}_{\mathcal{B}_{\chi}}\left(\widetilde{\mathfrak{g}}^{*}\right)\right]$ we have a canonical isomorphism

$$
\mathbb{D}_{D}\left(\mathcal{F} \otimes \mathcal{M}_{\chi}^{-\rho+\nu}\right) \cong \sigma^{*} \mathbb{D}_{\mathcal{O}}(\mathcal{F}) \otimes \mathcal{M}_{-\chi}^{-\rho-\nu}
$$

Proof. For a coherent sheaf $\mathcal{F}$ on $\widetilde{\mathfrak{g}}^{*(1)} \times_{\mathfrak{h}^{*(1)}} \mathfrak{h}^{*}$ and an object $\mathcal{M} \in$ $\bmod ^{c}(\widetilde{\mathcal{D}})$ the tensor product sheaf $\mathcal{F} \otimes \mathcal{O} \mathcal{M}$ on $\widetilde{\mathfrak{g}}^{*(1)} \times_{\mathfrak{h}^{*(1)}} \mathfrak{h}^{*}$ carries a $\widetilde{\mathcal{D}}$ action. It is obvious that for a locally free sheaf $\mathcal{F}$ and $\mathcal{M} \in \mathrm{D}^{b}\left[\bmod ^{c}(\widetilde{\mathcal{D}})\right]$ we have $\mathbb{D}_{D}(\mathcal{F} \otimes \mathcal{M}) \cong \mathcal{F}^{*} \otimes \mathbb{D}_{D}(\mathcal{M})$ canonically. Extending this isomorphism to bounded complexes of locally free coherent sheaves we get a canonical isomorphism

$$
\mathbb{D}_{D}(\mathcal{F} \otimes \mathcal{M}) \cong \mathbb{D}_{\mathcal{O}}(\mathcal{F}) \otimes \mathbb{D}_{D}(\mathcal{M})[-\operatorname{dim} \mathfrak{g}]
$$

We restrict attention to the formal neighborhood of $\mathcal{B}_{\chi} \times\{\nu\}$ and plug in $\mathcal{M}=\mathcal{M}_{\chi}^{-\rho+\nu}$. The desired isomorphism follows then from Lemma 3.0.6.b.

We are ready to prove

Proposition 3.0.9. Let $\nu \in \Lambda$ be regular and $\chi \in \mathfrak{g}^{*(1)}$ be nilpotent. Then for $\mathcal{F}$ in $\mathrm{D}^{b}\left[\mathcal{C o h}_{\mathcal{B}_{\chi}}\left(\tilde{\mathfrak{g}}^{*}\right)\right]$ we have a canonical isomorphism

$$
\left[\gamma_{\chi,-\rho+\nu}(\mathcal{F})\right]^{\sim} \cong \gamma_{-\chi,-\rho-\nu}\left(\sigma^{*} \mathbb{D}_{\mathcal{O}}(\mathcal{F})\right)
$$

Proof. Compare Lemma 3.0.3 with Corollary 3.0.8 and Lemma 3.0.1.

3.0.10. In the assumptions of the Proposition, assume moreover that $\nu \in \Lambda$ lies in the fundamental alcove $A_{0}$. Recall that for such $\nu$ there is an isomorphism isom $_{\nu}: B_{\text {aff }}^{\prime} \rightarrow{ }^{\Theta} B_{\text {aff }}^{\prime}$, where $\Theta$ is the $W_{\text {aff }}^{\prime}$ orbit of $\nu$. Denote $\mathbf{w}_{0} \stackrel{\text { def }}{=}$ isom $_{\nu}\left(w_{0}\right)$. Let $\nu^{\llcorner}=-w_{0}(\nu)$ be the dual weight.

Corollary 3.0.11. For $\mathcal{F}$ in $\mathrm{D}^{b}\left[\mathcal{C}_{\text {oh }} \mathcal{B}_{\chi}(1)\left(\widetilde{\mathfrak{g}}^{*}(1)\right)\right]$ we have a canonical isomorphism

$$
\left[\gamma_{\chi, \nu}(\mathcal{F})\right]^{\sim} \cong \mathbf{I}_{\widetilde{\mathbf{w}}_{0}}^{-1} \circ \gamma_{-\chi, \nu} \circ \mathbb{D}_{\mathcal{O}} \circ \sigma^{*}(\mathcal{F})
$$


Proof. In view of the last proposition we have

$$
\left[\gamma_{\chi, \nu}(\mathcal{F})\right]^{\sim} \cong \gamma_{-\chi,-\nu-2 \rho}\left(\sigma^{*} \mathbb{D}_{\mathcal{O}}(\mathcal{F})\right)
$$

We have $w_{0} \bullet(-\nu-2 \rho)=\nu^{\iota}=(-\nu-2 \rho) * \mathbf{w}_{0}$. It is clear that $\mathbf{w}_{0}$ increases the weight $-\nu-2 \rho$. Thus Theorem 2.1.4 and the definition of $\gamma_{\chi, \nu}$ show that

$$
\gamma_{-\chi, \nu^{\llcorner}} \cong \mathbf{I}_{\mathbf{W}_{0}} \circ \gamma_{-\chi,-\nu-2 \rho}
$$

The two displayed isomorphisms yield the result.

Remark 3.0.12. This description of duality on the category of modules (especially the particular case $\nu=\nu^{\swarrow}=0$ ) should be compared to the definition of a certain involution on the $K$-group of a Springer fiber in [Lu, Part II]. This remark will be elaborated in a future paper, where we plan to use the last corollary to show that Lusztig's involution preserves the classes of irreducible modules (lifted in a certain canonical way to the equivariant $K$-group); cf. also [B, 2.13, 2.17].

\section{REFERENCES}

[BB1] A. Beilinson and A. Bernstein, Localisation de g-modules, C. R. Acad. Sci. Paris Sér. I Math., 292 (1981), 15-18.

[BB2] A. Beilinson and A. Bernstein, A generalization of Casselman's submodule theorem, Representation theory of reductive groups (Park City, Utah, 1982), Progr. Math. 40, Birkhäuser, Boston (1983), pp. 35-52.

[BeG] A. Beilinson and V. Ginzburg, Wall-crossing functors and D-modules, Represent. Theory, 3 (1999), 1-31.

[B] R. Bezrukavnikov, Noncommutative counterparts of the Springer resolution, Proceeding of the International Congress of Mathematicians, Madrid, Spain, 2006, vol. 2, pp. 1119-1144.

[BrBr] R. Bezrukavnikov and A. Braverman, Geometric Langlands correspondence for $D$-modules in prime characteristic: the $G L(n)$ case, Quartely J. Pure Appl. Math., to appear.

[BMR] R. Bezrukavnikov, I. Mirković and D. Rumynin, Localization of modules for a semisimple Lie algebra in prime characteristic, with an appendix by S. Riche, preprint math.RT/0205144, to appear in Ann. Math.

[BK] A. Bondal and M. Kapranov, Representable functors, Serre functors, and mutations, Izv. Ak. Nauk, 53 (1989), 1183-1205; translation in Math. USSR-Izv., 35 (1990), 519-541.

[Bo] A. Borel et. al., Algebraic D-modules, Perspectives in Mathematics, 2, Academic Press, Boston, 1987. 
[BKR] T. Bridgeland, A. King and M. Reid, The McKay correspondence as an equivalence of derived categories, J. Amer. Math. Soc., 14 (2001), no. 3, 535-554 (preprint version Mukai implies McKay available as math.AG/9908027 at xxx.lanl.gov).

$[\mathrm{BrKu}] \mathrm{M}$. Brion and S. Kumar, Frobenius splitting methods in geometry and representation theory, Progress Math. 231, Birkhäuser, Boston, 2005.

[Bro] B. Broer, Normality of some nilpotent varieties and cohomology of line bundles on the cotangent bundle of the flag variety, Lie Theory and Geometry, Progress Math. 123, Birkhäuser, Boston (1994), pp. 1-19.

[BrGo] K. Brown and K. Goodearl, Homological aspects of Noetherian PI Hopf algebras of irreducible modules and maximal dimension, J. Algebra, 198 (1997), 240-265.

[BG] K. Brown and I. Gordon, The ramification of centres: Lie algebras in positive characteristic and quantized enveloping algebras, Math. Z., 238 (2001), 733-779.

[CG] N. Chriss and V. Ginzburg, Representation theory and complex geometry, Birkhäuser, Boston, 1997.

[De] M. Demazure, Invariants symétriques entiers des groupes de Weyl et torsion, Invent. Math., 21 (1973), 287-301.

[HKR] Y. Hashimoto, M. Kaneda and D. Rumynin, On localization of $\bar{D}$-modules, Representations of Algebraic Groups, Quantum Groups, and Lie Algebras, Contemp. Math. 413, AMS, Providence (2006), pp. 43-62.

[Ja] J. Jantzen, Representations of Lie algebras in prime characteristic, Representation theories and algebraic geometry, Proceedings NATO ASI (Montreal, 1997), Kluwer, Dordrecht (1998), pp. 185-235.

$[\mathrm{KW}]$ V. Kac and B. Weisfeiler, Coadjoint action of a semisimple algebraic group and the center of the enveloping algebra in characteristic p, Indag. Math., 38 (1976), 136-151.

[Lu] G. Lusztig, Bases in equivariant K-theory, Represent. Theory, 2 (1998), 298-369. Bases in equivariant K-theory II, Represent. Theory, 3 (1999), 281-353.

[Lu1] G. Lusztig, Hecke algebras and Jantzen's generic decomposition patterns, Adv. in Math., 37 (1980), 121-164.

[Mi] D. Miličić, Localization and Representation Theory of Reductive Lie Groups, available at http://www.math.utah.edu/ milicic.

[Mln] J. S. Milne, Etale cohomology, Princeton Math. Series 33, Princeton U. Press, 1980.

[MR] I. Mirković and D. Rumynin, Centers of reduced enveloping algebras, Math. Z., 231 (1999), 123-132.

[OV] A. Ogus and V. Vologodsky, Nonabelian Hodge theory in characteristic p, preprint math.AG/0507476.

[R] M. Reid, McKay correspondence, preprint alg-geom/9702016.

[Sk] A. Skorobogatov, Torsors and rational points, Cambridge U. Press, Cambridge, 2001.

[Ve] F. Veldkamp, The center of the universal enveloping algebra of a Lie algebra in characteristic p, Ann. Sci. École Norm. Sup. (4), 5 (1972), 217-240. 
Roman Bezrukavnikov

Department of Mathematics

Massachusetts Institute of Technology

77 Massachusetts ave.

Cambridge, MA 02139

U.S.A.

bezrukav@math.mit.edu

Ivan Mirković

Department of Mathematics and Statistics

University of Massachusetts

Amherst, MA 01003

U.S.A.

mirkovic@math.umass.edu

Dmitriy Rumynin

Mathematics Department

University of Warwick

Coventry, CV4 7AL

England

rumynin@maths. warwick.ac.uk 